Original paper

\title{
Metamorphic history of skarns, origin of their protolith and implications for genetic interpretation; an example from three units of the Bohemian Massif
}

\author{
Jaroslava PERTOLDOVÁ1*, Patricie TÝCOVÁ1 ${ }^{1}$ Kryštof VERNER ${ }^{1}$, Monika KOŠULIČOVÁ1, \\ Zdeněk PERTOLD², Jan KOŠLER³ , Jiř́ KONOPÁSEK' ${ }^{1}$ Marta PUDILOVÁ2
}

\author{
${ }^{1}$ Czech Geological Survey, Klárov 3, 11821 Prague 1, Czech Republic; jaroslava.pertoldova@geology.cz \\ ${ }^{2}$ Institute of Geochemistry, Mineralogy and Mineral Resources, Charles University, Albertov 6, Prague 2, 128 43, Czech Republic \\ ${ }^{3}$ Centre for Geobiology and Department of Earth Science, University of Bergen, Allegaten 41, N-5007 Bergen, Norway \\ ${ }^{*}$ Corresponding author
}

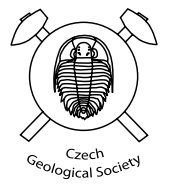

\begin{abstract}
Skarns in the Svratka Unit, in the neighbouring part of the Moldanubian Zone and in the Kutna Hora Complex were studied with respect to their metamorphic evolution, major- and trace-element geochemistry, oxygen isotopic composition and zircon ages. Skarns form competent lenses and layers in metamorphosed siliciclastic rocks and preserve some early deformation structures and several equilibrium assemblages representing the products of successive metamorphic reactions. The main rock-forming minerals, garnet and clinopyroxene, are accompanied by less abundant magnetite, amphibole, plagioclase, epidote \pm quartz. In the Svratka Unit the early prograde $\mathrm{M}_{1}$, prograde/peak $\mathrm{M}_{2}$, and retrograde $\mathrm{M}_{3}$ metamorphic stages have been distinguished. Metamorphic conditions in skarns of the Moldanubian Zone are limited to a relatively narrow interval of amphibolite facies. The prograde and retrograde events in the Kutná Hora Complex skarns probably took place under amphibolite-facies conditions. The presence of magnetite and the increasing proportion of the andradite component in the garnet indicate locally increased oxygen fugacity.

Skarn geochemistry does not show systematic differences in the skarn composition among the three units. The regional variations are exceeded by differences among samples from individual localities. The $\mathrm{Al}_{2} \mathrm{O}_{3} / \mathrm{TiO}_{2}, \mathrm{Al}_{2} \mathrm{O}_{3} / \mathrm{Zr}, \mathrm{TiO}_{2} / \mathrm{Nb}$ ratios point to the variable proportion of the detrital material, combined in skarn protoliths with $\mathrm{CaO}$ and $\mathrm{FeO}$, the major non-detrital components. The skarns exhibit elevated abundances of $\mathrm{Cu}, \mathrm{Zn}, \mathrm{Sn}$ and $\mathrm{As}$. The $\mathrm{Eu} / \mathrm{Eu} *$ ratio varies in the range of $0.5-8.6$, the total REE contents vary from 8 to $345 \mathrm{ppm}$. The lowest $\Sigma$ REE values $(<100 \mathrm{ppm})$ occur in skarns with magnetite mineralization. The wide intervals of $\Sigma \mathrm{REE}$ and $\mathrm{Eu} / \mathrm{Eu}^{*}$ values are interpreted to indicate variations in the temperature and redox conditions among layers of the same locality and at various localities.

The oxygen isotope compositions of garnets, pyroxenes and amphiboles from skarns of the Svratka Unit exhibit a range of $\delta^{18} \mathrm{O}=0.1$ to $4.1 \%$. In situ (laser-ablation ICP-MS) U-Pb dating of zircon from one of the Svratka Unit skarn bodies yielded a wide range of ages $(0.5-2.6 \mathrm{Ga})$, supporting the detrital origin of this zircon population.

The skarn protoliths were probably rocks of mixed detrital-exhalative origin deposited on the sea floor.

The geological position of skarns, with their structural and metamorphic record, probably reflect tectono-metamorphic evolution shared with that of their host rocks. The geochemical characteristics, including oxygen isotopic compositions and the presence of detrital zircons with a wide range of ages exclude metasomatic, and point to a sedimentary-exhalative mode of origin for the studied skarns.
\end{abstract}

Keywords: skarn, Bohemian Massif, petrology, geochemistry, oxygen isotopes, detrital zircon age

Received: 1 April 2009; accepted 11 June 2009; handling editor: W. S. Faryad

The online version of this article (http://dx.doi.org/10.3190/jgeosci.044) contains supplementary electronic material.

\section{Introduction}

Detailed modern contributions dealing with the nature and genesis of skarns were published by many geologists during the past few decades from different geological environments all over the world, for instance Jamtveit et al. (1993), Nicolescu et al. (1998), Meinert et al. (2003) or Gaspar et al. (2008).

The review of world skarn deposits has been presented by Einaudi et al. (1981), Burt (1982), Meinert (1998) and
Meinert et al. (2005). These publications and reviews are devoted to skarns with mostly contact metamorphic and metasomatic history accompanied by igneous-related hydrothermal input. Several authors have studied the mineralogy/petrology and genetic aspects of various skarn bodies in the Bohemian Massif, partly with emphasis on the pre-metamorphic history and open- $v s$. closed-system conditions of formation.

Formation under the open-system conditions implies a metasomatic mode of formation (i.e., metasomatism 
of carbonate rocks by granite-related fluids). This category includes several skarns in the Moldanubian Zone (Koutek 1950; Němec 1991; Houzar and Šrein 1995; Litochleb et al. 1997; Žáček et al. 1997, 2003), in the Krušné hory/Erzgebirge area (Němec 1991; Šrein and Šreinová 2000). Rötzler and Mingram (1998) reported a skarn formation via Ca-metasomatism of basic magmatic rocks (rodingites).

Skarn formation in a closed system, due to regional metamorphism of protoliths with suitable composition, was assumed in the Moldanubian Zone by Zoubek (1946), Vrána (1987), Potužák (1996), Pertold et al. (1997, 2000), Drahota et al. (2005) and in the Krušné hory/Erzgebirge area by Klomínský and Sattran (1963) with Kotková (1991), in the Lugicum by Pertold and Pouba (1982), and in the Svratka Unit by Pertoldová (1986) and Pertoldová et al. (1998).

The aim of this paper is to address the widely discussed problem of the origin of the Bohemian skarns and their pre-metamorphic precursors. The study includes skarns from the Varied Group, Monotonous Group and the Gföhl Unit of the Moldanubian Zone, together with the Kutná Hora Complex and Svratka Unit. Altogether, 34 localities of skarn rocks were studied, with their basic characteristics and locations presented in Tab. 1. The paper deals with the geological position, metamorphic history and whole-rock geochemistry. The oxygen isotope composition of silicate minerals was studied in selected samples from the Svratka Unit. Zircon populations in skarn from the Svratouch locality were dated. The geological positions of the sampled skarn localities are depicted in Fig. 1. Using this integrated data set, it will be demonstrated that the studied skarns have formed by a closed-system metamorphism of sedimentary-exhalative rocks deposited on the sea floor.

\section{Geological setting}

The Variscan Orogeny (subduction of the "Tethys-type" oceanic crust followed by continental collision of Gondwana and Laurussia; 380-290 Ma ago) was of major importance for amalgamation and evolution of basement units in the Bohemian Massif (for a review, see Franke 1989, 2000; Tait et al. 2000). In the north-eastern part of the Bohemian Massif, this orogeny involved tectonometamorphic processes including juxtaposition and stacking of contrasting segments of the continental crust. At a somewhat later stage, the eastern parts of these amalgamated units were overthrust on Neoproterozoic crustal segment of the Brunovistulicum. On a regional scale, the studied area includes two super-units (Fig. 1) -

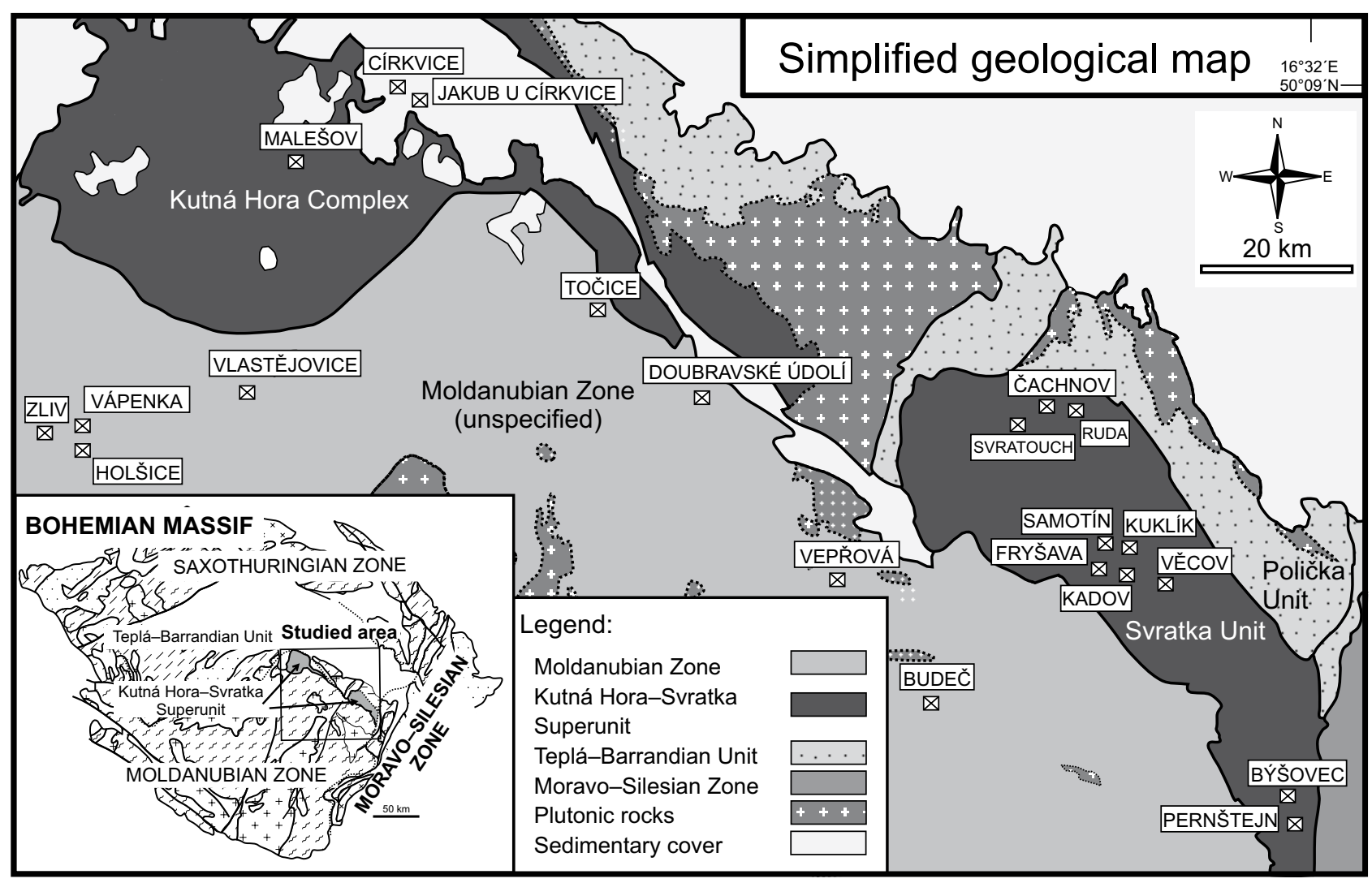

Fig. 1 Simplified geological map of the NE margin of the Moldanubian Zone, showing skarn localities. Modified after Cháb et al. (2007). Inset: Sketch of Bohemian massif modified after Franke et al. (2000). 
Tab. 1 List of analyzed skarn samples from the studied units

\begin{tabular}{|c|c|c|c|c|c|c|c|c|c|c|}
\hline $\begin{array}{c}\text { Geological } \\
\text { setting }\end{array}$ & $\begin{array}{c}\text { Sample } \\
\text { No. }\end{array}$ & Sample & Locality & $\begin{array}{l}\text { Prevailing } \\
\text { mineralogy }\end{array}$ & $\begin{array}{c}\text { Microprobe } \\
\text { analysis }\end{array}$ & $\begin{array}{c}\text { WR geochem. } \\
\text { analysis }\end{array}$ & Dating & $\begin{array}{c}\text { Oxygen } \\
\text { analysis }\end{array}$ & WGS-84-N & WGS-84-E \\
\hline \multirow{27}{*}{ 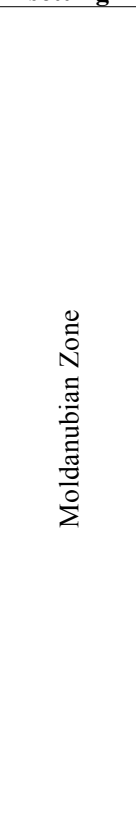 } & 1 & KM8A & Budeč & Grt, Cpx & $\times$ & $\times$ & & & 4932.25 & 1554.97 \\
\hline & 2 & KM8B & Budeč & Mag, Cpx, Grt & $x$ & $x$ & & & 4932.25 & 1554.97 \\
\hline & 3 & KM8C & Budeč & Grt, Cpx & $\times$ & & & & 4932.25 & 1554.97 \\
\hline & 4 & KM8FV1 & Budeč & Mag, Cpx, Grt & & $x$ & & & 4932.25 & 1554.97 \\
\hline & 5 & KM8FV2 & Budeč & $\mathrm{Cpx}$ & & $x$ & & & 4932.25 & 1554.97 \\
\hline & 6 & KM3F & Vepřová & Grt, Cpx & & $\times$ & & & 4937.86 & 1550.18 \\
\hline & 7 & KM3H & Vepřová & Qtz, Cpx & & $x$ & & & 4937.86 & 1550.18 \\
\hline & 8 & $\mathrm{KM} 3 \mathrm{CH}$ & Vepřová & Grt, Cpx & & $x$ & & & 4937.86 & 1550.18 \\
\hline & 9 & KM3A & Vepřová & Grt, Cpx & $\times$ & & & & 4937.86 & 1550.18 \\
\hline & 10 & KM3C & Vepřová & Grt, Cpx & $x$ & & & & 4937.86 & 1550.18 \\
\hline & 11 & KM3E & Vepřová & Grt, Cpx & $x$ & & & & 4937.86 & 1550.18 \\
\hline & 12 & KM3M & Vepřová & Grt, Cpx & $x$ & & & & 4937.86 & 1550.18 \\
\hline & 13 & KM3FV & Vepřová & Grt, Cpx & & $x$ & & & 4902.45 & 1609.16 \\
\hline & 14 & KMRES1 & Rešice & Grt, Cpx & & $x$ & & & 4902.45 & 1609.16 \\
\hline & 15 & KMRES2 & Rešice & Cpx, Grt & & $x$ & & & 4902.45 & 1609.16 \\
\hline & 16 & KMRES3 & Rešice & Grt, Cpx & & $x$ & & & 4902.45 & 1609.16 \\
\hline & 17 & KMRES5 & Rešice & Cpx & & $x$ & & & 4902.45 & 1609.16 \\
\hline & 18 & KMSLA1 & Slatina & Grt & & $x$ & & & 4901.25 & 1601.31 \\
\hline & 19 & KMSLA2 & Slatina & Cpx, Grt & & $x$ & & & 4901.25 & 1601.31 \\
\hline & 20 & KMVLAFV1 & Vlastějovice & Grt, Cpx & & $x$ & & & 4944.26 & 1511.09 \\
\hline & 21 & KMVLAFV2 & Vlastějovice & Cpx, Grt, Pl & & $x$ & & & 4944.26 & 1511.09 \\
\hline & 22 & KMHOLFV1 & Holšice & Grt, Cpx & & $x$ & & & 4945.58 & 1503.03 \\
\hline & 23 & KMZLIFV1 & Zliv & $\mathrm{Cpx}$ & & $x$ & & & 4946.01 & 1502.34 \\
\hline & 24 & KMZLIFV2 & Zliv & Cpx, Grt & & $x$ & & & 4946.01 & 1502.34 \\
\hline & 25 & KMVAPFV2 & Vápenka & Grt, $\mathrm{Hbl}$ & & $x$ & & & 4946.11 & 1502.41 \\
\hline & 26 & KMZUPFV1 & Županovice & Grt, Cpx & & $x$ & & & 4856.59 & 1530.06 \\
\hline & 27 & KMZUPFV2 & Županovice & Cpx & & $\times$ & & & 4856.59 & 1530.06 \\
\hline \multirow{30}{*}{ 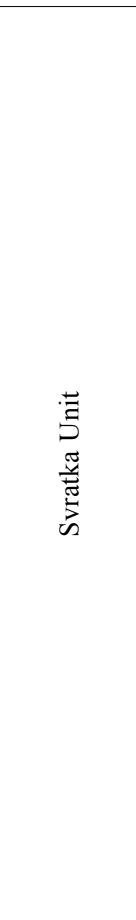 } & 28 & KM5d & Fryšava & Grt, Hbl & & $x$ & & & 4937.70 & 1602.77 \\
\hline & 29 & KM5A & Fryšava & Grt, Cpx & & $x$ & & & 4937.70 & 1602.77 \\
\hline & 30 & KM6A & Kadov & Grt, Cpx & & $\times$ & & & 4939.68 & 1605.15 \\
\hline & 31 & SV1 & Svratouch & Grt, Cpx & $x$ & & & & 4944.32 & 1601.66 \\
\hline & 32 & SV27 & Svratouch & $\mathrm{Cpx}, \mathrm{Grt}$ & & $x$ & & & 4944.32 & 1601.66 \\
\hline & 33 & SV262 & Svratouch & Cpx, Grt, Gru & $x$ & & $x$ & $x$ & 4944.32 & 1601.66 \\
\hline & 34 & SV25 & Svratouch & $\mathrm{Cpx}, \mathrm{Grt}$ & $x$ & $x$ & $x$ & $x$ & 4944.32 & 1601.66 \\
\hline & 35 & MSDC114 & Teplá & Grt, Cpx & & $\times$ & & $x$ & 4938.66 & 1605.09 \\
\hline & 36 & MSDC 130 & Blatiny & Grt, Cpx & & $x$ & & $x$ & 4938.93 & 1605.32 \\
\hline & 37 & MSDC 128 & Samotín & Grt, Cpx & & $x$ & & $x$ & 4938.83 & 1604.91 \\
\hline & 38 & KMSB6 & Adamova skála & Cpx, Grt & & $x$ & & & 4937.38 & 1609.20 \\
\hline & 39 & KMSB7 & Malá skála & $\mathrm{Grt}, \mathrm{Cpx}$ & & $x$ & & & 4937.45 & 1608.47 \\
\hline & 40 & KMSB15 & Líšná & Cpx, Grt & & $x$ & & & 4938.44 & 1608.41 \\
\hline & 41 & KMKUKFV1 & Kuklík & $\mathrm{Cpx}, \mathrm{Grt}$ & & $x$ & & & 4937.80 & 1606.81 \\
\hline & 42 & KMKUKFV2 & Kuklík & Cpx & & $x$ & & & 4937.80 & 1606.81 \\
\hline & 43 & KMKUKFV3 & Kuklík & $\mathrm{Cpx}$ & & $x$ & & & 4937.80 & 1606.81 \\
\hline & 44 & KM7 & Kuklík & Grt, Cpx & $x$ & $x$ & & & 4938.14 & 1606.20 \\
\hline & 45 & KM7FV & Krátká & Grt, Cpx & & $x$ & & & 4938.14 & 1606.20 \\
\hline & 46 & KMSB21 & Věcov & Grt, Cpx & $x$ & & & & 4937.12 & 1609.30 \\
\hline & 47 & KMLISFV & Líšná & Grt, Cpx & & $x$ & & & 4938.44 & 1608.41 \\
\hline & 48 & VR1 & Čachnov & Cpx, Grt & & $x$ & & & 4945.09 & 1602.85 \\
\hline & 49 & Cach2 & Čachnov & Grt & $x$ & $x$ & & & 4945.09 & 1602.85 \\
\hline & 50 & VR10B & Čachnov & Grt, Hbl, Cpx & & $x$ & & & 4945.09 & 1602.85 \\
\hline & 51 & VR39 & Ruda u Čachnova & $\mathrm{Grt}, \mathrm{Hbl}$ & & $x$ & & & 4944.55 & 1604.69 \\
\hline & 52 & VR60 & Ruda u Čachnova & Grt, $\mathrm{Hbl}$ & & $x$ & & & 4944.55 & 1604.69 \\
\hline & 53 & VR69 & Ruda u Čachnova & Grt, Cpx & & $x$ & & & 4944.55 & 1604.69 \\
\hline & 54 & KM11 & Býšovec & Grt, Cpx & & $x$ & & & 4928.43 & 1617.67 \\
\hline & 55 & KMPERFV1 & Pernštejn & Grt & & $x$ & & & 4926.98 & 1618.33 \\
\hline & 56 & KMPERFV2 & Pernštejn & Grt & & $x$ & & & 4926.98 & 1618.33 \\
\hline & 57 & KMPERFV3 & Pernštejn & Grt, Cpx & & $\times$ & & & 4926.98 & 1618.33 \\
\hline \multirow{12}{*}{ 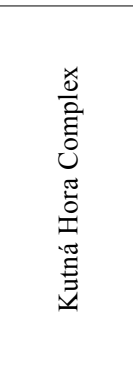 } & 58 & KMMALFV1 & Malešov & Cpx, Grt, Mag & & $x$ & & & 4955.52 & 1513.56 \\
\hline & 59 & KMMALFV2 & Malešov & Grt, Cpx, Mag & & $x$ & & & 4955.52 & 1513.56 \\
\hline & 60 & KMVSM1 & Malešov & Cpx, Grt & $x$ & $x$ & & & 4955.52 & 1513.56 \\
\hline & 61 & KMVSM2 & Malešov & Grt, Cpx & $x$ & $x$ & & & 4955.52 & 1513.56 \\
\hline & 62 & KMVSM4 & Malešov & Grt, Cpx & $x$ & & & & 4955.52 & 1513.56 \\
\hline & 63 & KMVSM5 & Malešov & $\mathrm{Cpx}, \mathrm{Grt}, \mathrm{Hbl}$ & $x$ & & & & 4955.52 & 1513.56 \\
\hline & 64 & KMVSM7 & Malešov & Grt, Cpx, Ep & $x$ & & & & 4955.52 & 1513.56 \\
\hline & 65 & KMCIRFV1 & Církvice & Grt, Cpx & & $\times$ & & & 4956.83 & 1520.32 \\
\hline & 66 & KMCIRFV2 & Církvice & Grt, Cpx & & $x$ & & & 4956.83 & 1520.32 \\
\hline & 67 & KMV20 & Jakub u Církvice & Grt, Cpx & $x$ & $x$ & & & 4957.16 & 1520.29 \\
\hline & 68 & KMTOCFV & Točice & Grt, Cpx & & $\times$ & & & 4948.15 & 1533.83 \\
\hline & 69 & BV1 & Točice & Grt, Cpx & & $x$ & & & 4948.15 & 1533.83 \\
\hline
\end{tabular}

WGS-84 = World Geodetic System 1984 
the Moldanubian Zone (MZ) and the Kutná Hora-Svratka Superunit (KHS). A detailed review of the geology and structure of the basement units along the north-eastern margin of the Moldanubian Zone is presented by Verner et al. (this volume).

\subsection{Svratka Unit}

The Svratka Unit (SU) is composed of migmatites, metagranites, orthogneisses and metasedimentary rocks with layers of calc-silicate gneisses and amphibolites. The Variscan tectonometamorphic conditions were estimated at $\sim 0.4-0.8 \mathrm{GPa}$ and $\sim 650-670{ }^{\circ} \mathrm{C}$ (Pitra and Guiraud 1996; Buriánek 2008; Buriánek and Čopjaková 2008). The rocks of the SU have retained some relics of Cambro-Ordovician structures; the SU belongs to the mid-crustal level of the Variscan orogen (Schulmann et al. 2005; Verner et al. this volume).

The dominant planar fabric in this unit is NW-SE trending, except for the western termination, which is a large-scale antiformal closure. Foliation planes with predominating moderate dips contain subhorizontal lineation defined by elongated mineral aggregates (Fig. 2a). Relics of older deformation structures are locally preserved in more competent rocks, particularly in calc-silicate gneisses and skarns. The skarn bodies are moderately to strongly elongated, with dimensions of $\sim 0.1-0.3 \mathrm{~km}$ $\times 0.5-1.5 \mathrm{~km}$, often oriented parallel with the structures in the host rocks.

The dominating structure in skarns is compositional layering, usually consisting in alternating garnet-rich and pyroxene-rich bands. This planar fabric is typically oriented parallel with the foliation in the enclosing rocks. Relics of older structures (isoclinal and rootless folds) show steeply dipping foliation trending WNW-ESE, especially in limbs of asymmetric folds (Fig. 2a). Minor structural elements include superimposed anastomosing cleavage domains and shear zones. The geological positions of skarn bodies in SU are documented in 1:25000 maps (Hanžl ed. 2008; Melichar ed. 2008).

\subsection{North-eastern part of the Moldanubian Zone}

The Moldanubian Zone (MZ) represents exhumed lowerto mid-crustal rocks that recorded a polyphase Variscan tectonometamorphic history (for a general review see Urban and Synek 1995; Schulmann et al. 2005, 2009). The structural development was related to stacking of individual lithotectonic units at $\sim 350-340 \mathrm{Ma}$, followed by HT-LP metamorphism and anatexis at $\sim 340-330 \mathrm{Ma}$ and subsequent (Late Carboniferous to Permian) wrench tectonics (Edel et al. 2003). The northern and northeastern parts of the MZ, locally termed the Strážek Unit, include migmatized paragneisses and migmatites with abundant layers of amphibolite, calc-silicate gneiss, quartzite and relatively small bodies of HP/HT granulite and peridotite/lherzolite. The metamorphic conditions of granulites were estimated by Tajčmanová et al. (2006) at $\sim 1.8 \mathrm{GPa}$ and $\sim 850^{\circ} \mathrm{C}$ for the high-pressure event, followed by low-pressure re-equilibration at $0.4-0.6 \mathrm{GPa}$ and $680-720^{\circ} \mathrm{C}$.

The structural patterns in the northern and northeastern parts of the MZ and metamorphic conditions show notable variation due to complicated superposition of structures. Along the northern margin of the MZ, foliations are parallel with the border of the unit and exhibit a shallow to moderate dip to the north or northwest. Skarn bodies in the northern part of the MZ are hosted by migmatites and migmatized paragneisses. They form asymmetric bodies with longer axis of $\sim 50-500 \mathrm{~m}$ and internal structures that are usually concordant with the regional structures of the enclosing paragneisses (Drahota et al. 2005). The geological situation of the skarn bodies in the northern part of the MZ is presented in the Czech Geological Survey maps 1:25 000 (Hrdličková ed. 2008; Hanžl and Hrdličková eds 2009; Rejchrt ed. 2009).

\subsection{Kutná Hora Complex}

The Kutná Hora Complex (KHC) is a heterogeneous assemblage of lower- to mid- crustal segments at the northern border of the MZ, comprising orthogneisses, migmatites, metasediments and ultramafic upper mantle rocks (Synek and Oliveriová 1993). The metamorphic evolution of the upper part of the KHC (Běstvina, Malín and Plaňany Unit) corresponds to the HP/HT-HP/MT events (Vrána et al. 2005) followed by HP/MT tectonometamorphic overprint. The lower part of the $\mathrm{KHC}$ is composed of the Kouřim Nappe (fine-grained leucocratic migmatites and orthogneisses) and further, in the marginal part, of the Micaschist Unit (metapelites). Rocks of these two units recorded a Variscan MP-MT metamorphic event (Nahodilová et al. 2006).

The tectonic structure of the KHC is interpreted as resulting from polyphase deformation and the probable effects of shallow-dipping thrusting, similar to nappe structures. The early structures include $\mathrm{N}-\mathrm{S}$ trending foliation locally preserved in orthogneisses and migmatites. They are usually strongly overprinted by younger foliation with shallow to moderate dip to the NNW to ENE. Review of structural information is presented by Synek and Oliveriová (1993) and Nahodilová et al. (2005). The Malešov skarn body (Figs 1, 2b) is located in the structurally highest part of the KHC, the Malín 
Unit, composed of polymetamorphic migmatites and two-mica kyanite migmatites with boudin-like bodies of upper mantle garnet lherzolites. Metamorphic foliation in the rocks of the Malín Unit is folded to open, moderately asymmetric folds but it is generally dipping at moderate to low angles to $\sim \mathrm{NNW}$. The Malešov skarn body $(\sim 200 \times 1000 \mathrm{~m})$ forms an asymmetric antiformal structure with limbs dipping at moderate to low angles to WNW and NE (Fig. 2b). The internal structure of skarn is defined by alternating irregular bands rich in garnet or pyroxene. These planar structures dip steeply to the NNW to $\mathrm{W}$. They are thus oriented discordantly in relation to the metamorphic planar fabrics predominating in the enclosing rocks.

\section{Analytical methods}

Mineral analyses were carried out with a Cameca Camebax SX-100 in the Joint Laboratory of Electron Microscopy and Microanalysis of Masaryk University and the Czech Geological Survey in Brno. Operating conditions were $15 \mathrm{kV}$ accelerat-
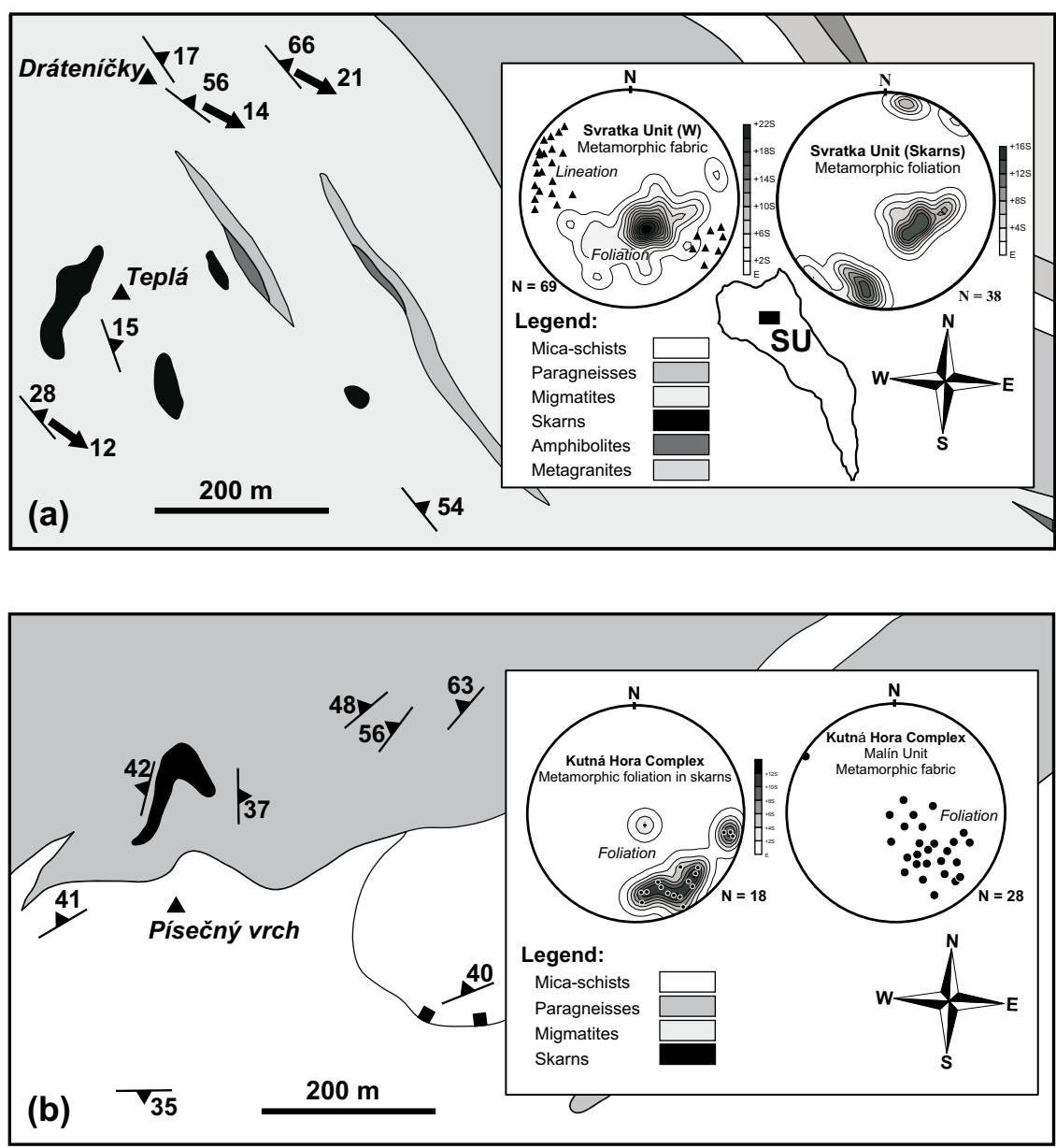

Fig. 2 Schematic structural maps of the two selected skarn bodies: a - Samotín (western part of the Svratka Unit); b - Malešov (SE part of the Kutná Hora Complex).

ing voltage and $30 \mathrm{nA}$ beam current.

The beam was focused to $3 \mu \mathrm{m}$; the peak counting times were 20 seconds. The analyses were recalculated to chemical formulae or end member proportions using the MinPet software v. 2.02 of Richard (1995).

Whole-rock major- and trace-element concentrations were determined at the Central Laboratories of the Czech Geological Survey (CGS), Prague, or Acme Analytical Laboratories, Ltd., Vancouver. Major oxides were determined by FAAS, ICP-OES or titration methods (ČGS) and the ICP-MS method (Acme). The REE and other trace elements were analyzed by the ICP-MS, ICP-OES and XRF methods. Geochemical data were processed using the GCDkit software package (Janoušek et al. 2006).

The calculations of P-T conditions for mica schists were performed using Thermocalc 3.25 (Powell et al. 1998; 2005 upgrade) and the internally consistent thermodynamic dataset 5.5 (Holland and Powell 1998; November 2003 upgrade). Mixing models for most solid solutions were taken from White et al. (2001) and the THERMOCAlC documentation (Powell and Holland 2004).
Plagioclase was formulated using the model of Holland and Powell (2003), the paragonite-muscovite solution is after Coggon and Holland (2002) and the paragonitemargarite solution as in Štípská et al. (2006).

Oxygen for isotopic analyses was liberated from mineral separates by a fluorination technique using $\mathrm{BrF}_{5}$, similar to that of Clayton and Mayeda (1963), at the Institute of Geochemistry, Mineralogy and Mineral Resources, Charles University in Prague. During this procedure, oxygen is cryogenically cleaned to remove the reaction products and excess fluorination agent; finally it is converted to $\mathrm{CO}_{2}$ on a heated graphite rod. Isotopic measurements were performed using the Finnigan MAT 251 mass spectrometer at the Czech Geological Survey, Prague. The overall analytical uncertainty of the $\delta^{18} \mathrm{O}$ values tested by repeated analyses of NBS 28 standard was $0.2 \%$ (SMOW).

Zircon grains were extracted from rock samples using conventional crushing, heavy liquids and magnetic separation. The grains were mounted in 1 inch epoxy-filled blocks and polished to obtain even surfaces suitable for 
laser ablation ICP-MS analyses. Prior to analysis, the carbon coating was removed and the sample surfaces were cleaned in deionised water and ethanol. Isotopic analysis of zircons by laser ablation ICP-MS followed the technique described in Košler et al. (2002) with Košler and Sylvester (2003). A Thermo-Finnigan Element 2 sector field ICP-MS coupled to a 213 NdYAG laser (New Wave UP-213) at Bergen University was used to measure the $\mathrm{Pb} / \mathrm{U}$ and $\mathrm{Pb}$ isotopic ratios in zircons. The raw data were corrected for the dead time of the electron multiplier, processed off line in the Lamdate spreadsheet-based program (Košler et al. 2002) and plotted on concordia diagrams using Isoplot (Ludwig 1999). Data reduction included correction for the gas blank, laser-induced elemental fractionation of $\mathrm{Pb}$ and $\mathrm{U}$ and instrument mass bias. No common $\mathrm{Pb}$ correction was applied to the data. Zircon reference materials 91500 (1065 Ma - Wiedenbeck et al. 1995) and Plešovice (338 Ma - Sláma et al. 2008) were periodically analysed during this study.

\section{Petrography, mineral chemistry and metamorphic evolution}

Petrological description was done for the following samples: the Svratka Unit - SV1, SV262, SV25, KM7, KMSB21, Cach2; the Moldanubian Zone - KM8A, KM8B, KM8C, KM3A, KM3C, KM3E, KM3M, the Kutná Hora Complex - KMVSM1-7, KMV20.

The summary of petrological results is presented in Tab. 2.

\subsection{Svratka Unit}

Skarns exhibit banded or massive structure with nematoblastic or nemato-granoblastic texture.

The following skarn types were recognised under the microscope: garnet-pyroxene skarn, garnet-amphibole skarn, pyroxene skarn with garnet, grunerite-pyroxene skarn with garnet, and pyroxene-garnet skarn grading to

Tab. 2 The summary of petrological results for skarns from the Svratka Unit, the Moldanubian Zone and the Kutná Hora Complex

\begin{tabular}{|c|c|c|c|c|c|c|c|c|}
\hline \multirow{2}{*}{ unit } & \multirow{2}{*}{ skarn type } & \multirow{2}{*}{ texture } & \multirow{2}{*}{ structure } & \multicolumn{4}{|c|}{ minerals } & \multirow{2}{*}{ note } \\
\hline & & & & rock-forming & minor & acces. & secondary & \\
\hline 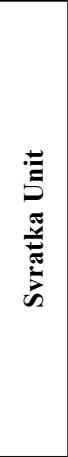 & $\begin{array}{c}\text { Grt-Cpx, Cpx-Grt, } \\
\text { garnerite, Cpx } \\
\text { skarn, Grt-Hbl, } \\
\text { Gru-Cpx }\end{array}$ & $\begin{array}{c}\text { nematoblastic, } \\
\text { nematogranoblastic }\end{array}$ & $\begin{array}{l}\text { banded, } \\
\text { massive }\end{array}$ & $\begin{array}{c}\operatorname{Grt}\left(\mathrm{Grs}_{21-44}\right. \\
\operatorname{Alm}_{25-78} \mathrm{Sps}_{0-23} \\
\left.\operatorname{Adr}_{0-12} \operatorname{Prp}_{0.5-7}\right), \mathrm{Cpx}\end{array}$ & $\begin{array}{l}\text { Qtz, Pl, Ep, } \\
\text { Mag }\end{array}$ & $\begin{array}{c}\text { Zrn, Spn, } \\
\text { Aln, Ap, } \\
\text { Ilm, Ccp, } \\
\mathrm{Au}, \mathrm{Py} \\
\text { native Bi }\end{array}$ & $\mathrm{Hbl}, \mathrm{Pl}$ & $\begin{array}{l}\text { Garnets show prograde } \\
\text { compositional zon- } \\
\text { ing. Decompression } \\
\text { textures: Spn } \rightarrow \text { Spn-P1 } \\
\text { symplectite, Grt } \rightarrow \text { Hbl- } \\
\text { Pl symplectite, } \\
\text { Cpx } \rightarrow \text { Cpx-Pl sym- } \\
\text { plectite. The content } \\
\text { of jadeite component } \\
\text { in Cpx is } 0.5-24 \\
\text { mol.\%. }\end{array}$ \\
\hline 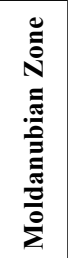 & $\begin{array}{c}\text { Grt-Cpx, Cpx-Grt, } \\
\text { garnerite, Cpx, } \\
\text { Mag with } \mathrm{Hbl}\end{array}$ & $\begin{array}{c}\text { nematoblastic, } \\
\text { nematogranoblastic }\end{array}$ & $\begin{array}{l}\text { banded, } \\
\text { massive }\end{array}$ & $\begin{array}{c}\text { older Grt }\left(\mathrm{Grs}_{74-67}\right. \\
\left.\text { Alm }_{9-12} \text { Adr }_{15-18}\right) \\
\text { Cpx, younger Grt } \\
\left(\text { Grs }_{34-35} \text { Alm }_{6-8} \text { Sps }_{4}\right. \\
\left.\text { Adr }_{53-54}\right)\end{array}$ & $\begin{array}{l}\text { Qtz, Pl, Ep, } \\
\text { Mag, Czo }\end{array}$ & $\begin{array}{c}\mathrm{Spn}, \mathrm{Ap}, \\
\text { native } \mathrm{Bi}, \\
\mathrm{Py}, \mathrm{Po}\end{array}$ & $\mathrm{Hbl}, \mathrm{Pl}$ & $\begin{array}{l}\text { Garnets show weak } \\
\text { retrograde zoning. } \\
\text { Younger Grt rich in } \\
\text { Adr fills fractures in } \\
\text { older Grt. }\end{array}$ \\
\hline 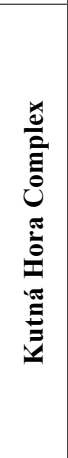 & $\begin{array}{c}\text { Grt-Cpx, Cpx-Grt, } \\
\text { garnerite, Cpx } \\
\text { with Grt }\end{array}$ & $\begin{array}{c}\text { nematoblastic, } \\
\text { nematogranoblastic }\end{array}$ & $\begin{array}{c}\text { banded, } \\
\text { massive, } \\
\text { schlieren-like }\end{array}$ & $\begin{array}{c}\text { Grt }\left(\mathbf{G r s}_{50-75} \operatorname{Alm}_{24-15}\right. \\
\left.\operatorname{Sps}_{2-0} \operatorname{Adr}_{31-9} \operatorname{Prp}_{1-3}\right), \\
\operatorname{Grt}_{\left(\mathbf{G r s}_{24-65} \operatorname{Alm}_{9-0}\right.} \\
\left.\text { Sps }_{4-0} \operatorname{Adr}_{65-33}\right), \mathbf{C p x}, \\
\text { Ep }\end{array}$ & Mag, Pl & \begin{tabular}{|} 
Spn, \\
Aln, Ap, \\
cerite, \\
hydro \\
grossular
\end{tabular} & $\begin{array}{c}\text { Grt, Ep, } \\
\text { Hbl }\end{array}$ & $\begin{array}{l}\text { Two types of garnet. } \\
\text { Both are characterized } \\
\text { by inverse variation in } \\
\text { the Grs and And com- } \\
\text { ponents. Grt and Cpx } \\
\text { show weak prograde } \\
\text { zoning. Four genera- } \\
\text { tions of Ep and three } \\
\text { generations of Cpx. } \\
\text { Some Ep are enriched } \\
\text { in REE. }\end{array}$ \\
\hline
\end{tabular}

(acces. $=$ accessory) 
garnetite \pm quartz. Garnet bands are several $\mathrm{mm}$ to several dm wide and often exhibit irregular folding, swelling to elongated lenses, or pass to garnet clusters and schlieren. Subordinate minerals, accompanying garnet and clinopyroxene, are quartz, plagioclase, epidote, amphibole, and accessory zircon, titanite, allanite and apatite. Some specimens exhibit reaction textures typical of decompression of eclogites or mafic granulites, such as amphiboleplagioclase symplectite coronas around garnet (Fig. 3a), clinopyroxene replaced by clinopyroxene-plagioclase symplectite (Fig. 3b) and Al-rich titanite transformed to titanite-plagioclase symplectite (Fig. 3c).

The following opaque minerals were identified in skarns: magnetite and ilmenite, locally cobaltite, arsenopyrite, pyrrhotite, chalkopyrite, pyrite, rare marcasite, löllingite, safflorite, safflorite-löllingite, FeCo-sulfarsenide, bismuthinite, native bismuth, gold, Bi-tellurides, and galena. A detailed petrological study of skarn localities near Pernštejn (western Moravia) was published by Pertoldová et al. (1998).

\subsubsection{Garnet}

Garnet grains enclose clinopyroxene, epidote, oligoclase, and exceptionally quartz. In other samples, garnet is affected by alteration, eventually it is completely replaced by plagioclase, epidote, amphibole and carbonate. Garnet compositions correspond dominantly to the grossularalmandine series $\left(\mathrm{Grs}_{21-44} \mathrm{Alm}_{25-78} \mathrm{Sps}_{0-23} \mathrm{Adr}_{0-12} \operatorname{Prp}_{0.5-7}\right.$, $\mathrm{X}_{\mathrm{Fe}}=0.95-1.00 \mathrm{~mol}$. \%; Fig. 2a). A prominent compositional zoning is defined by variation in the spessartine, almandine and grossular components (Fig. 5a-b, Tab. 3).

In one particular sample (Cach2), garnet exhibits two distinct compositional zones seen as optical zoning. The darker part corresponds to the crystal core, while the outer zone has a lighter colour (Fig. 3d, Tab. 3) and contains a higher proportion of andradite. These garnets contain more grossular than the almandine component $\left(\mathrm{Grs}_{45-73}\right.$ $\mathrm{Alm}_{13-23}$; Fig. 4). Representative chemical analyses of garnets from the Svratka Unit are presented in Tab. 3.

\subsubsection{Clinopyroxene}

Clinopyroxene compositions correspond to the diopsidehedenbergite series (Fig. 6, Tab. 4). The content of the jadeite component is $0.5-24 \mathrm{~mol} . \%$. Clinopyroxenes with elevated Jd component (up to $24 \mathrm{~mol}$. \%) occur in the matrix of some samples in pyroxene-plagioclase symplectite, or form inclusions in garnet (Fig. 3b).

\subsubsection{Calcic amphibole}

Amphiboles were frequently formed by replacement of clinopyroxene or garnet, such as in amphibole- plagioclase symplectite. Some samples contain quartzamphibole veinlets or a plagioclase-amphibole mosaic, filling domains among large garnet and pyroxene crystals (Fig. 3a). The chemical composition of these amphiboles corresponds to ferro-pargasite, hastingsite and ferroactinolite (Tab. 5).

\subsubsection{Grunerite}

The fine-grained matrix consists of subhedral grains of light green clinopyroxene intergrown with paler, prismatic to acicular crystals of grunerite (Fig. 3e, Tab. 5).

\subsubsection{Plagioclase}

Plagioclase occurs as a minor component in small veinlets with amphibole among grains of garnet and clinopyroxene or it forms symplectite coronas around garnet (Fig. 3a). The chemical composition varies between albite and andesine $\left(\mathrm{An}_{2-42}\right.$, Tab. 5).

\subsubsection{Titanite}

Titanite occurs as a common accessory mineral in skarns. Sample KMSB21c contains grains of inhomogeneous titanite, in part unmixed to plagioclase-titanite symplectite (Fig. 3c). The older titanite I shows notable enrichment in $\mathrm{Al}$ and $\mathrm{F}$ ( 2 to 6 wt. $\% \mathrm{Al}_{2} \mathrm{O}_{3}, 0.7$ to 2 wt. $\% \mathrm{~F}$ ), while younger titanite II in symplectite contains $1-3$ wt. \% $\mathrm{Al}_{2} \mathrm{O}_{3}$ and $0-1$ wt. \% F (Tab. 7).

\subsubsection{Metamorphic development}

Garnets exhibit well-defined prograde compositional zoning (Fig. 5a-b). The chemical composition of garnet and variation in the jadeite component in pyroxenes indicate at least three metamorphic episodes, similar to those in the enclosing rocks (see discussion).

The prograde zoning in garnet is defined by a high content of $\mathrm{Mn}$ in the crystal cores, corresponding to prograde stage $M_{1}$. The following prograde stage $M_{2}$ is one of a high-pressure increase from amphibolite facies to eclogite facies over a temperature interval of c. $100^{\circ} \mathrm{C}$. Peak metamorphic conditions are characterized by pressure near $1.4 \mathrm{GPa}$ (Gasparik and Lindsley 1980, see Fig. 13), expressed by jadeite component up to $24 \mathrm{~mol}$. \%. The increased Jd content corresponds to equilibration at eclogite-facies conditions. The following stage $M_{3}$ is related to exhumation of the Complex and decompression of skarns back to amphibolite-facies conditions. Reaction textures, such as amphibole-plagioclase coronas around garnet (Fig. 3a), disintegration of Jd-rich pyroxene to clinopyroxene-plagioclase symplectite 


Metamorphic history and protolith of skarns, Bohemian Massif

Tab. 3 Representative chemical analyses of garnets from the Svratka Unit, the Moldanubian Zone and the Kutná Hora Complex

\begin{tabular}{|c|c|c|c|c|c|c|c|c|c|c|c|c|c|c|c|c|}
\hline \multirow{3}{*}{$\begin{array}{l}\text { location } \\
\text { sample } \\
\text { position }\end{array}$} & \multicolumn{6}{|c|}{ Svratka Unit } & \multicolumn{6}{|c|}{ Moldanubian Zone } & \multicolumn{4}{|c|}{ Kutná Hora Complex } \\
\hline & \multicolumn{2}{|c|}{ SV262 } & \multicolumn{2}{|c|}{ SV25 } & \multicolumn{2}{|c|}{$\operatorname{Cach} 2$} & \multicolumn{2}{|c|}{ КМ8А } & \multicolumn{2}{|c|}{ КМ8В } & \multicolumn{2}{|c|}{ КМ3М } & \multicolumn{2}{|c|}{ KMVSM2 } & \multicolumn{2}{|c|}{ KMVSM7 } \\
\hline & core & rim & core & rim & core & rim & core & rim & core & rim & core & rim & core & rim & core & rim \\
\hline \multicolumn{17}{|l|}{ (wt. \%) } \\
\hline $\mathrm{SiO}_{2}$ & 36.26 & 37.51 & 36.93 & 37.12 & 38.49 & 37.20 & 38.02 & 38.02 & 38.30 & 38.29 & 37.20 & 38.03 & 36.26 & 36.73 & 37.50 & 38.43 \\
\hline $\mathrm{TiO}_{2}$ & 0.19 & 0.05 & 0.11 & 0.10 & 0.25 & 0.22 & 0.09 & 0.18 & 0.58 & 0.48 & 0.28 & 0.31 & 0.70 & 0.00 & 0.35 & 0.18 \\
\hline $\mathrm{Cr}_{2} \mathrm{O}_{3}$ & 0.02 & 0.01 & 0.01 & 0.02 & 0.00 & 0.00 & 0.01 & 0.00 & 0.00 & 0.00 & 0.02 & 0.02 & 0.00 & 0.00 & 0.03 & 0.01 \\
\hline $\mathrm{Al}_{2} \mathrm{O}_{3}$ & 19.64 & 20.13 & 20.29 & 20.88 & 20.26 & 14.64 & 18.79 & 19.63 & 17.13 & 17.08 & 19.22 & 19.13 & 8.83 & 10.01 & 15.37 & 19.53 \\
\hline $\mathrm{FeO}$ & 22.43 & 27.32 & 22.34 & 30.96 & 12.06 & 17.67 & 16.96 & 15.22 & 9.02 & 9.14 & 9.13 & 10.94 & 21.15 & 16.40 & 18.12 & 13.80 \\
\hline $\mathrm{MnO}$ & 4.34 & 2.38 & 9.79 & 0.48 & 0.14 & 1.97 & 1.77 & 0.97 & 1.36 & 1.36 & 0.70 & 0.94 & 1.60 & 0.30 & 0.87 & 0.12 \\
\hline $\mathrm{MgO}$ & 0.11 & 0.37 & 0.42 & 1.47 & 0.08 & 0.15 & 0.22 & 0.36 & 0.34 & 0.39 & 0.03 & 0.05 & 0.04 & 0.05 & 0.25 & 0.60 \\
\hline $\mathrm{CaO}$ & 15.16 & 12.10 & 8.77 & 8.39 & 28.80 & 27.38 & 24.09 & 24.65 & 33.20 & 33.08 & 31.78 & 30.26 & 30.01 & 34.62 & 26.82 & 27.54 \\
\hline $\mathrm{Na}_{2} \mathrm{O}$ & 0.00 & 0.02 & 0.07 & 0.04 & 0.02 & 0.00 & 0.04 & 0.00 & 0.00 & 0.01 & 0.00 & 0.02 & 0.03 & 0.00 & 0.01 & 0.02 \\
\hline $\mathrm{K}_{2} \mathrm{O}$ & 0.00 & 0.00 & 0.00 & 0.00 & 0.06 & 0.00 & 0.13 & 0.30 & 0.12 & 0.01 & 1.12 & 0.93 & 0.00 & 0.50 & 0.00 & 0.00 \\
\hline Total & 98.14 & 99.88 & 98.74 & 99.46 & 100.15 & 99.24 & 100.13 & 99.34 & 100.06 & 99.84 & 99.47 & 100.64 & 98.62 & 98.61 & 99.32 & 100.22 \\
\hline \multicolumn{17}{|l|}{ (apfu)* } \\
\hline $\mathrm{Si}$ & 2.95 & 3.01 & 3.01 & 2.99 & 2.97 & 2.96 & 2.98 & 2.99 & 2.96 & 2.96 & 2.91 & 2.95 & 2.95 & 2.95 & 2.97 & 2.97 \\
\hline $\mathrm{Ti}$ & 0.01 & 0.00 & 0.01 & 0.01 & 0.01 & 0.01 & 0.01 & 0.01 & 0.03 & 0.03 & 0.02 & 0.02 & 0.04 & 0.00 & 0.02 & 0.01 \\
\hline $\mathrm{Cr}$ & 0.00 & 0.00 & 0.00 & 0.00 & 0.00 & 0.00 & 0.00 & 0.00 & 0.00 & 0.00 & 0.00 & 0.00 & 0.00 & 0.00 & 0.00 & 0.00 \\
\hline $\mathrm{Al}$ & 1.88 & 1.90 & 1.95 & 1.97 & 1.84 & 1.37 & 1.73 & 1.82 & 1.56 & 1.59 & 1.75 & 1.74 & 0.85 & 0.95 & 1.44 & 1.77 \\
\hline $\mathrm{Fe}^{2+}$ & 1.33 & 1.77 & 1.51 & 2.06 & 0.62 & 0.49 & 0.88 & 0.97 & 0.18 & 0.12 & 0.30 & 0.35 & 0.25 & 0.20 & 0.63 & 0.62 \\
\hline $\mathrm{Fe}^{3+}$ & 0.19 & 0.07 & 0.01 & 0.03 & 0.16 & 0.68 & 0.24 & 0.03 & 0.40 & 0.47 & 0.30 & 0.35 & 1.18 & 0.90 & 0.57 & 0.27 \\
\hline Mn & 0.30 & 0.16 & 0.68 & 0.03 & 0.01 & 0.13 & 0.12 & 0.07 & 0.09 & 0.04 & 0.05 & 0.06 & 0.11 & 0.02 & 0.06 & 0.01 \\
\hline $\mathrm{Mg}$ & 0.01 & 0.05 & 0.05 & 0.18 & 0.01 & 0.02 & 0.03 & 0.04 & 0.04 & 0.09 & 0.00 & 0.01 & 0.01 & 0.01 & 0.03 & 0.07 \\
\hline $\mathrm{Ca}$ & 0.00 & 1.04 & 0.77 & 0.72 & 2.38 & 2.33 & 2.02 & 2.08 & 2.75 & 2.74 & 2.66 & 2.51 & 2.61 & 2.98 & 2.28 & 2.28 \\
\hline $\mathrm{Na}$ & 1.32 & 0.00 & 0.01 & 0.01 & 0.00 & 0.00 & 0.01 & 0.00 & 0.00 & 0.00 & 0.00 & 0.00 & 0.01 & 0.00 & 0.00 & 0.00 \\
\hline $\mathrm{F}$ & 0.00 & 0.00 & 0.00 & 0.00 & 0.02 & 0.00 & 0.00 & 0.00 & 0.03 & 0.00 & 0.27 & 0.22 & 0.00 & 0.13 & 0.00 & 0.00 \\
\hline $\mathrm{X}_{\mathrm{Fe}}$ & 1.00 & 0.99 & 0.98 & 0.95 & 0.99 & 0.99 & 0.99 & 0.98 & 0.96 & 0.96 & 1.00 & 1.00 & 1.00 & 1.00 & 0.99 & 0.96 \\
\hline \multicolumn{17}{|l|}{$(\mathrm{mol} . \%)$} \\
\hline Alm & 37.70 & 57.87 & 49.33 & 68.79 & 14.48 & 13.87 & 26.80 & 21.27 & 2.24 & 3.67 & 8.86 & 12.08 & 7.15 & 0.00 & 15.48 & 16.84 \\
\hline Adr & 11.03 & 3.40 & 0.72 & 1.45 & 8.71 & 35.54 & 11.95 & 1.60 & 20.47 & 23.55 & 15.04 & 18.12 & 60.39 & 48.71 & 30.65 & 14.25 \\
\hline Grs & 39.31 & 31.74 & 25.26 & 22.70 & 76.17 & 45.37 & 56.37 & 73.28 & 72.91 & 68.30 & 74.37 & 67.43 & 28.55 & 50.33 & 50.65 & 66.15 \\
\hline Prp & 0.49 & 1.50 & 1.72 & 5.91 & 0.32 & 0.62 & 0.87 & 1.52 & 1.34 & 1.49 & 0.11 & 0.19 & 0.17 & 0.22 & 1.04 & 2.44 \\
\hline Sps & 11.41 & 5.46 & 22.95 & 1.08 & 0.33 & 4.60 & 3.98 & 2.33 & 3.03 & 2.99 & 1.56 & 2.11 & 3.75 & 0.74 & 2.10 & 0.29 \\
\hline
\end{tabular}

*recalculated on the basis of Rickwood (1968), $\mathrm{Fe}^{2+} / \mathrm{Fe}^{3+}$ was estimated according to Droop (1987)

Fig. 3 Photomicrographs of skarns showing crystallization relationships (a-d, f, g-BSE; e, h-plane polarized light). a - Svratka Unit, Svratouch: garnet-pyroxene skarn, garnet is surrounded by a decompression plagioclase-amphibole corona and encloses titanite; b - Svratka Unit, Kuklík: garnet-pyroxene skarn, disintegration of Jd-rich pyroxene to clinopyroxeneplagioclase symplectite; $\mathbf{c}$ - Svratka Unit, Věcov: garnet-pyroxene skarn, disintegration of titanite (Al-bearing) to titanite-plagioclase symplectite; d - Svratka Unit, Čachnov: garnet-pyroxene skarn, older garnet grains are overgrown by younger garnet rich in andradite component; $\mathbf{e}$ - Svratka Unit, Svratouch: grunerite-pyroxene skarn with garnet, garnets are surrounded by amphibole coronas, pyroxenes intergrown with grunerite; f-Moldanubian Zone, Budeč: garnet-pyroxene skarn, fractures in older Grs-rich garnets are filled by younger andradite; $\mathbf{g}$ - Kutná Hora Complex, Malešov: inhomogeneous grossular-andradite garnet with patchy zoning; h - Kutná Hora Complex, Malešov: garnet-pyroxene skarn with cleavage veinlets, filled with epidote and clinopyroxene. Abbreviations used: $\mathrm{Grt}=$ garnet, $\mathrm{Pl}=$ plagioclase, $\mathrm{Cpx}=$ clinopyroxene, $\mathrm{Hbl}=$ hornblende, $\mathrm{Ep}=$ epidote
(Fig. 3b) and disintegration of titanite (Al-bearing) to titanite-plagioclase symplectite, are frequent (Fig. 3c). Stage $\mathrm{M}_{3}$ produced the typical amphibole-plagioclase amphibolite-facies assemblage.

\subsection{Moldanubian Zone}

The following types of skarn were identified: garnetclinopyroxene skarn, garnetite, magnetite skarn with amphibole, and clinopyroxene skarn. The most rocks are massive, only clinopyroxene skarn is banded. The microscopic structure is nematoblastic and nematogranoblastic.

The major minerals, garnet and clinopyroxene, are accompanied by less abundant magnetite, quartz, pla- 


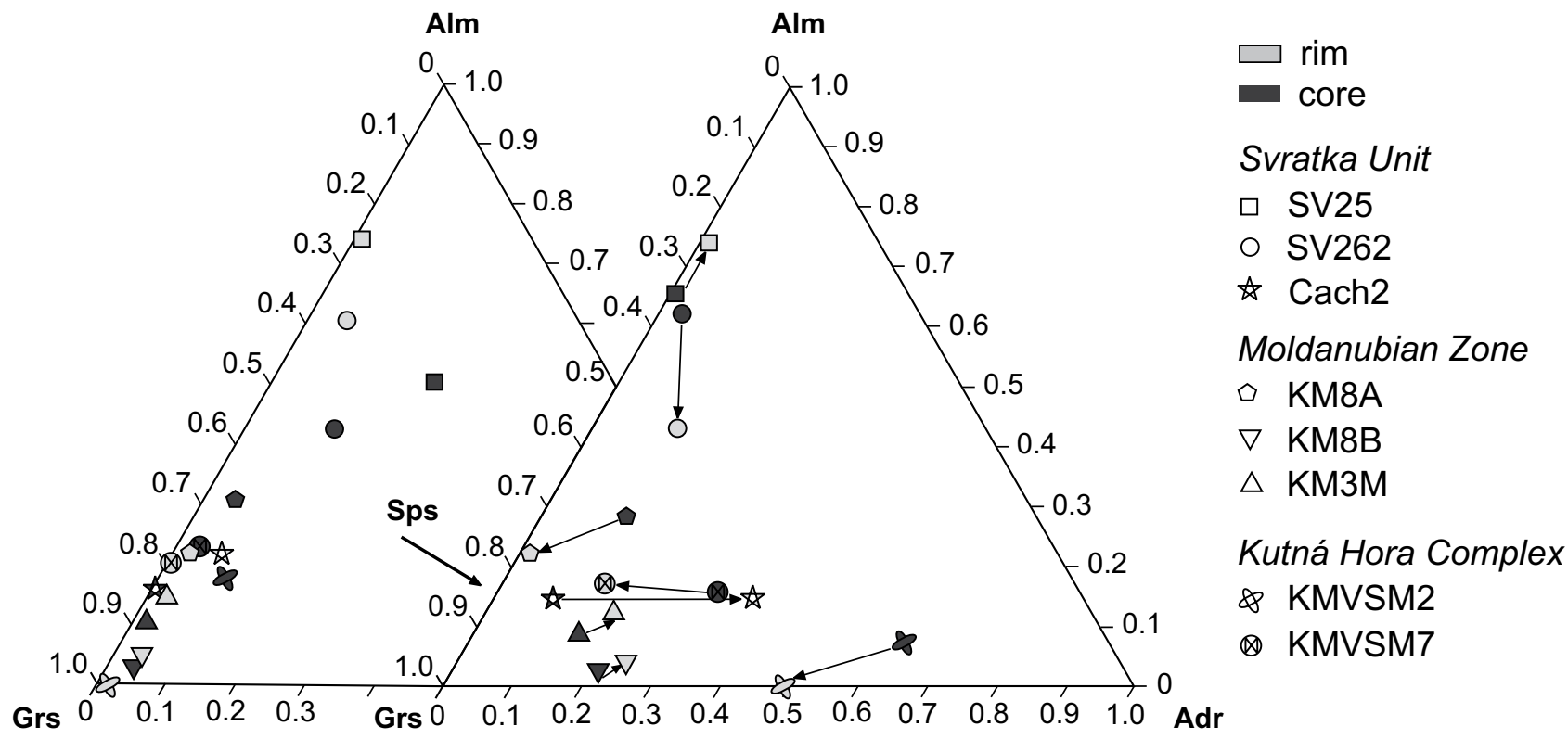

Fig. 4 Representative compositional trends for garnets from the Svratka Unit, the Moldanubian Zone and the Kutná Hora Complex. Arrows in the ternary diagrams indicate growth trends from the core to the rim.

Tab. 4 Representative chemical analyses of pyroxenes from the Svratka Unit, the Moldanubian Zone and the Kutná Hora Complex

\begin{tabular}{|c|c|c|c|c|c|c|c|c|c|c|c|c|c|}
\hline \multirow{3}{*}{$\begin{array}{l}\text { location } \\
\text { sample } \\
\text { position }\end{array}$} & \multicolumn{5}{|c|}{ Svratka Unit } & \multicolumn{3}{|c|}{ Moldanubian Zone } & \multicolumn{5}{|c|}{ Kutná Hora Complex } \\
\hline & \multirow{2}{*}{$\begin{array}{l}\text { SV262 } \\
\text { matrix }\end{array}$} & \multirow{2}{*}{$\begin{array}{c}\text { SV25 } \\
\text { matrix }\end{array}$} & \multirow{2}{*}{$\begin{array}{l}\text { KM7b } \\
\text { matrix }\end{array}$} & \multirow{2}{*}{$\begin{array}{c}\text { KMSB1 } \\
\text { inclusion }\end{array}$} & \multirow{2}{*}{$\begin{array}{l}\text { Cach2 } \\
\text { matrix }\end{array}$} & \multirow{2}{*}{$\begin{array}{l}\text { KM8A } \\
\text { matrix }\end{array}$} & \multirow{2}{*}{$\begin{array}{l}\text { KM8B } \\
\text { matrix }\end{array}$} & \multirow{2}{*}{$\begin{array}{l}\text { KM3A } \\
\text { matrix }\end{array}$} & \multirow{2}{*}{$\begin{array}{c}\text { KMVSM2 } \\
\text { matrix }\end{array}$} & \multicolumn{3}{|c|}{ KMVSM7 } & \multirow{2}{*}{$\begin{array}{l}\text { KMVSM4 } \\
\text { incl. in Grt }\end{array}$} \\
\hline & & & & & & & & & & core & rim & cleavage & \\
\hline \multicolumn{14}{|c|}{ (wt. \%) } \\
\hline $\mathrm{SiO}_{2}$ & 50.77 & 53.85 & 50.04 & 51.48 & 49.28 & 50.65 & 50.04 & 52.23 & 48.64 & 51.72 & 48.67 & 47.79 & 49.82 \\
\hline $\mathrm{TiO}_{2}$ & 0.00 & 0.11 & 0.02 & 0.11 & 0.06 & 0.11 & 0.12 & 0.08 & 0.04 & 0.00 & 0.09 & 0.14 & 0.03 \\
\hline $\mathrm{Al}_{2} \mathrm{O}_{3}$ & 0.38 & 6.63 & 0.87 & 4.88 & 1.15 & 0.84 & 2.64 & 1.25 & 0.69 & 0.67 & 2.08 & 2.19 & 0.47 \\
\hline $\mathrm{FeO}$ & 22.51 & 10.70 & 21.15 & 16.04 & 20.03 & 18.75 & 12.99 & 12.31 & 25.02 & 13.37 & 20.03 & 21.76 & 22.62 \\
\hline $\mathrm{MnO}$ & 0.20 & 0.03 & 0.27 & 0.13 & 1.01 & 0.32 & 0.51 & 1.15 & 1.12 & 0.22 & 0.31 & 0.36 & 0.82 \\
\hline $\mathrm{MgO}$ & 5.43 & 7.92 & 4.48 & 5.70 & 5.06 & 6.68 & 9.32 & 10.59 & 1.98 & 9.23 & 5.07 & 3.83 & 3.70 \\
\hline $\mathrm{CaO}$ & 20.53 & 17.06 & 21.55 & 16.99 & 21.84 & 23.62 & 24.16 & 23.00 & 22.57 & 23.55 & 22.75 & 22.27 & 22.42 \\
\hline $\mathrm{Na}_{2} \mathrm{O}$ & 0.68 & 4.48 & 0.88 & 4.00 & 0.64 & 0.09 & 0.20 & 0.22 & 0.34 & 0.49 & 0.59 & 0.61 & 0.33 \\
\hline $\mathrm{K}_{2}{ }_{2}^{2} \mathrm{O}$ & 0.00 & 0.00 & 0.00 & 0.00 & 0.10 & 0.00 & 0.01 & 0.01 & 0.00 & 0.00 & 0.00 & 0.00 & 0.00 \\
\hline Total & 100.50 & 100.78 & 99.26 & 99.33 & 99.16 & 101.05 & 99.99 & 100.83 & 100.40 & 99.25 & 99.59 & 98.95 & 100.21 \\
\hline \multicolumn{14}{|l|}{ (apfu)* } \\
\hline $\mathrm{Si}$ & 2.00 & 1.97 & 1.99 & 1.96 & 1.96 & 1.97 & 1.91 & 1.97 & 1.96 & 1.99 & 1.92 & 1.92 & 1.99 \\
\hline $\mathrm{Ti}$ & 0.00 & 0.00 & 0.00 & 0.00 & 0.00 & 0.00 & 0.00 & 0.00 & 0.00 & 0.00 & 0.00 & 0.00 & 0.00 \\
\hline $\mathrm{Al}$ & 0.02 & 0.28 & 0.04 & 0.21 & 0.18 & 0.04 & 0.09 & 0.05 & 0.03 & 0.03 & 0.10 & 0.10 & 0.01 \\
\hline $\mathrm{Fe}^{2+}$ & 0.70 & 0.23 & 0.66 & 0.35 & 0.59 & 0.58 & 0.42 & 0.38 & 0.78 & 0.41 & 0.56 & 0.63 & 0.73 \\
\hline $\mathrm{Fe}^{3+}$ & 0.04 & 0.09 & 0.04 & 0.16 & 0.08 & 0.03 & 0.00 & 0.01 & 0.07 & 0.03 & 0.10 & 0.10 & 0.02 \\
\hline $\mathrm{Mn}$ & 0.01 & 0.00 & 0.01 & 0.00 & 0.03 & 0.01 & 0.02 & 0.04 & 0.04 & 0.01 & 0.01 & 0.01 & 0.03 \\
\hline $\mathrm{Mg}$ & 0.32 & 0.43 & 0.27 & 0.32 & 0.30 & 0.39 & 0.53 & 0.60 & 0.12 & 0.53 & 0.30 & 0.23 & 0.22 \\
\hline $\mathrm{Ca}$ & 0.87 & 0.67 & 0.92 & 0.69 & 0.93 & 0.98 & 0.99 & 0.93 & 0.98 & 0.97 & 0.96 & 0.96 & 0.96 \\
\hline $\mathrm{Na}$ & 0.05 & 0.32 & 0.07 & 0.30 & 0.05 & 0.01 & 0.02 & 0.02 & 0.03 & 0.04 & 0.05 & 0.05 & 0.03 \\
\hline $\mathrm{K}$ & 0.00 & 0.00 & 0.00 & 0.00 & 0.01 & 0.00 & 0.00 & 0.00 & 0.00 & 0.00 & 0.00 & 0.00 & 0.00 \\
\hline \multicolumn{14}{|l|}{$(\mathrm{mol} \%)$} \\
\hline Wo & 44.81 & 47.00 & 48.42 & 45.00 & 48.19 & 49.41 & 50.68 & 47.68 & 49.35 & 49.81 & 49.81 & 49.64 & 48.88 \\
\hline En & 16.49 & 30.00 & 14.01 & 21.00 & 15.55 & 19.45 & 27.20 & 30.53 & 6.01 & 27.15 & 15.43 & 11.87 & 11.21 \\
\hline Fs & 38.70 & 23.00 & 37.57 & 34.00 & 36.27 & 31.14 & 22.12 & 21.80 & 44.64 & 23.03 & 34.76 & 38.49 & 39.91 \\
\hline WEF & 94.81 & 68.00 & 93.14 & 70.00 & 94.98 & 99.34 & 98.50 & 98.38 & 97.31 & 96.35 & 95.35 & 95.10 & 97.43 \\
\hline $\mathrm{Jd}$ & 1.56 & 24.00 & 3.03 & 16.00 & 0.75 & 0.06 & 1.50 & 1.88 & 0.00 & 1.29 & 0.76 & 0.81 & 0.85 \\
\hline Aeg & 3.64 & 9.00 & 3.79 & 14.00 & 4.26 & 0.60 & 0.00 & 0.44 & 2.69 & 2.37 & 3.89 & 4.08 & 1.72 \\
\hline
\end{tabular}

incl.=inclusion

*recalculated on the basis of Cawthorn and Collerson (1974), $\mathrm{Fe}^{2+} / \mathrm{Fe}^{3+}$ was estimated by charge balance proposed by the IMA guidelines 


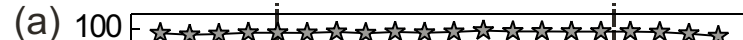

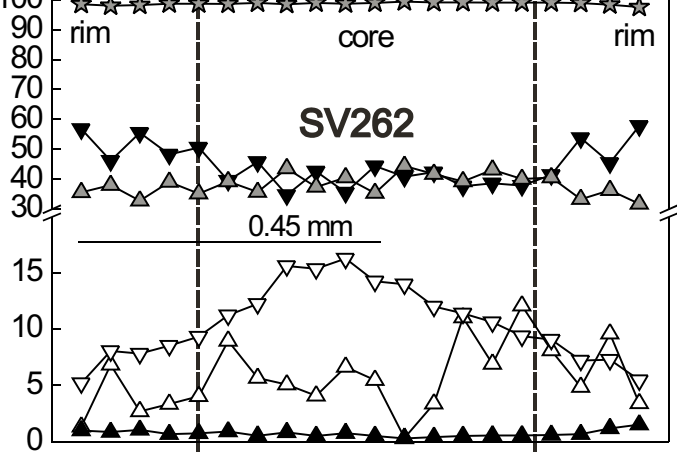

(c)

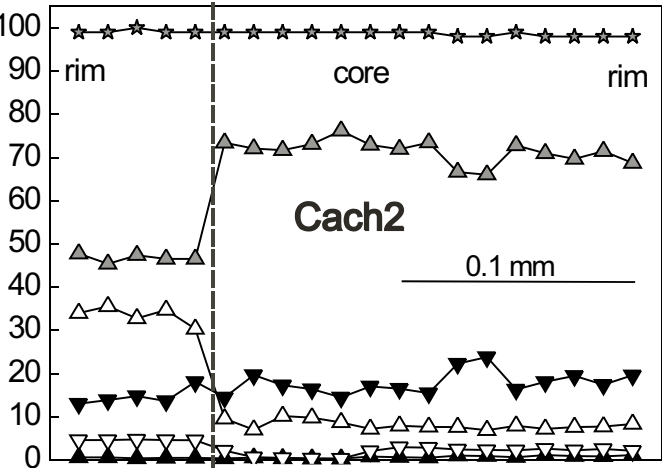

(e)

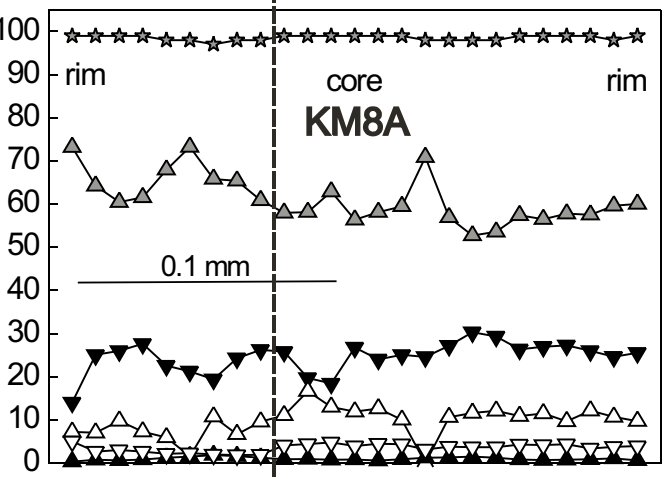

(g)

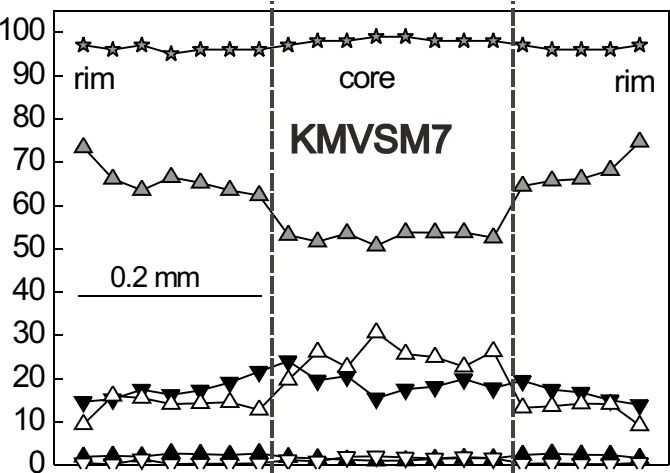

(b)



(d)

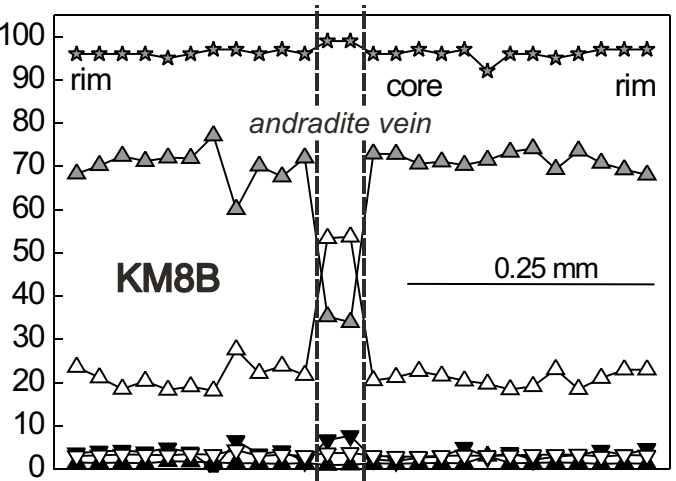

(f)

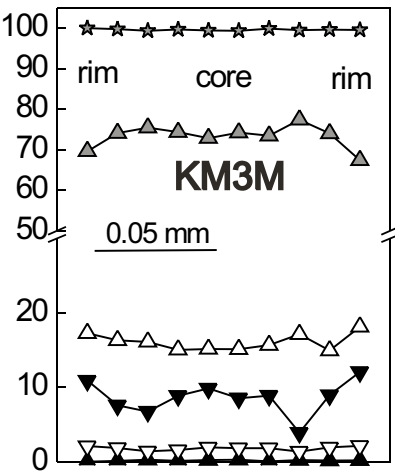

(h)





Fig. 5 Representative garnet profiles (mol \%): a-c - Svratka Unit, Svratouch, Čachnov; d-f -Moldanubian Zone, Budeč, Vepřová; g-h - Kutná Hora Complex, Malešov. 


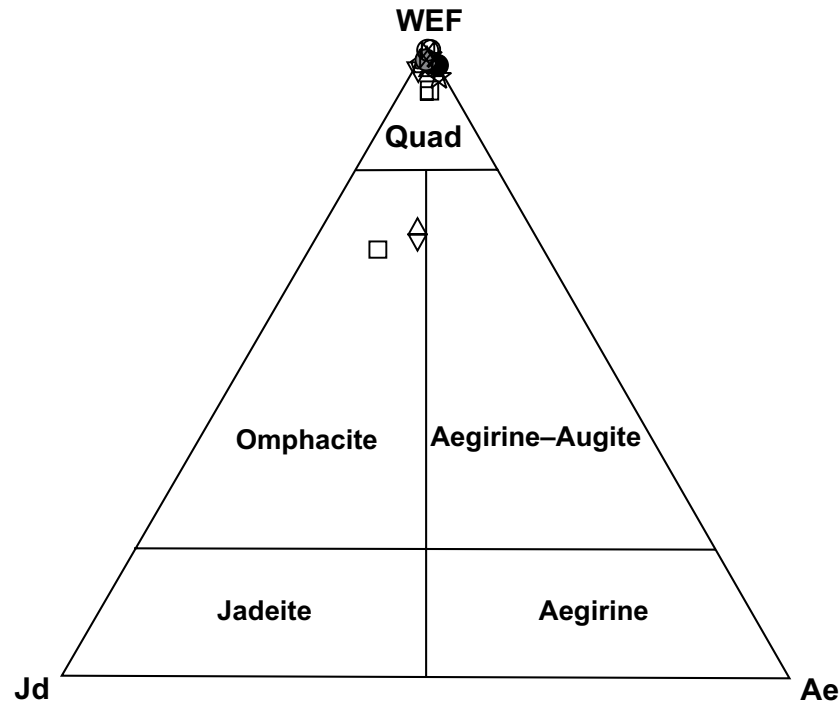

Svratka Unit
$\square \quad$ SV25
$\circ \quad$ SV262
$\square \quad \mathrm{KM} 7$
$\forall \quad \mathrm{KMSB} 21$
$\star$ Cach2

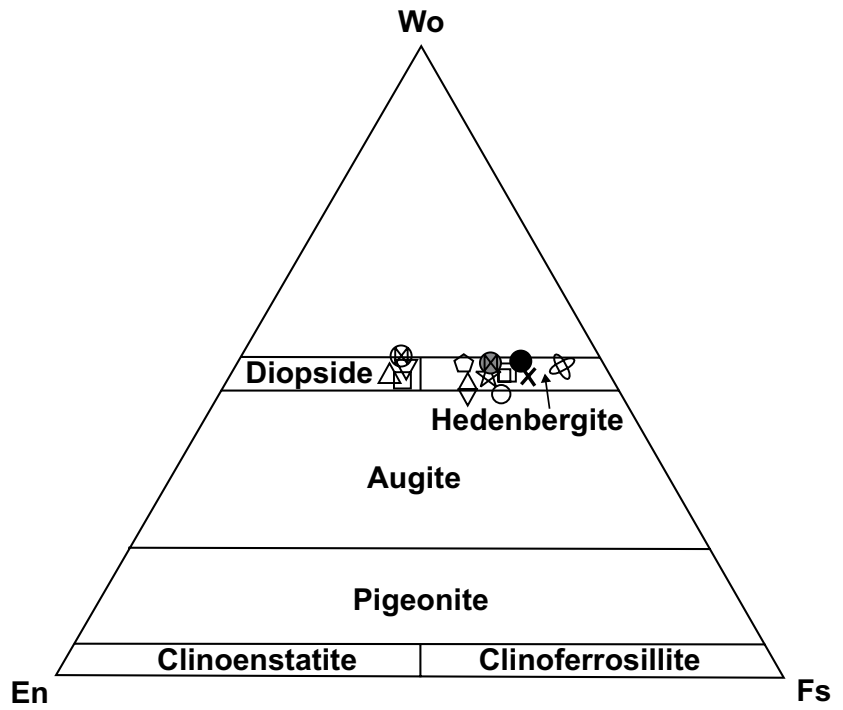

Kutná Hora Complex

$\otimes$ KMVSM2

$\otimes$ KMVSM7 core

¿ KMVSM7 rim

- KMVSM7 cleavage $x$ KMVSM4

Fig. 6 Representative chemical compositions of pyroxenes from the Svratka Unit, the Moldanubian Zone and the Kutná Hora Complex in the classification diagram of Cawthorn and Collerson (1974).

Tab. 5 Representative chemical analyses of amphiboles from the Svratka Unit, the Moldanubian Zone and the Kutná Hora Complex

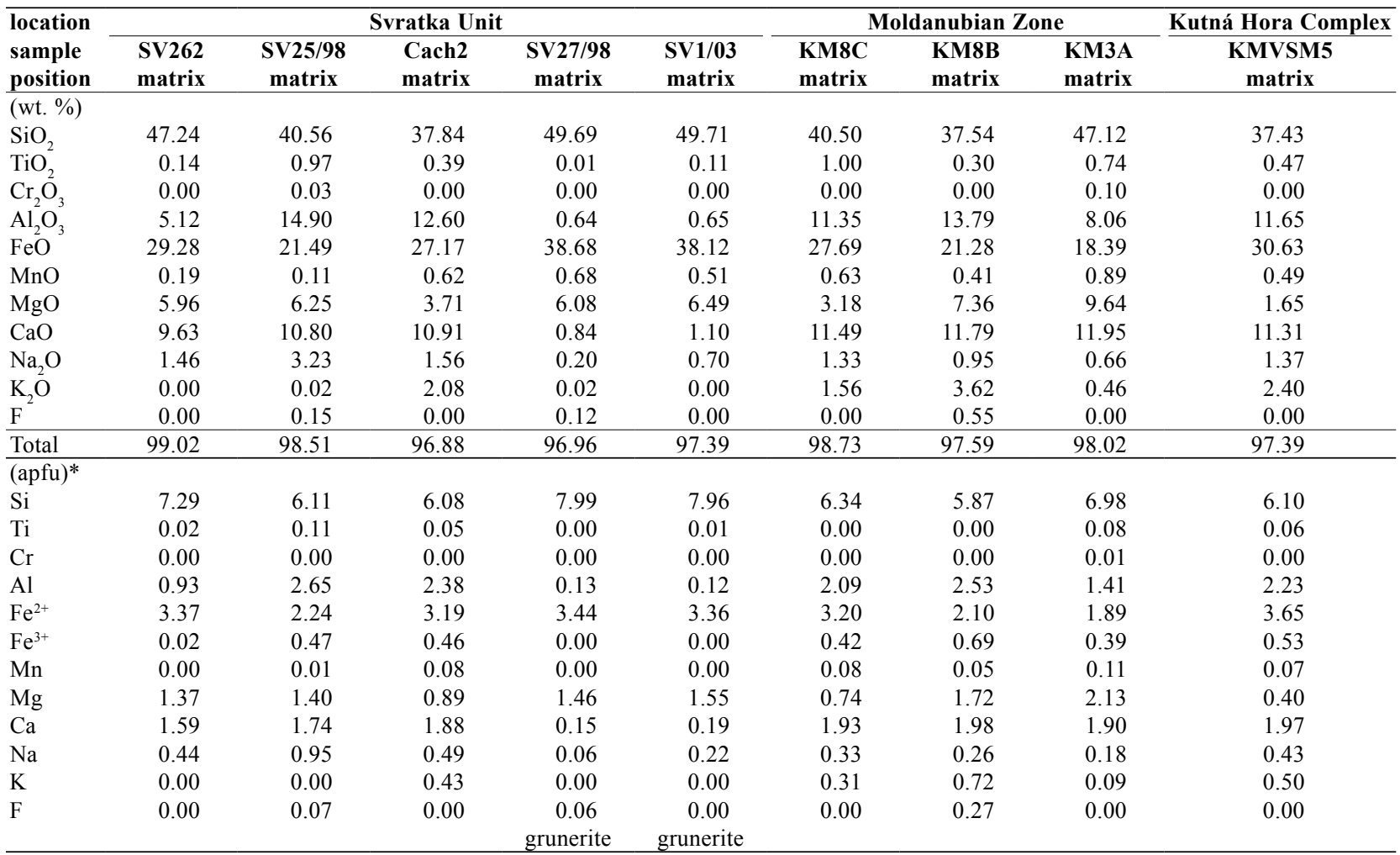

*recalculated on the basis of Richard and Clarke (1990), $\mathrm{Fe}^{2+} / \mathrm{Fe}^{3+}$ was estimated according to Robinson et al. (1981) 
gioclase (bytownite to anorthite), epidote, clinozoisite and amphibole (hastingsite, grunerite). Titanite and apatite are the common accessories. Opaque minerals include a number of mineral species. The most common mineral is magnetite, corresponding to 20 vol. \% in some samples. Pyrrhotite, pyrite and arsenopyrite are minor components. Rare löllingite and native bismuth are identified as inclusions in arsenopyrite.

The results of petrological study of skarns in the Moldanubian Zone (Holšice, Zliv, Vápenka, and Vlastějovice localities) were reported by Potužák (1996) and Drahota et al. (2005), data from MZ in south-western Moravia (Županovice) by Pertoldová (1986), and from the Gföhl Unit in MZ (Slatina and Rešice) by Pertold et al. (1997).

\subsubsection{Garnet}

Garnet forms subhedral grains, which exhibit irregular compositional zoning. The compositional inhomogeneity is depicted in Figs 4, $5 \mathrm{~d}-\mathrm{f}$. It includes variations in grossular $(\sim 50$ to $80 \mathrm{~mol} . \%)$, almandine ( 1 to $30 \mathrm{~mol} . \%)$ and andradite $(\sim 1$ to $30 \mathrm{~mol} . \%)$ components. In some instances, there is an outer rim of nearly pure grossular mantling the garnet grains. Some garnets contain fluorine in significant amounts (0.35-1.12 wt. \%); the highest contents were recorded in grossularrich outer rims. Garnet-pyroxene skarn with magnetite, sample KM8B03, contains two garnet generations with contrasting optical and compositional characteristics. The older garnet grains with predominating grossular component $\left(\mathrm{Grs}_{77-60} \mathrm{Alm}_{1-5} \mathrm{Sps}_{3} \mathrm{Adr}_{8-24} \operatorname{Prp}_{1-2}, \mathrm{X}_{\mathrm{Fe}}=0.95-0.97\right.$ mol. \%) are corroded and fractured. The fractures are filled by a younger garnet with increased andradite component $\left(\mathrm{Grs}_{34-35} \mathrm{Alm}_{6-8} \mathrm{Sps}_{4} \mathrm{Adr}_{53-54} \operatorname{Prp}_{1}, \mathrm{X}_{\mathrm{Fe}}=0.99\right.$ mol. \%; Figs 3f, 5d, Tab. 3). Garnet in sample KM3M is compositionally rather inhomogeneous and exhibits slight retrograde zoning, especially in the contents of grossular, almandine and andradite (from core to rim $\mathrm{Grs}_{74-67} \mathrm{Alm}_{9-12}$ $\operatorname{Adr}_{15-18}$, Fig. 5f, Tab. 6).

\subsubsection{Clinopyroxene and amphibole}

Clinopyroxenes belong to the hedenbergite-diopside group (Fig. 6). Replacement of clinopyroxene by secondary amphibole with hastingsite composition is common (Tab. 5).

\subsubsection{Plagioclase and titanite}

Compositionally homogeneous calcic plagioclase occurs in minor amounts interstitially among garnet and clinopyroxene grains. It contains over $90 \mathrm{~mol} \%$ of anorthite component (Tab. 5). Titanite is compositionally heterogeneous. The malayaite component is present in some samples, as indicated by increased tin (up to 1.23 wt. \% SnO), and fluorine contents (up to 0.52 wt. \%) (Tab. 7).

\subsubsection{Metamorphic development}

The chemical composition of garnet in skarns indicates weak retrograde zoning. The conditions of amphibolitefacies metamorphism presumably varied during the crystallization of skarn minerals, as probably did the oxygen and fluorine fugacity. The oxygen fugacity was probably increased only locally. 
Tab. 7 Representative chemical analyses of titanites from the Svratka Unit, the Moldanubian Zone and the Kutná Hora Complex

\begin{tabular}{|c|c|c|c|c|c|c|c|c|}
\hline \multirow{2}{*}{$\begin{array}{l}\text { location } \\
\text { sample } \\
\text { position }\end{array}$} & \multicolumn{5}{|c|}{ Svratka Unit } & \multicolumn{2}{|c|}{ Moldanubian Zone } & \multirow{2}{*}{$\begin{array}{c}\text { Kutná Hora Complex } \\
\text { KMV20A } \\
\text { matrix }\end{array}$} \\
\hline & $\begin{array}{c}\text { SV25/98 } \\
\text { matrix }\end{array}$ & $\begin{array}{c}\text { KMSB21c } \\
\text { pure titanite }\end{array}$ & $\begin{array}{c}\text { KMSB21c } \\
\text { pure titanite }\end{array}$ & $\begin{array}{c}\text { KMSB21c } \\
\text { symplectite }\end{array}$ & $\begin{array}{c}\text { KMSB21c } \\
\text { symplectite }\end{array}$ & $\begin{array}{l}\text { KM3A } \\
\text { matrix }\end{array}$ & $\begin{array}{l}\text { KM3C } \\
\text { matrix }\end{array}$ & \\
\hline \multicolumn{9}{|l|}{ (wt. \%) } \\
\hline $\mathrm{SiO}_{2}$ & 31.07 & 31.37 & 30.69 & 30.05 & 30.20 & 30.26 & 30.40 & 29.91 \\
\hline $\mathrm{TiO}_{2}$ & 37.30 & 31.24 & 31.51 & 34.92 & 34.71 & 36.70 & 29.02 & 36.19 \\
\hline $\mathrm{Cr}_{2} \mathrm{O}_{3}$ & 0.00 & 0.00 & 0.01 & 0.00 & 0.00 & 0.00 & 0.00 & 0.03 \\
\hline $\mathrm{Al}_{2} \mathrm{O}_{3}$ & 3.01 & 5.47 & 4.72 & 1.76 & 1.91 & 3.28 & 7.38 & 2.67 \\
\hline $\mathrm{FeO}$ & 1.26 & 1.06 & 0.98 & 0.76 & 0.75 & 0.79 & 0.69 & 1.19 \\
\hline $\mathrm{MnO}$ & 0.15 & 0.04 & 0.01 & 0.00 & 0.00 & 0.19 & 0.00 & 0.02 \\
\hline $\mathrm{MgO}$ & 0.00 & 0.01 & 0.01 & 0.00 & 0.00 & 0.00 & 0.00 & 0.00 \\
\hline $\mathrm{CaO}$ & 28.17 & 29.24 & 28.84 & 28.20 & 28.29 & 28.94 & 30.21 & 29.02 \\
\hline $\mathrm{Na}_{2} \mathrm{O}$ & 0.02 & 0.00 & 0.04 & 0.04 & 0.01 & 0.01 & 0.00 & 0.00 \\
\hline $\mathrm{ZrO}_{2}$ & 0.00 & 0.00 & 0.00 & 0.01 & 0.02 & 0.00 & 0.00 & 0.00 \\
\hline $\mathrm{Nb}_{2} \mathrm{O}_{5}$ & 0.00 & 0.07 & 0.12 & 0.12 & 0.10 & 0.11 & 0.09 & 0.00 \\
\hline $\mathrm{Ta}_{2} \mathrm{O}_{5}$ & 0.00 & 0.00 & 0.00 & 0.07 & 0.04 & 0.20 & 0.00 & 0.00 \\
\hline $\mathrm{F}$ & 0.00 & 1.51 & 1.31 & 0.47 & 0.52 & 0.00 & 0.52 & 0.00 \\
\hline $\mathrm{V}_{2} \mathrm{O}_{5}$ & 0.00 & 0.05 & 0.00 & 0.00 & 0.00 & 0.20 & 0.18 & 0.00 \\
\hline $\mathrm{SnO}$ & 0.00 & 0.11 & 1.51 & 2.67 & 2.57 & 0.00 & 1.23 & 0.00 \\
\hline Total & 100.98 & 100.17 & 99.75 & 99.07 & 99.12 & 100.68 & 99.72 & 99.03 \\
\hline \multicolumn{9}{|l|}{ (apfu)* } \\
\hline $\mathrm{Si}$ & 4.01 & 3.82 & 3.81 & 3.92 & 3.92 & 3.87 & 3.79 & 3.96 \\
\hline $\mathrm{Ti}$ & 3.62 & 2.86 & 2.94 & 3.42 & 3.39 & 3.53 & 2.72 & 3.60 \\
\hline $\mathrm{Cr}$ & 0.00 & 0.00 & 0.00 & 0.00 & 0.00 & 0.00 & 0.00 & 0.00 \\
\hline $\mathrm{Al}$ & 0.46 & 0.79 & 0.69 & 0.27 & 0.29 & 0.49 & 1.08 & 0.42 \\
\hline $\mathrm{Fe}^{2+}$ & 0.14 & 0.11 & 0.10 & 0.08 & 0.08 & 0.08 & 0.07 & 0.13 \\
\hline $\mathrm{Fe}^{3+}$ & 0.00 & 0.00 & 0.00 & 0.00 & 0.00 & 0.00 & 0.00 & 0.00 \\
\hline $\mathrm{Mn}$ & 0.02 & 0.00 & 0.00 & 0.00 & 0.00 & 0.02 & 0.00 & 0.00 \\
\hline $\mathrm{Mg}$ & 0.00 & 0.00 & 0.00 & 0.00 & 0.00 & 0.00 & 0.00 & 0.00 \\
\hline $\mathrm{Ca}$ & 3.90 & 3.82 & 3.84 & 3.94 & 3.94 & 3.96 & 4.04 & 4.12 \\
\hline $\mathrm{Na}$ & 0.01 & 0.00 & 0.01 & 0.01 & 0.00 & 0.00 & 0.00 & 0.00 \\
\hline $\mathrm{Zr}$ & 0.00 & 0.00 & 0.00 & 0.00 & 0.00 & 0.00 & 0.00 & 0.00 \\
\hline $\mathrm{Nb}$ & 0.00 & 0.00 & 0.01 & 0.01 & 0.01 & 0.01 & 0.01 & 0.00 \\
\hline $\mathrm{Ta}$ & 0.00 & 0.00 & 0.00 & 0.00 & 0.00 & 0.01 & 0.00 & 0.00 \\
\hline $\mathrm{F}$ & 0.00 & 0.04 & 0.03 & 0.01 & 0.01 & 0.00 & 0.01 & 0.00 \\
\hline $\mathrm{V}$ & 0.00 & 0.00 & 0.00 & 0.00 & 0.00 & 0.02 & 0.01 & 0.00 \\
\hline$\underline{\mathrm{Sn}}$ & 0.00 & 0.01 & 0.08 & 0.16 & 0.15 & 0.00 & 0.07 & 0.00 \\
\hline
\end{tabular}

*recalculated on the basis of Deer et al. $(1966,1972)$

\subsection{Kutná Hora Complex}

Inhomogeneous lenses and bodies of skarn in the Kutná Hora Complex are located in variously migmatized two-mica gneisses, some garnet bearing. These skarns are massive, fine-grained rocks with compact, banded or schlieren-like structure. The texture is granoblastic or nemato-granoblastic. The following skarn types were identified: garnet-pyroxene skarns, pyroxene skarns, pyroxene skarns with garnet, pyroxene-garnet skarns grading to garnetites. Garnet-pyroxene skarn is the most abundant type. Clinopyroxenes in these rocks are partly or strongly replaced by amphiboles. The typical mineral assemblage is hedenbergite, grossular-andradite garnet, epidote and magnetite. Accessory minerals are apatite, titanite, allanite and cerite. Secondary minerals are represented by amphiboles and four generations of epidote.

\subsubsection{Garnet}

Garnet forms clusters or irregular layers. Based on composition, two groups of garnets are distinguished (Figs 4, 5g-h). The first group includes three-component andradite-grossular-almandine garnets (Fig. $5 \mathrm{~g}$ ) formed by blastic growth in epidosite layers; the second group contains grossular-andradite garnets (Fig. 3g, 5h). Prograde zoning in three-component garnets is expressed by variation in the abundances of all three components (from core to rim $\mathrm{Grs}_{50-75} \mathrm{Alm}_{24-15} \mathrm{Sps}_{2-0} \mathrm{Adr}_{31-9} \operatorname{Prp}_{1-3}, \mathrm{X}_{\mathrm{Fe}}=$ 0.99-0.96 mol. \%, Fig. 5g, Tab. 3). The three-component 
garnets enclose epidote and clinopyroxene inclusions. In some cases, garnets are affected by secondary replacement by younger epidote. Garnets of the second group also exhibit compositional zoning, even though the individual grains are often compositionally inhomogeneous. There is a general tendency to andradite enrichment in the crystal cores and to a grossular increase toward rims (from core to rim $\mathrm{Grs}_{24-65} \mathrm{Alm}_{9-0} \mathrm{Sps}_{4-0} \mathrm{Adr}_{65-33}$, Fig. 5h, Tab. 3). Younger grossular-rich garnet follows interfaces among grains. Hydrogrossular containing up to $0.46 \mathrm{wt}$. $\%$ fluorine is also a two-component garnet.

\subsubsection{Clinopyroxene}

Clinopyroxene is the most abundant mineral. Three generations of clinopyroxene are distinguished. Hedenbergite, as the oldest pyroxene phase, is enclosed in garnet and contains $c .1 \mathrm{~mol} . \%$ of the jadeite component. The second generation includes matrix clinopyroxenes, which are unzoned, or rarely show a weak compositional zoning. The crystal cores have diopside composition, while the rims are enriched in $\mathrm{FeO}(20-22 \%)$ and can be classified as hedenbergite (Fig. 6, Tab. 4). The youngest generation of clinopyroxene fills cleavage in the form of veinlets (Fig. 3h). It has hedenbergite composition (Fig. 6, Tab. 4).

\subsubsection{Plagioclase and amphibole}

Both plagioclase and amphibole are rare and only secondary. Plagioclase is oligoclase $\left(\sim \mathrm{An}_{27} ; \mathrm{Tab} .6\right)$; amphibole corresponds to hastingsite (Tab. 5).

\subsubsection{Epidote}

Microscopic study resulted in identification of five generations of epidote-clinozoisite series minerals. Probably the oldest is epidote I in epidosite bands in sample KMVSM7, which shows replacement of epidote by garnet. This newly formed garnet contains inclusions of epidote I. Epidote of the second generation occurs as euhedral crystals enclosing, in their cores, allanite (cerite?). This may represent a phase enriched in REE, released during replacement of epidote I by garnet in epidosite layers. Epidote III was produced by replacement of certain compositional zones in garnet, leaving behind atoll-like (hollow) garnet crystals. The youngest epidote IV occurs together with clinopyroxene, filling the cleavage in the form of veinlets (Fig. 3h). The table of representative epidote analyses (Tab. 8) contains data for all four epidote generations.

Epidotes are typically free of compositional zoning. The chemical composition of epidote enclosed in garnet, epidote formed via replacement of garnet, and epidote in late cleavage veinlets (I, II, IV) shows very limited variation $(\mathrm{Si}=2.96-2.99, \mathrm{Al} \sim 2.25 \mathrm{apfu}$; Tab. 8). Epidotes enriched in REE contain up to 5 wt. $\% \mathrm{Ce}_{2} \mathrm{O}_{3}, 3$ wt. \% $\mathrm{La}_{2} \mathrm{O}_{3}$, and 2 wt. $\% \mathrm{Nd}_{2} \mathrm{O}_{3}$. The contents of $\mathrm{ThO}_{2}, \mathrm{UO}_{2}$, $\mathrm{Sm}_{2} \mathrm{O}_{3}$ and $\mathrm{F}$ are lower than 1 wt. \%.

\subsubsection{Metamorphic development}

Garnets in skarns in the Kutná Hora Complex are rather compositionally heterogeneous, including variation in the grossular and andradite components (Fig. $5 \mathrm{~g}-\mathrm{h}$ ). The relative abundance of mineral phases rich in REE (mainly metamict allanite) is probably related to enrichment in REE in the precursor epidosite bands. These layers were probably derived by hydrothermal alteration of $\mathrm{Ca}$ - and Fe-enriched layers. Due to the extensive stability field of epidote in amphibolite facies, epidosites persisted up to a stage of epidote replacement by garnet. The prograde compositional zoning was observed in some garnets (Fig. $5 \mathrm{~g}$ ). The andradite component in the garnet indicates fluctuations in oxygen fugacity (Fig. 5h). The rather constant composition of the individual generations of pyroxene and epidote, belonging to the successive stages of metamorphism, suggests that variation in the P-T conditions was small.

\section{Whole-rock geochemistry of the skarns}

Altogether 59 whole-rock samples were used for chemical characterization of skarns in the studied units. The samples are listed in Tab. 1; major- and trace-element analyses are presented in Tabs 9-11.

\subsection{Svratka Unit}

Skarns from this unit are represented by samples from 13 localities. The mineral assemblages are dominated by Grt-Cpx, Cpx and Grt-Amph. The analyses of major and trace elements are presented in Tab. 9. The abundances of the major elements are highly variable: $35-55$ wt. \% $\mathrm{SiO}_{2}, 1-18$ wt. $\% \mathrm{Al}_{2} \mathrm{O}_{3}, 1-23$ wt. \% $\mathrm{CaO}, 2-47$ wt. \% $\mathrm{Fe}_{2} \mathrm{O}_{3}$ and 2-8 wt. \% $\mathrm{MgO}$ (Fig. 10).

The chondrite-normalized REE patterns for most of the samples resemble those for post-Archaean sediments (Taylor and McLennan 1985; Condie 1993). Overall, the abundances of the trace elements, including REE, are highly variable. Skarns from the Svratka Unit match the skarns from the Moldanubian Zone in their geochemical characteristics. A positive Eu anomaly is present in samples 35 and 36 from Teplá $\left(\mathrm{Eu} / \mathrm{Eu}^{*}=1.94\right.$ and 1.39), 49 from Čachnov $\left(\mathrm{Eu} / \mathrm{Eu}^{*}=1.99\right)$ and 52 from Ruda $\left(\mathrm{Eu} / \mathrm{Eu}^{*}=1.19\right)$. Samples 57 (Pernštejn) and 42 and 
Tab. 8 Representative chemical analyses of epidotes from the Svratka Unit, the Moldanubian Zone and the Kutná Hora Complex

\begin{tabular}{|c|c|c|c|c|c|c|c|c|}
\hline \multirow{3}{*}{$\begin{array}{l}\text { location } \\
\text { sample } \\
\text { position }\end{array}$} & \multirow{3}{*}{$\begin{array}{c}\text { Svratka Unit } \\
\text { Cach2 } \\
\text { matrix }\end{array}$} & \multirow{3}{*}{$\begin{array}{c}\text { Moldanubian Zone } \\
\text { KM3E } \\
\text { matrix }\end{array}$} & \multicolumn{4}{|c|}{ Kutná Hora Complex } & \multicolumn{2}{|c|}{ Kutná Hora Complex } \\
\hline & & & \multirow{2}{*}{$\begin{array}{c}\text { KMVSM7 } \\
\text { phase I. }\end{array}$} & \multirow{2}{*}{$\begin{array}{c}\text { KMVSM1 } \\
\text { after Grt con. }\end{array}$} & \multicolumn{2}{|c|}{ KMVSM7 } & & \multirow{2}{*}{$\begin{array}{l}\text { KMVSM1 } \\
\text { with REE }\end{array}$} \\
\hline & & & & & incl.in Grt & cleavage & & \\
\hline (wt. \%) & & & & & & & (wt. \%) & \\
\hline $\mathrm{SiO}_{2}$ & 37.80 & 39.22 & 37.61 & 37.81 & 37.53 & 37.49 & $\mathrm{SiO}_{2}$ & 39.62 \\
\hline $\mathrm{TiO}_{2}$ & 0.00 & 0.06 & 0.00 & 0.00 & 0.00 & 0.00 & $\mathrm{TiO}_{2}$ & 0.25 \\
\hline $\mathrm{Cr}_{2} \mathrm{O}_{3}$ & 0.00 & 0.04 & 0.00 & 0.00 & 0.00 & 0.00 & $\mathrm{Al}_{2} \mathrm{O}_{3}$ & 12.93 \\
\hline $\mathrm{FeO}$ & 10.58 & 10.20 & 11.47 & 11.65 & 11.16 & 11.53 & $\mathrm{MnO}$ & 0.66 \\
\hline $\mathrm{MnO}$ & 0.00 & 0.08 & 0.00 & 0.00 & 0.00 & 0.00 & $\mathrm{MgO}$ & 0.13 \\
\hline $\mathrm{MgO}$ & 0.00 & 0.00 & 0.03 & 0.00 & 0.02 & 0.01 & $\mathrm{La}_{2} \mathrm{O}_{3}$ & 3.04 \\
\hline $\mathrm{CaO}$ & 22.55 & 23.32 & 24.24 & 23.70 & 24.50 & 23.86 & $\mathrm{Ce}_{2} \mathrm{O}_{3}$ & 5.28 \\
\hline $\mathrm{Na}_{2} \mathrm{O}$ & 0.00 & 0.01 & 0.00 & 0.00 & 0.00 & 0.00 & $\mathrm{Pr}_{2} \mathrm{O}_{3}$ & 0.58 \\
\hline $\mathrm{K}_{2} \mathrm{O}$ & 0.00 & 0.00 & 0.00 & 0.00 & 0.00 & 0.00 & $\mathrm{Nd}_{2} \mathrm{O}_{3}$ & 2.03 \\
\hline $\mathrm{Cl}$ & 0.00 & 0.00 & 0.00 & 0.00 & 0.00 & 0.00 & $\mathrm{ThO}_{2}$ & 0.55 \\
\hline Total & 95.28 & 98.85 & 97.66 & 97.17 & 97.43 & 96.90 & $\mathrm{UO}_{2}$ & 0.22 \\
\hline (apfu) & & & & & & & $\mathrm{CaO}$ & 12.58 \\
\hline $\mathrm{Si}$ & 3.03 & 3.02 & 2.96 & 2.99 & 2.97 & 2.98 & $\mathrm{P}_{2} \mathrm{O}_{5}$ & 0.05 \\
\hline $\mathrm{Ti}$ & 0.00 & 0.00 & 0.00 & 0.00 & 0.00 & 0.00 & $\mathrm{~F}$ & 0.24 \\
\hline $\mathrm{Cr}$ & 0.00 & 0.00 & 0.00 & 0.00 & 0.00 & 0.00 & $\mathrm{H}_{2} \mathrm{O}$ & 3.00 \\
\hline $\mathrm{Al}$ & 2.30 & 2.35 & 2.26 & 2.24 & 2.26 & 2.25 & Total & 95.52 \\
\hline $\mathrm{Fe}^{2+}$ & 0.00 & 0.00 & 0.00 & 0.00 & 0.00 & 0.00 & (apfu) & \\
\hline $\mathrm{Fe}^{3+}$ & 0.71 & 0.66 & 0.76 & 0.77 & 0.74 & 0.76 & $\mathrm{Si}$ & 3.75 \\
\hline $\mathrm{Mn}$ & 0.00 & 0.01 & 0.00 & 0.00 & 0.00 & 0.00 & $\mathrm{Ti}$ & 0.02 \\
\hline $\mathrm{Mg}$ & 0.00 & 0.00 & 0.00 & 0.00 & 0.00 & 0.00 & $\mathrm{Al}$ & 1.44 \\
\hline $\mathrm{Ca}$ & 1.94 & 1.93 & 2.05 & 2.01 & 2.07 & 2.03 & $\mathrm{Fe}^{3+}$ & 1.05 \\
\hline $\mathrm{Na}$ & 0.00 & 0.00 & 0.00 & 0.00 & 0.00 & 0.00 & $\mathrm{Mn}$ & 0.05 \\
\hline $\mathrm{F}$ & 0.00 & 0.00 & 0.00 & 0.00 & 0.00 & 0.00 & $\mathrm{La}$ & 0.11 \\
\hline \multirow[t]{10}{*}{$\mathrm{Cl}$} & 0.00 & 0.00 & 0.00 & 0.00 & 0.00 & 0.00 & $\mathrm{Ce}$ & 0.18 \\
\hline & & & & & & & $\mathrm{Pr}$ & 0.02 \\
\hline & & & & & & & $\mathrm{Nd}$ & 0.07 \\
\hline & & & & & & & $\mathrm{Sm}$ & 0.01 \\
\hline & & & & & & & $\mathrm{Th}$ & 0.01 \\
\hline & & & & & & & $\mathrm{U}$ & 0.01 \\
\hline & & & & & & & $\mathrm{Ca}$ & 1.28 \\
\hline & & & & & & & $\mathrm{P}$ & 0.00 \\
\hline & & & & & & & $\mathrm{F}$ & 0.07 \\
\hline & & & & & & & $\mathrm{H}$ & 1.93 \\
\hline
\end{tabular}

*recalculated on the basis of Deer et al. (1966, 1972), all Fe was considered as $\mathrm{Fe}^{3+}$

"after grt con." - "after garnet consummation"

43 (Kuklík) exhibit positive $\mathrm{Eu}$ anomalies $(\mathrm{Eu} * / \mathrm{Eu}=$ 2.00-8.62) and low total REE contents.

\subsection{Moldanubian Zone}

The studied samples from nine localities represent a varied set with different mineralogical compositions. The abundances of major- and selected trace elements are given in Tab. 10. Chemistry of the individual samples depends on their modal composition and variability in the composition of the minerals, especially of garnet. Major elements exhibit a wide variation: $20-60$ wt. $\% \mathrm{SiO}_{2}, 1-17$ wt. $\% \mathrm{Al}_{2} \mathrm{O}_{3}, 5-30$ wt. $\% \mathrm{CaO}$, 1-66 wt. $\% \mathrm{Fe}_{2} \mathrm{O}_{3}$ and $0.5-14$ wt. $\% \mathrm{MgO}$. A majority of samples shows a negative correlation between $\mathrm{SiO}_{2}$ and $\mathrm{CaO}$ or $\mathrm{FeO}_{\mathrm{t}}$ (Fig. 7). On the other hand, $\mathrm{Al}_{2} \mathrm{O}_{3}$ correlates positively with $\mathrm{TiO}_{2}, \mathrm{Zr}$ and total REE (Fig. 9). For example, samples from Budeč and Vepřová (Nos 1, 6, 7 and 8), with those from Rešice and Slatina (Nos 14 and 18), contain $\mathrm{Al}_{2} \mathrm{O}_{3}>12$ wt. $\%$ and $\mathrm{TiO}_{2}>0.39$ wt. \%. Their composition is comparable with trends in the trace-element abundances in the paragneisses 
Tab. 9 Whole-rock major- and trace-element compositions from the Svratka Unit. Major oxides in wt. \%, trace elements in ppm, Au in ppb

\begin{tabular}{|c|c|c|c|c|c|c|c|c|c|c|c|c|c|c|c|c|c|c|}
\hline Sample No. & $\mathrm{SiO}_{2}$ & $\mathrm{TiO}_{2}$ & $\mathrm{Al}_{2} \mathrm{O}_{3}$ & $\mathrm{Fe}_{2} \mathrm{O}_{3}$ & MgO & MnO & $\mathrm{CaO}$ & $\mathrm{Na}_{2} \mathrm{O}$ & $\mathrm{K}_{2} \mathrm{O}$ & $\mathbf{P}_{2} \mathbf{O}_{5}$ & Total & $\mathbf{B a}$ & Co & $\mathbf{R b}$ & Sn & $\mathrm{Sr}$ & $\mathbf{Z r}$ & $\mathbf{P b}$ \\
\hline 28 & 41.09 & 0.60 & 17.64 & 3.26 & 2.13 & 0.48 & 21.35 & 0.69 & 0.42 & 0.14 & 99.67 & n.a. & n.a. & 22.2 & 79.1 & 1080.9 & n.a. & 25.1 \\
\hline 29 & 45.00 & 0.40 & 10.58 & 2.47 & 6.56 & 0.63 & 20.65 & 0.58 & 0.14 & 0.14 & 99.80 & n.a. & n.a. & 27.5 & 46.3 & 645.5 & n.a. & 20.4 \\
\hline 30 & 42.63 & 0.37 & 8.37 & 5.84 & 2.95 & 1.41 & 20.83 & 0.33 & 0.08 & 0.14 & 100.40 & n.a. & n.a. & 21.0 & 9.4 & 250.3 & n.a. & 9.6 \\
\hline 32 & 48.26 & 0.07 & 2.46 & 2.00 & 5.21 & 0.42 & 11.61 & 0.66 & 0.03 & 0.08 & 99.23 & 1.2 & 35.1 & 0.5 & 35.0 & 94.4 & 24.4 & 0.3 \\
\hline 34 & 47.92 & 0.11 & 3.76 & 30.04 & 5.05 & 0.59 & 12.16 & 0.62 & 0.04 & 0.12 & 99.89 & 2.0 & 41.9 & 0.6 & 40.0 & 75.8 & 35.3 & 0.6 \\
\hline 35 & 44.81 & 0.62 & 12.32 & 4.50 & 3.87 & 0.28 & 19.77 & 0.99 & 0.66 & 0.09 & 99.86 & n.a. & n.a. & 38.0 & 131.0 & 158.0 & n.a. & 14.0 \\
\hline 36 & 47.28 & 0.61 & 13.43 & 2.92 & 3.39 & 0.30 & 18.90 & 1.86 & 0.41 & 0.14 & 100.01 & n.a. & n.a. & 37.0 & 70.0 & 557.0 & n.a. & 11.0 \\
\hline 37 & 44.82 & 0.36 & 9.64 & 3.58 & 2.24 & 0.42 & 22.28 & 0.28 & 0.35 & 0.16 & 99.90 & n.a. & n.a. & 14.4 & 49.6 & 243.3 & n.a. & 9.8 \\
\hline 38 & 43.63 & 0.55 & 11.53 & 21.60 & 1.96 & 0.35 & 19.53 & 0.47 & 0.26 & 0.14 & 100.02 & n.a. & n.a. & n.a. & n.a. & n.a. & n.a. & n.a. \\
\hline 39 & 42.07 & 0.50 & 11.30 & 23.24 & 1.69 & 0.40 & 19.81 & 0.27 & 0.30 & 0.18 & 99.76 & n.a. & n.a. & n.a. & n.a. & n.a. & n.a. & n.a. \\
\hline 40 & 44.41 & 0.42 & 10.44 & 19.54 & 2.71 & 0.37 & 20.35 & 0.85 & 0.05 & 0.22 & 99.36 & n.a. & n.a. & n.a. & n.a. & n.a. & n.a. & n.a. \\
\hline 41 & 41.78 & 0.54 & 12.39 & 19.44 & 2.16 & 0.36 & 21.72 & 0.79 & 0.05 & 0.18 & 99.79 & 15.0 & 16.4 & 3.5 & 91.0 & 384.7 & 151.2 & 1.1 \\
\hline 42 & 47.00 & 0.02 & 0.88 & 23.94 & 5.60 & 1.60 & 19.86 & 0.38 & 0.01 & 0.09 & 99.79 & 7.0 & 18.7 & 0.8 & 23.0 & 66.2 & 10.0 & 9.7 \\
\hline 43 & 35.29 & 0.02 & 0.91 & 46.84 & 2.69 & 2.05 & 12.18 & 0.18 & 0.08 & 0.07 & 99.78 & 9.0 & 19.6 & 1.8 & 70.0 & 61.1 & 5.0 & 2.5 \\
\hline 44 & 46.30 & 0.31 & 7.58 & 5.78 & 3.11 & 0.44 & 16.92 & 1.89 & 0.11 & 0.09 & 99.17 & n.a. & n.a. & 23.5 & 77.2 & 248.3 & n.a. & 6.7 \\
\hline 45 & 42.76 & 0.56 & 10.88 & 19.79 & 2.45 & 0.37 & 21.58 & 0.94 & 0.08 & 0.18 & 99.78 & 21.0 & 17.3 & 5.2 & 84.0 & 466.1 & 154.9 & 1.1 \\
\hline 47 & 41.28 & 0.58 & 13.00 & 18.77 & 1.97 & 0.41 & 22.93 & 0.13 & 0.02 & 0.18 & 99.72 & 13.0 & 10.0 & 2.6 & 254.0 & 843.5 & 142.2 & 2.6 \\
\hline 48 & 52.26 & 0.57 & 12.51 & 2.30 & 2.94 & 0.41 & 15.87 & 3.89 & 0.30 & 0.14 & 100.19 & 97.8 & 12.0 & 7.5 & 57.0 & 837.1 & 188.0 & 1.4 \\
\hline 49 & 50.20 & 0.49 & 12.18 & 4.24 & 2.45 & 0.48 & 16.94 & 2.62 & 0.23 & 0.14 & 99.80 & 54.0 & 12.5 & 6.2 & 136.0 & 490.7 & 178.1 & 0.8 \\
\hline 50 & 54.58 & 0.39 & 8.55 & 1.86 & 3.01 & 0.61 & 15.20 & 3.17 & 0.20 & 0.16 & 99.35 & 25.6 & 9.7 & 2.7 & 45.0 & 246.8 & 215.0 & 0.7 \\
\hline 51 & 49.47 & 0.65 & 14.56 & 4.34 & 2.15 & 0.36 & 10.53 & 2.12 & 0.62 & 0.18 & 99.83 & 160.2 & 14.2 & 18.0 & 60.0 & 555.5 & 143.4 & 1.7 \\
\hline 52 & 55.06 & 0.41 & 9.93 & 2.29 & 3.36 & 0.29 & 10.82 & 1.25 & 0.47 & 0.13 & 100.23 & 187.1 & 13.7 & 15.6 & 80.0 & 313.0 & 100.4 & 1.5 \\
\hline 53 & 44.94 & 0.39 & 11.35 & 2.28 & 3.59 & 0.40 & 17.48 & 1.58 & 0.49 & 0.14 & 99.47 & 59.3 & 22.8 & 20.9 & 64.0 & 1370.9 & 107.8 & 1.4 \\
\hline 54 & 47.17 & 0.46 & 11.87 & 2.77 & 5.66 & 0.63 & 19.26 & 0.95 & 0.71 & 0.16 & 99.91 & n.a. & n.a. & 42.7 & 84.4 & 486.8 & n.a. & 45.1 \\
\hline 55 & 40.80 & 0.83 & 18.17 & 37.81 & 2.60 & 0.43 & 1.12 & 0.09 & 0.01 & 0.13 & 99.82 & 29.0 & 35.1 & 0.9 & 116.0 & 21.1 & 208.2 & 1.2 \\
\hline 56 & 39.22 & 0.87 & 18.45 & 39.49 & 2.63 & 0.35 & 0.87 & 0.02 & 0.02 & 0.20 & 99.87 & 26.0 & 40.3 & 1.2 & 135.0 & 5.2 & 183.0 & 1.0 \\
\hline 57 & 47.01 & 0.15 & 3.58 & 18.56 & 7.56 & 1.00 & 19.92 & 0.46 & 0.12 & 0.16 & 99.69 & 78.0 & 22.2 & 7.0 & 160.0 & 153.7 & 66.4 & 17.1 \\
\hline
\end{tabular}

\begin{tabular}{|c|c|c|c|c|c|c|c|c|c|c|c|c|c|c|c|c|c|}
\hline ample No. & $\mathbf{Z n}$ & $\mathbf{B i}$ & Au & La & $\mathrm{Ce}$ & Pr & Nd & Sm & Eu & Gd & Tb & Dy & Ho & Er & Tm & $\mathbf{Y b}$ & Lu \\
\hline 28 & 102.1 & n.a. & n.a. & 33.03 & 66.43 & 7.68 & 30.94 & 5.97 & 1.25 & 5.32 & 0.62 & 4.87 & 1.50 & 2.52 & 0.51 & 2.88 & 0.46 \\
\hline 29 & 5.1 & n.a. & n.a. & 22.49 & 43.84 & 5.68 & 19.18 & 4.53 & .77 & 3.77 & n.a. & 3.29 & 86 & 1.39 & 29 & 78 & 0.24 \\
\hline 30 & 8.5 & n.a. & n.a. & 2.14 & 43.52 & 5.36 & 21.26 & 4.23 & .74 & 3.59 & 0.00 & 3.72 & .81 & 1.52 & 32 & 1.67 & 0.23 \\
\hline 32 & 3.0 & 2.2 & 26.9 & 4.40 & 8.00 & 1.02 & 4.30 & 1.00 & 0.30 & 1.15 & 0.17 & 0.97 & 0.17 & 0.61 & .10 & 0.63 & 0.10 \\
\hline 34 & 6.0 & n.a. & & 5.40 & 10.10 & 1.25 & 4.50 & 1.06 & 0.34 & 1.32 & 0.25 & 1.23 & 0.31 & 1.34 & & 2.51 & 0.41 \\
\hline 35 & 48.0 & n.a. & a. & 25.20 & 52.70 & 4.90 & 25.40 & 7.02 & 4.40 & 6.86 & 0.00 & 6.16 & .54 & 2.95 & .40 & 3.68 & 0.52 \\
\hline 36 & 36.0 & n.a. & a. & 38.80 & 72.70 & 7.00 & 29.70 & 4.55 & 2.34 & 5.81 & 0.92 & 5.13 & 1.54 & 3.46 & 0.49 & 3.42 & 0.50 \\
\hline 37 & 57.2 & 5.8 & 0.1 & 54.20 & 94.60 & 9.90 & 43.80 & 8.86 & 1.67 & 6.55 & 1.11 & 5.53 & 1.48 & 2.49 & 0.50 & 3.00 & 0.43 \\
\hline 38 & n.a. & n.a. & n.a. & 62.90 & 108.80 & 11.42 & 38.60 & 5.90 & 64 & 5.05 & 0.81 & 94 & 91 & 2.38 & 35 & .35 & .30 \\
\hline 39 & n.a. & n.a. & n.a. & 31.50 & 56.90 & 6.71 & 26.50 & 5.40 & 1.26 & 4.65 & 0.86 & 4.50 & 0.91 & 2.45 & 0.39 & 2.23 & 0.34 \\
\hline 40 & n.a. & n.a. & a. & 20.60 & 38.80 & 4.88 & 20.30 & 4.20 & 1.42 & 4.53 & 0.90 & 4.56 & 0.98 & 2.84 & 0.43 & 2.32 & 0.43 \\
\hline 41 & 8.0 & 0.8 & 0.5 & 28.80 & 63.10 & 7.28 & 29.60 & 5.15 & 1.20 & 4.68 & 0.81 & 4.39 & 94 & 2.58 & 41 & 2.44 & .34 \\
\hline 42 & 70.0 & 21.4 & 1.4 & 3.80 & 1.70 & 0.34 & 1.10 & 0.11 & 0.18 & 0.18 & 0.04 & 0.29 & 0.06 & 0.14 & 0.03 & 0.14 & 0.02 \\
\hline 43 & 120.0 & 5.0 & 0.8 & 2.40 & 3.00 & 0.45 & 1.90 & 0.45 & 1.35 & 0.51 & 0.08 & 0.46 & 0.10 & 0.28 & .04 & 0.21 & 0.03 \\
\hline 44 & 77.2 & n.a. & n.a. & 7.13 & 20.81 & 3.38 & 14.33 & 3.64 & 0.84 & 3.02 & 0.00 & 2.91 & .52 & 1.59 & .29 & 1.52 & .20 \\
\hline 45 & 8.0 & 0.8 & 0.5 & 35.70 & 71.40 & 8.73 & 34.20 & 5.77 & 1.14 & 5.03 & 0.82 & 4.49 & 0.92 & 2.64 & 0.40 & 2.53 & 0.36 \\
\hline 47 & 23.0 & 0.4 & 0.5 & 37.70 & 70.80 & 9.14 & 35.80 & 6.21 & 1.64 & 5.32 & 0.86 & 4.68 & 0.92 & 2.72 & 0.43 & 2.82 & .40 \\
\hline 48 & 3.0 & 0.1 & 1.2 & 37.00 & 71.00 & 8.27 & 32.90 & 6.80 & 1.62 & 5.99 & 0.94 & 5.01 & 3 & 2.78 & .41 & 2.96 & 0.42 \\
\hline 49 & 7.0 & 0.2 & 0.9 & 38.90 & 69.80 & 8.52 & 33.60 & 6.70 & 4.27 & 6.40 & 1.03 & 5.71 & 1.07 & 3.24 & 0.48 & 2.97 & 0.45 \\
\hline 50 & 3.0 & 0.1 & 0.9 & 28.20 & 54.50 & 6.35 & 25.90 & 4.80 & 1.33 & 3.69 & 0.48 & 2.80 & 47 & 1.28 & 20 & 1.44 & .21 \\
\hline 51 & 15.0 & 0.4 & 1.8 & 50.20 & 80.30 & 11.00 & 44.60 & 7.40 & 2.20 & 5.40 & 0.83 & 4.83 & 0.94 & 2.87 & 0.44 & 3.16 & 0.42 \\
\hline 52 & 15.0 & 0.3 & 19.7 & 25.90 & 39.80 & 6.28 & 25.50 & 5.00 & 1.86 & 4.54 & 0.70 & 3.79 & 0.77 & 2.20 & 0.31 & 1.94 & 0.28 \\
\hline 53 & 8.0 & 2.6 & 2.2 & 28.00 & 49.40 & 6.37 & 26.70 & 5.30 & 1.23 & 4.76 & 0.77 & 4.54 & 0.87 & 2.62 & 0.36 & 2.53 & 0.33 \\
\hline 54 & 8.1 & n.a. & n.a. & 31.38 & 59.04 & 6.45 & 27.7 & 4.41 & 1.41 & 4.38 & 0.89 & 4.67 & 0.82 & 2.63 & 0.29 & 2.46 & 0.35 \\
\hline 55 & 71.0 & 0.1 & 0.5 & 18.00 & 37.90 & 4.93 & 19.70 & 4.12 & 1.42 & 5.90 & 1.05 & 6.29 & 1.55 & 4.70 & 0.79 & 4.95 & 0.70 \\
\hline 56 & 57.0 & 0.1 & 0.5 & 36.10 & 69.90 & 8.74 & 35.60 & 6.06 & 1.21 & 5.61 & 0.90 & 4.72 & 0.94 & 2.74 & 0.46 & 2.87 & 0.43 \\
\hline 57 & 62.0 & 4.5 & 1.8 & 9.60 & 17.10 & 2.18 & 7.20 & 1.51 & 0.93 & 1.32 & 0.23 & 1.26 & 0.30 & 0.84 & 0.15 & 1.02 & 0.14 \\
\hline
\end{tabular}

n.a. $=$ not analyzed 
Tab. 10 Whole-rock major- and trace-element compositions of skarns from the Moldanubian Zone. Major oxides in wt. \%, trace elements in ppm, except for $\mathrm{Au}$ which is in $\mathrm{ppb}$

\begin{tabular}{|c|c|c|c|c|c|c|c|c|c|c|c|c|c|c|c|c|c|c|}
\hline Sample No. & $\mathrm{SiO}_{2}$ & $\mathrm{TiO}_{2}$ & $\mathrm{Al}_{2} \mathrm{O}_{3}$ & $\mathrm{Fe}_{2} \mathrm{O}_{3}$ & MgO & MnO & $\mathrm{CaO}$ & $\mathrm{Na}_{2} \mathrm{O}$ & $\mathrm{K}_{2} \mathrm{O}$ & $\mathbf{P}_{2} \mathrm{O}_{5}$ & Total & $\mathbf{B a}$ & Co & $\mathbf{R b}$ & Sn & Sr & $\mathbf{Z r}$ & $\mathbf{P b}$ \\
\hline 1 & 44.98 & 0.39 & 12.37 & 2.63 & 3.76 & 0.51 & 19.35 & 0.69 & 0.87 & 0.14 & 99.19 & n.a. & n.a. & 129.6 & 567.2 & 459.7 & n.a. & 17.5 \\
\hline 2 & 39.39 & 0.33 & 8.71 & 6.46 & 7.61 & 0.57 & 17.16 & 0.39 & 1.53 & 0.24 & 99.03 & n.a. & n.a. & 150.4 & 550.6 & 128.7 & n.a. & 15.7 \\
\hline 4 & 36.94 & 0.23 & 10.49 & 32.67 & 3.58 & 0.43 & 12.16 & 0.84 & 1.07 & 0.07 & 99.86 & 66.0 & 6.2 & 25.7 & 494.0 & 163.9 & 47.0 & 2.5 \\
\hline 5 & 20.45 & 0.19 & 3.26 & 66.39 & 3.09 & 0.16 & 4.99 & 0.39 & 0.38 & 0.11 & 99.71 & 50.0 & 6.8 & 13.0 & 419.0 & 20.2 & 38.9 & 1.0 \\
\hline 6 & 51.27 & 0.67 & 16.84 & 1.88 & 0.51 & 0.13 & 17.72 & 1.16 & 0.36 & 0.17 & 99.70 & n.a. & n.a. & n.a. & n.a. & n.a. & n.a. & n.a. \\
\hline 7 & 55.77 & 0.60 & 15.29 & 0.66 & 2.48 & 0.15 & 12.90 & 3.02 & 0.96 & 0.19 & 99.54 & n.a. & n.a. & n.a. & n.a. & n.a. & n.a. & n.a. \\
\hline 8 & 50.97 & 0.61 & 16.56 & 1.68 & 0.58 & 0.13 & 18.70 & 0.97 & 0.20 & 0.19 & 97.80 & n.a. & n.a. & n.a. & n.a. & n.a. & n.a. & n.a. \\
\hline 13 & 44.17 & 0.51 & 4.51 & 22.53 & 2.03 & 1.03 & 24.41 & 0.15 & 0.03 & 0.16 & 99.89 & 26.0 & 23.8 & 2.6 & 183.0 & 58.4 & 105.9 & 1.5 \\
\hline 14 & 60.32 & 0.86 & 15.56 & 4.93 & 2.45 & 0.05 & 5.62 & 3.96 & 5.24 & 0.23 & 99.85 & 450.0 & 8.8 & 255.8 & 42.0 & 146.7 & 196.0 & 0.8 \\
\hline 15 & 43.22 & 0.33 & 9.10 & 16.32 & 4.28 & 0.34 & 24.59 & 0.62 & 0.08 & 0.12 & 99.99 & 6.0 & 10.3 & 5.2 & 128.0 & 101.2 & 92.5 & 1.1 \\
\hline 16 & 37.77 & 0.23 & 10.82 & 18.33 & 1.30 & 0.41 & 29.22 & 0.24 & 0.03 & 0.14 & 99.91 & 4.0 & 4.5 & 2.8 & 211.0 & 72.1 & 84.2 & 1.3 \\
\hline 17 & 50.92 & 0.07 & 1.10 & 9.09 & 14.27 & 0.13 & 23.34 & 0.33 & 0.02 & 0.06 & 99.70 & 3.0 & 8.3 & 1.3 & 55.0 & 29.5 & 19.8 & 0.8 \\
\hline 18 & 41.24 & 0.83 & 13.84 & 26.51 & 2.49 & 0.22 & 13.59 & 1.59 & 0.24 & 0.20 & 99.73 & 32.0 & 18.1 & 17.6 & 554.0 & 67.9 & 438.6 & 0.3 \\
\hline 19 & 39.37 & 0.34 & 16.81 & 27.45 & 1.99 & 0.25 & 13.79 & 0.87 & 0.10 & 0.19 & 99.91 & 11.0 & 15.1 & 5.2 & 277.0 & 32.8 & 151.6 & 0.9 \\
\hline 20 & 47.41 & 0.08 & 1.52 & 26.05 & 3.27 & 0.81 & 21.06 & 0.19 & 0.03 & 0.09 & 99.75 & 6.0 & 29.4 & 2.6 & 9.0 & 100.9 & 20.8 & 0.6 \\
\hline 21 & 39.00 & 0.36 & 8.82 & 18.31 & 1.64 & 0.44 & 30.11 & 0.06 & 0.01 & 0.12 & 99.94 & 2.0 & 5.4 & 0.4 & 67.0 & 71.5 & 134.3 & 0.5 \\
\hline 22 & 45.00 & 0.34 & 8.66 & 22.94 & 2.15 & 0.56 & 19.15 & 0.90 & 0.19 & 0.12 & & & 12.3 & 11.5 & 34.0 & 287.3 & & 1.6 \\
\hline 23 & 46.02 & 0.05 & 1.23 & 23.66 & 5.06 & 1.15 & 21.87 & 0.67 & 0.01 & 0.08 & 99.79 & 7.0 & 25.3 & 0.3 & 143.0 & 20.6 & 15.5 & 22.2 \\
\hline 24 & 45.08 & 0.05 & 1.22 & 23.63 & 4.84 & 1.10 & 23.00 & 0.63 & 0.01 & 0.07 & 99.84 & 6.0 & 22.9 & 0.3 & 139.0 & 22.6 & 13.5 & 33.8 \\
\hline 25 & 35.12 & 0.54 & 8.53 & 10.47 & 4.88 & 0.98 & 27.85 & 0.58 & 0.45 & 0.37 & 99.74 & 41.0 & 3.6 & 26.7 & 313.0 & 262.6 & 176.0 & 10.3 \\
\hline 26 & 47.99 & 0.08 & 2.04 & 21.69 & 5.85 & 1.90 & 17.93 & 0.54 & 0.07 & 0.12 & 99.81 & 265.0 & 25.5 & 2.2 & 47.0 & 62.0 & 25.2 & 22.9 \\
\hline 27 & 43.79 & 0.08 & 2.26 & 30.66 & 4.75 & 2.08 & 16.10 & 0.37 & 0.15 & 0.12 & 99.87 & 19.0 & 25.9 & 2.8 & 89.0 & 78.5 & 26.2 & 6.3 \\
\hline Sample No. & $\mathbf{Z n}$ & $\mathbf{B i}$ & Au & $\mathrm{Cu}$ & La & $\mathrm{Ce}$ & Pr & Nd & $\mathrm{Sm}$ & Eu & & Tb & Dy & Ho & Er & $\mathrm{Tm}$ & Yb & Lu \\
\hline 1 & 131.7 & n.a. & n.a. & 16.6 & 40.54 & 81.98 & 9.97 & 35.89 & 7.55 & 2.21 & 6.65 & 0.34 & 7.14 & 1.34 & 3.39 & 0.67 & & 0.57 \\
\hline 2 & 250.2 & n.a. & n.a. & 143.2 & 9.29 & 24.84 & 3.66 & 17.30 & 5.24 & 2.29 & 4.38 & 0.68 & 4.1 & 0.72 & 1.87 & 0.23 & 2.10 & 0.31 \\
\hline 4 & 121.0 & 0.8 & 1.1 & n.a. & 16.80 & 40.50 & 5.51 & 21.30 & 5.08 & 1.80 & 5.27 & 1.10 & 6.38 & 1.32 & 3.82 & 0.69 & 4.46 & 0.64 \\
\hline 5 & 71.0 & 1.8 & 1.8 & n.a. & 7.50 & 15.40 & 2.07 & 8.90 & 1.52 & 0.72 & 1.22 & 0.20 & 1.03 & 0.23 & 0.64 & 0.11 & 0.62 & 0.09 \\
\hline 6 & n.a. & n.a. & n.a. & n.a. & 45.15 & 89.35 & 10.42 & 42.12 & 8.69 & 1.46 & 7.35 & 0.67 & 6.28 & 1.51 & 2.82 & 0.53 & 3.56 & 0.49 \\
\hline 7 & n.a. & n.a. & n.a. & 255.5 & 42.57 & 77.02 & 9.20 & 35.55 & 9.69 & 1.50 & 6.74 & 0.90 & 5.60 & 1.21 & 2.34 & 0.46 & 3.53 & 0.51 \\
\hline 8 & n.a. & n.a. & n.a. & 457.9 & 39.73 & 77.26 & 8.70 & 34.82 & 7.52 & 2.59 & 6.59 & 0.76 & 5.21 & 1.45 & 2.59 & 0.51 & 2.95 & 0.40 \\
\hline 13 & 13.0 & 5.5 & 0.7 & 1.2 & 15.90 & 30.10 & 3.66 & 14.30 & 2.71 & 0.53 & 2.85 & 0.50 & 3.00 & 0.63 & 1.75 & 0.28 & 1.77 & 0.24 \\
\hline 14 & 10.0 & 0.7 & 0.5 & 1.7 & 62.40 & 132.20 & 16.97 & 65.00 & 12.65 & 2.26 & 9.87 & 1.54 & 7.84 & 1.50 & 4.04 & 0.62 & 3.97 & 0.56 \\
\hline 15 & 5.0 & 0.4 & 0.5 & 1.2 & 14.80 & 33.30 & 4.73 & 19.20 & 4.72 & 3.52 & 4.93 & 0.87 & 4.82 & 1.07 & 3.05 & 0.52 & 3.35 & 0.45 \\
\hline 16 & 4.0 & .4 & 0.5 & 0.6 & 10.20 & 20.00 & 3.05 & 15.10 & 4.61 & 4.43 & 5.12 & 0.96 & 5.76 & 1.32 & 4.28 & 0.78 & & 0.62 \\
\hline 17 & 9.0 & 0.8 & 0.5 & 0.8 & 4.90 & 13.10 & 1.83 & 7.40 & 1.58 & 0.41 & 1.30 & 0.20 & 0.95 & 0.20 & 0.49 & 0.07 & 0.52 & 0.07 \\
\hline 18 & 11.0 & 1.3 & 0.5 & 1.0 & 46.00 & 124.60 & 18.63 & 78.00 & 16.29 & 4.65 & 12.70 & 2.43 & 15.06 & 3.29 & 9.77 & 1.65 & 10.79 & 1.39 \\
\hline 19 & 8.0 & 0.8 & 2.8 & 0.4 & 13.70 & 51.30 & 5.74 & 25.40 & 6.19 & 1.96 & 6.53 & 1.46 & 9.75 & 2.30 & 6.65 & 1.11 & 7.32 & 0.92 \\
\hline 20 & 7.0 & 0.2 & 0.5 & 4.1 & 19.50 & 34.20 & 4.44 & 15.00 & 1.62 & 0.39 & 1.11 & 0.12 & 0.63 & 0.10 & 0.27 & n.a. & 0.19 & 0.03 \\
\hline 21 & 10.0 & 0.3 & 3.1 & 1.0 & 6.80 & 14.20 & 2.32 & 14.00 & 3.71 & 0.72 & 3.34 & 0.54 & 3.02 & 0.56 & 1.61 & 0.26 & 1.61 & 0.24 \\
\hline 22 & 9.0 & 0.5 & 1.6 & 1.0 & 20.40 & 42.40 & 5.01 & 19.00 & 3.52 & 0.75 & 3.25 & 0.54 & 2.90 & 0.61 & 1.66 & 0.27 & 1.61 & 0.24 \\
\hline 23 & 90.0 & 1.4 & 3.3 & 2.1 & 3.10 & 6.20 & 0.78 & 3.10 & 0.53 & 0.34 & 0.50 & 0.09 & 0.52 & 0.08 & 0.27 & 0.05 & 0.28 & 0.04 \\
\hline 24 & 72.0 & 2.5 & 0.5 & 2.0 & 2.40 & 4.40 & 0.61 & 2.40 & 0.39 & 0.36 & 0.50 & 0.08 & 0.53 & 0.08 & 0.26 & 0.04 & 0.20 & 0.03 \\
\hline 25 & 139.0 & 9.1 & 24.8 & 210.5 & 22.00 & 45.30 & 5.75 & 22.70 & 5.20 & 1.09 & 5.38 & 0.92 & 5.51 & 1.15 & 3.73 & 0.70 & 4.03 & 0.68 \\
\hline 26 & 38.0 & 126.3 & 6.3 & 0.1 & 6.10 & 10.80 & 1.43 & 5.90 & 1.07 & 0.65 & 0.98 & 0.17 & 1.00 & 0.19 & 0.60 & 0.09 & 0.60 & 0.09 \\
\hline 27 & 76.0 & 29.4 & 4.5 & 1.4 & 6.00 & 12.30 & 1.44 & 6.00 & 1.10 & 1.26 & 1.08 & 0.18 & 0.91 & 0.17 & 0.52 & 0.08 & 0.45 & 0.07 \\
\hline
\end{tabular}

n.a. = not analyzed

of the Varied Group, Moldanubian Zone (Hrdličková ed. 2008). The skarns are characterised by significant variation in the REE contents. The normalized REE patterns resemble those for sediments, $0.2-0.8$ Ga old (Taylor and McLennan 1985; Condie 1993) with typical 100-fold enrichment in LREE, 10-fold enrichment in HREE and a slightly negative Eu anomaly. The LREE/HREE ratio is thus $\sim 10$, which corresponds to the values for post-Archaean sedimentary rocks (Fig. 8).

The Eu/Eu* values span a wide range of 0.5 to 3.5 but the majority of samples are characterised by negligible 
magnitude of the Eu anomaly. The highest $\mathrm{Eu} / \mathrm{Eu}$ * values are typical of samples with low $\Sigma$ REE. Differences in the contents of the major elements, total REE contents and $\mathrm{Eu} / \mathrm{Eu}^{*}$ values occur not only among individual localities but also among samples from the same locality.

Skarns with abundant garnet are enriched in HREE, which results in a rather flat REE distribution pattern. Clinopyroxene skarns from Rešice (sample 17) and Budeč (5), exhibiting the lowest values of $\Sigma$ REE, contain low $\mathrm{Al}_{2} \mathrm{O}_{3}$ and $\mathrm{TiO}_{2}$.

Magnetite-bearing skarn from Rešice contains extremely low $\mathrm{SiO}_{2}$, high $\mathrm{Fe}_{2} \mathrm{O}_{3}$, and somewhat elevated $\mathrm{Cu}$, As, Sn and Zn. Skarn from Budeč (sample 5) shows remarkably low $\mathrm{Zr}, \mathrm{Sr}$ and $\mathrm{Rb}$. All the samples from Budeč and Slatina are enriched in Sn, up to $560 \mathrm{ppm}$.

Skarns from Vlastějovice, Holšice and Zliv (samples 20-25) are split in two groups. The first group features REE abundances resembling detrital sediments (Holšice, Vápenka and Vlastějovice, sample Nos 20-22, 25). Sample 20 exhibits extreme enrichment in LREE and low HREE abundances, combined with low contents of lithophile elements $\mathrm{Rb}, \mathrm{Ba}$ and $\mathrm{K}$. The second group is represented by skarn from Zliv (samples 23 and 24) with extremely low LREE as well as HREE abundances (10× chondrite) and a prominent positive Eu anomaly (Eu/ $\mathrm{Eu}^{*}=2.02-2.49$ ). The two samples have high $\mathrm{Mg}$ and $\mathrm{Ca}$ contents, as well as increased $\mathrm{Co}, \mathrm{Pb}$ and $\mathrm{Zn}$ with low $\mathrm{Al}_{2} \mathrm{O}_{3}, \mathrm{TiO}_{2}$ and $\mathrm{Zr}$ concentrations.

\subsection{Kutná Hora Complex}

Samples from four localities represent mainly Grt$\mathrm{Cpx}$ assemblages with variable contents of the major elements: $22-55$ wt. $\% \mathrm{SiO}_{2}, 2-14$ wt. $\% \mathrm{Al}_{2} \mathrm{O}_{3}, 14-31$ wt. $\% \mathrm{CaO}, 2-58$ wt. $\% \mathrm{Fe}_{2} \mathrm{O}_{3}$ and $0.5-4$ wt. $\% \mathrm{MgO}$. The trace-element abundances are variable for the individual localities as well as for the individual samples (Tab. 11). The samples from Točice and Církvice (Nos 66, 68-69) exhibit REE and other trace-element contents alike post-Archaean sedimentary rocks. The chondritenormalized patterns are also similar to many samples from the Moldanubian Zone and the Svratka Unit, with typical 100-fold enrichment in LREE and 10 fold enrichment in HREE.

Skarns from Malešov (samples 60 and 61), Jakub near Církvice (sample 67) and Církvice (sample 65) have lower contents of REE and other trace elements. The samples from Malešov (Nos 58 and 59) are largely different, with low abundances of REE and absence of enrichment in LREE compared with HREE. All these samples with relatively low REE (Nos 58-59, 61, 65 and 67) have low $\mathrm{Al}_{2} \mathrm{O}_{3}$ and $\mathrm{TiO}_{2}$ contents. Sample 67 has a positive Eu anomaly $\left(\mathrm{Eu} / \mathrm{Eu}^{*}=3.5\right)$.

\section{Oxygen isotopes}

The oxygen isotopic composition in garnets, pyroxenes and amphiboles from skarns of the Svratka Unit is given in Tab. 12. The Fig. 11 indicates that equilibrium was probably reached for most of the co-existing garnetclinopyroxene pairs. The $\delta^{18} \mathrm{O}$ values for garnet and clinopyroxene vary in the interval of -0.1 to $+4.1 \%$. Only the sample SV 297 exhibits isotope disequilibrium between garnet and clinopyroxene.

\section{Geochronological data}

The isotopic ratios obtained from 106 zircon grains from samples SV 25 and SV 262 (Svratouch) are concordant or plot close to the Concordia (Fig. 12, Tab. 13). The $\mathrm{U}-\mathrm{Pb}$ ages for individual zircon grains range broadly from $500 \mathrm{Ma}$ to $2.6 \mathrm{Ga}$ with a marked maximum between 540 and $600 \mathrm{Ma}$, accompanied by minor peaks at $\sim 1.8$ $\mathrm{Ga}, \sim 1.9-2.0 \mathrm{Ga}, \sim 2.45 \mathrm{Ga}$ and $\sim 2.6 \mathrm{Ga}$. Such a span of ages and the presence of marked age maxima are typical of detrital zircon populations and thus indicative of a sedimentary protolith of the studied sample.

\section{Discussion}

\subsection{Metamorphic development}

\subsubsection{The Svratka Unit}

Pressure-temperature development was studied in mica schists and paragneisses in the proximity of skarn bodies (Pertoldová et al. 2007). Structural relations and element partition among minerals indicate three metamorphic stages: $M_{1}$ early prograde, $M_{2}$ prograde/peak and $M_{3}$ retrograde. The metamorphic conditions attained highpressure conditions, corresponding to the kyanite stability field, followed by nearly isothermal decompression characterized by crystallization of sillimanite.

The effects of stage $M_{1}$, characterized by prograde garnet zoning, were observed in all the studied samples. Stage $M_{2}$ is defined on the basis of mineral assemblages with garnet, clinopyroxene, muscovite, biotite, staurolite and kyanite, indicating a metamorphic peak. This stage was characterised by an increase in the P-T conditions straddling the boundary of the amphibolite-facies field to the adjacent lower granulite- and eclogite-facies domains. Phase $M_{3}$ was related to decompression returning back to the amphibolite-facies P-T conditions. Chlorite and sillimanite formed as a result of re-equilibration of the $\mathrm{M}_{1}$ assemblages. Retrogressive sillimanite appears as a reaction product from staurolite or micas in mica schists. 
(a)

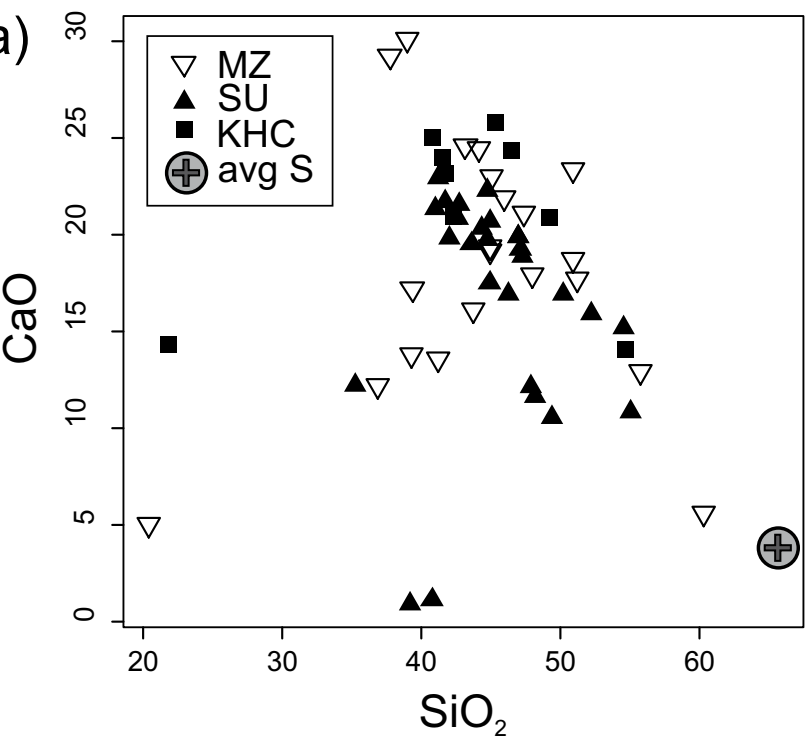

(c)
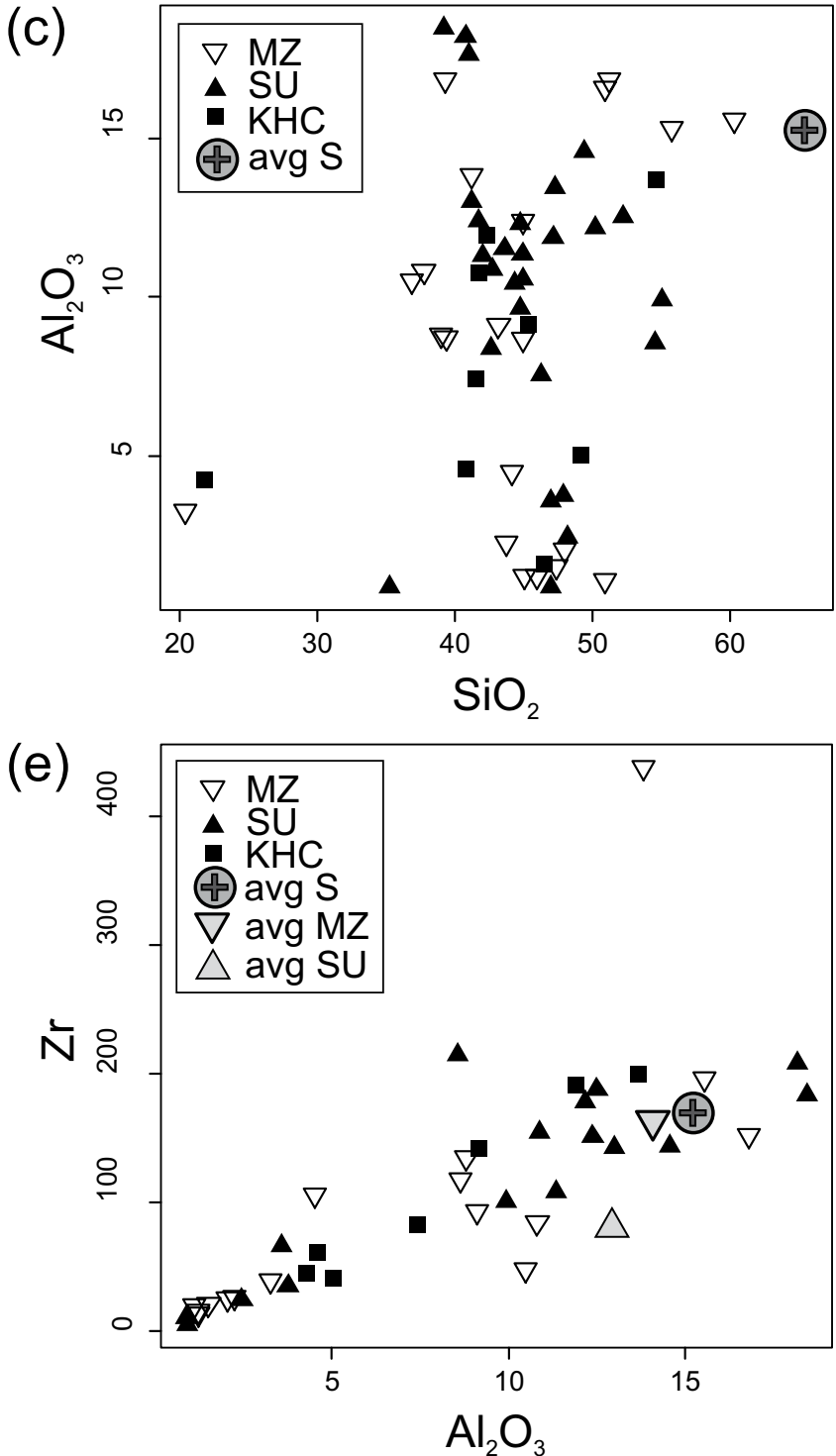
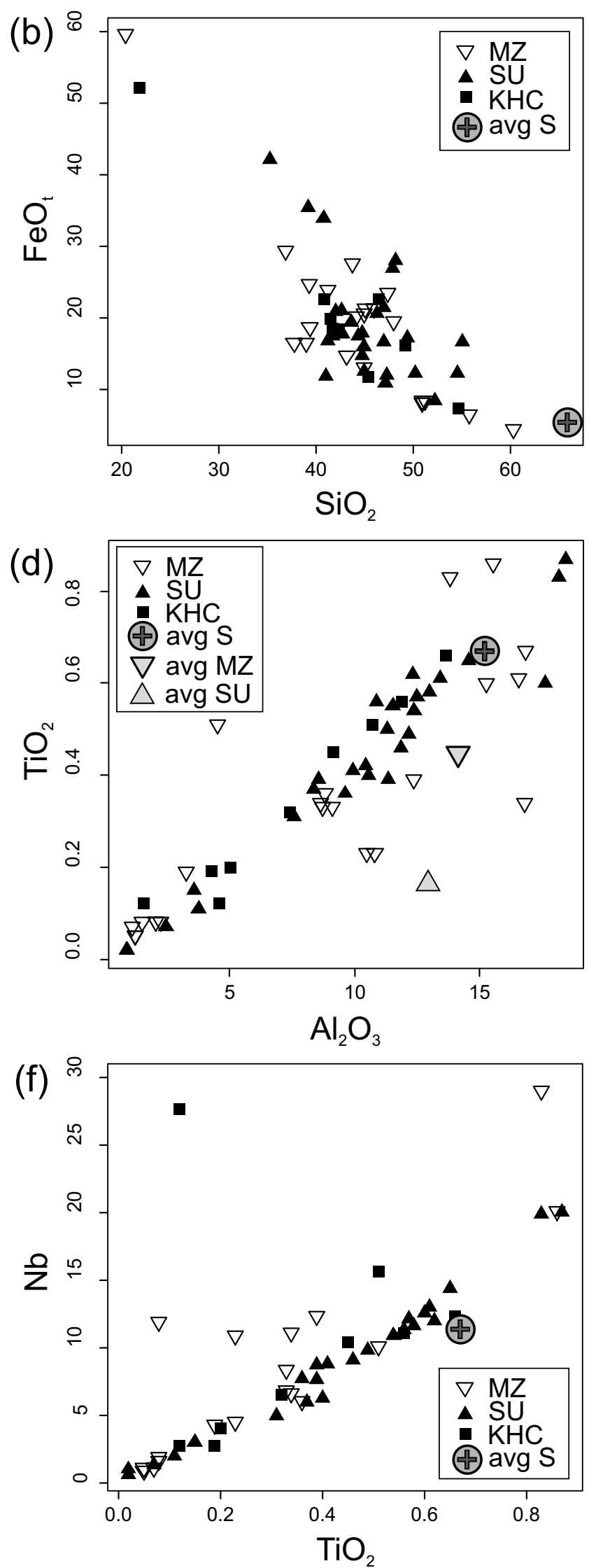
Tab. 11 Whole-rock major- and trace-element compositions of skarns from the Kutná Hora Complex. Major oxides in wt. \%, trace elements in ppm, Au in ppb

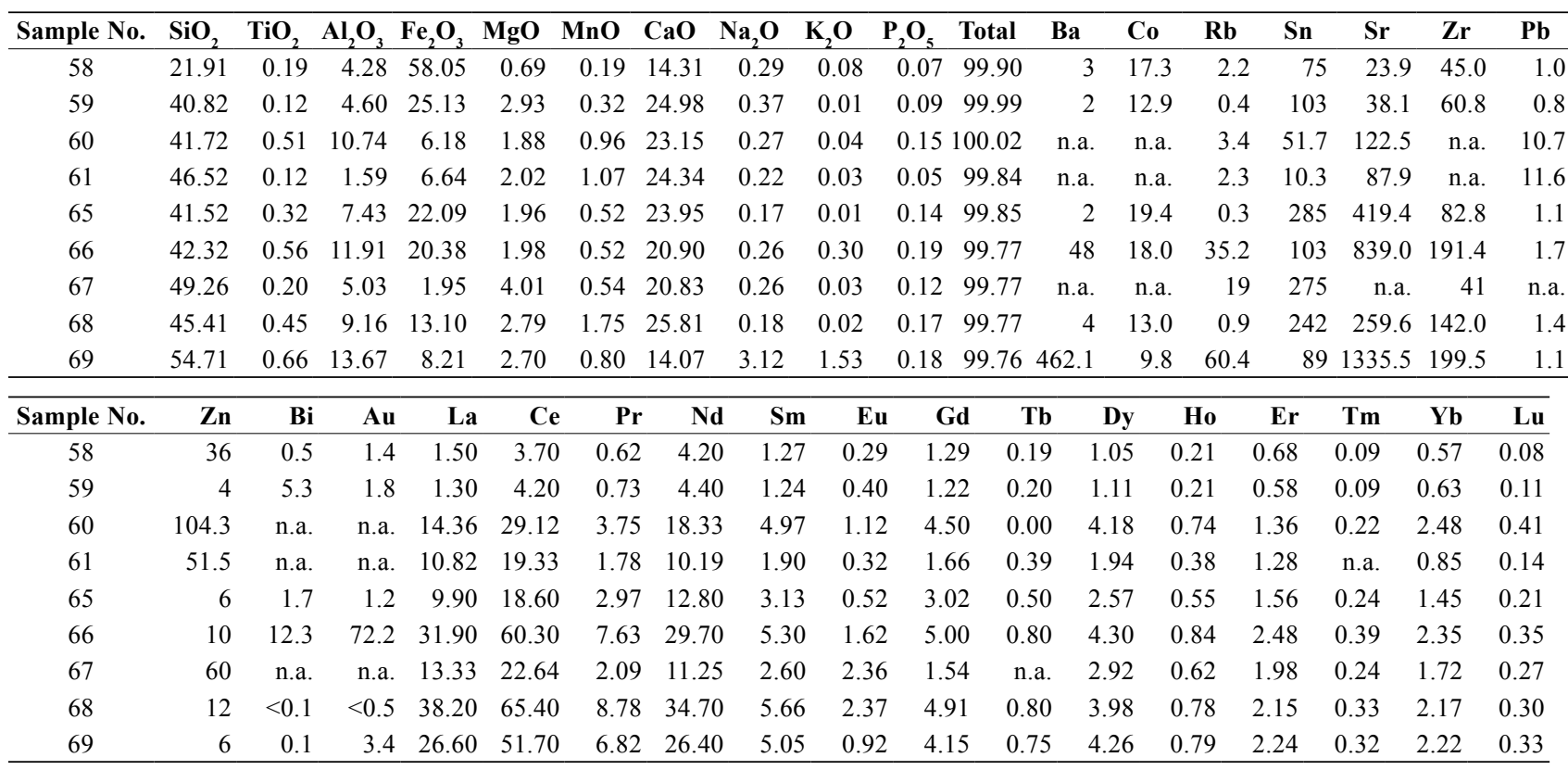

n.a. = not analyzed

Chlorite is a late retrogression mineral, which locally replaced biotite and garnet.

Overall, the prograde P-T path for rocks of the Svratka Unit begun at c. $610^{\circ} \mathrm{C}$ and $0.55 \mathrm{GPa}$, continuing to the peak at $\sim 0.7-0.85 \mathrm{GPa}$ and max. $720^{\circ} \mathrm{C}$ for mica schists, paragneisses and migmatites and up to $1.4 \mathrm{GPa}$ for skarns (see Fig. 13). The retrograde part of the path started with sillimanite and chlorite crystallization in mica schists as well as by symplectite and corona structures growth in skarns.

Our samples with titanite-plagioclase symplectite (titanite II) replacing a Al- and F-bearing titanite (titanite I) are similar to titanites in eclogites from Central China (Carswell et al. 1996). These authors described two types of breakdown reactions for high-Al titanites: 1) ilmenite-amphibole symplectite, 2) symplectite of low$\mathrm{Al}, \mathrm{F}$ titanite with plagioclase. Aluminium and fluorine contents in titanite I in our samples are distinctly lower

Fig. 7 Geochemical variation diagrams for skarns from all three studied units - the Moldanubian zone (MZ), the Svratka Unit (SU) and the Kutná Hora Complex (KHC) exhibit common features. The compositions of average post-Archaean sedimentary rocks (avg S, Taylor and McLennan 1985), average metasediments from the Moldanubian Zone - Strážek Unit (avg MZ, Hrdličková ed. 2008) and average metasediments from the Svratka Unit (avg SU, Hanžl ed. 2008) are plotted for comparison. There is a fair negative correlation between $\mathrm{SiO}_{2}$ and $\mathrm{CaO}, \mathrm{FeO}_{t}$ and positive correlation for $\mathrm{Al}_{2} \mathrm{O}_{3}$ and $\mathrm{TiO}_{2}$. The $\mathrm{Al}_{2} \mathrm{O}_{3}$ contents are not dependent on the $\mathrm{SiO}_{2}$, as is a common feature for sedimentary clastic rocks. The contents of $\mathrm{Zr}$ and $\mathrm{Nb}$ in most skarns are lower than in clastic sediments, as are those of $\mathrm{Al}$ and Ti.



Fig. 8 The REE contents in skarns from all three studied units normalized to chondritic abundances (Boynton 1984). High variability of the REE contents within the individual units can be seen and compared with the REE trend for average post-Archaean sediments. The progressive fractionation of LREE and increasing magnitude of the positive Eu anomaly are characteristic for some of the studied skarns, resembling the trend known from hydrothermal rift-related deposits on the sea floor (e.g. Hongo et al. 2007). A pattern typical of skarns with prevailing clastic sedimentary content is depicted on an example from Veprová (No. 13), a lower content of detritic material is demonstrated by the REE trend of Kuklík (42) and the influence of hydrothermal activity on the REE composition is characteristic of the Malešov (59) sample. 



Fig. 9 Geochemical variation diagrams $\mathrm{Al}_{2} \mathrm{O}_{3}$ vs. $\Sigma \mathrm{REE}$ and $\mathrm{TiO}_{2}$ vs. $\Sigma \mathrm{REE}$ for the skarns from the Moldanubian Zone (MZ) and the Svratka Unit (SU).

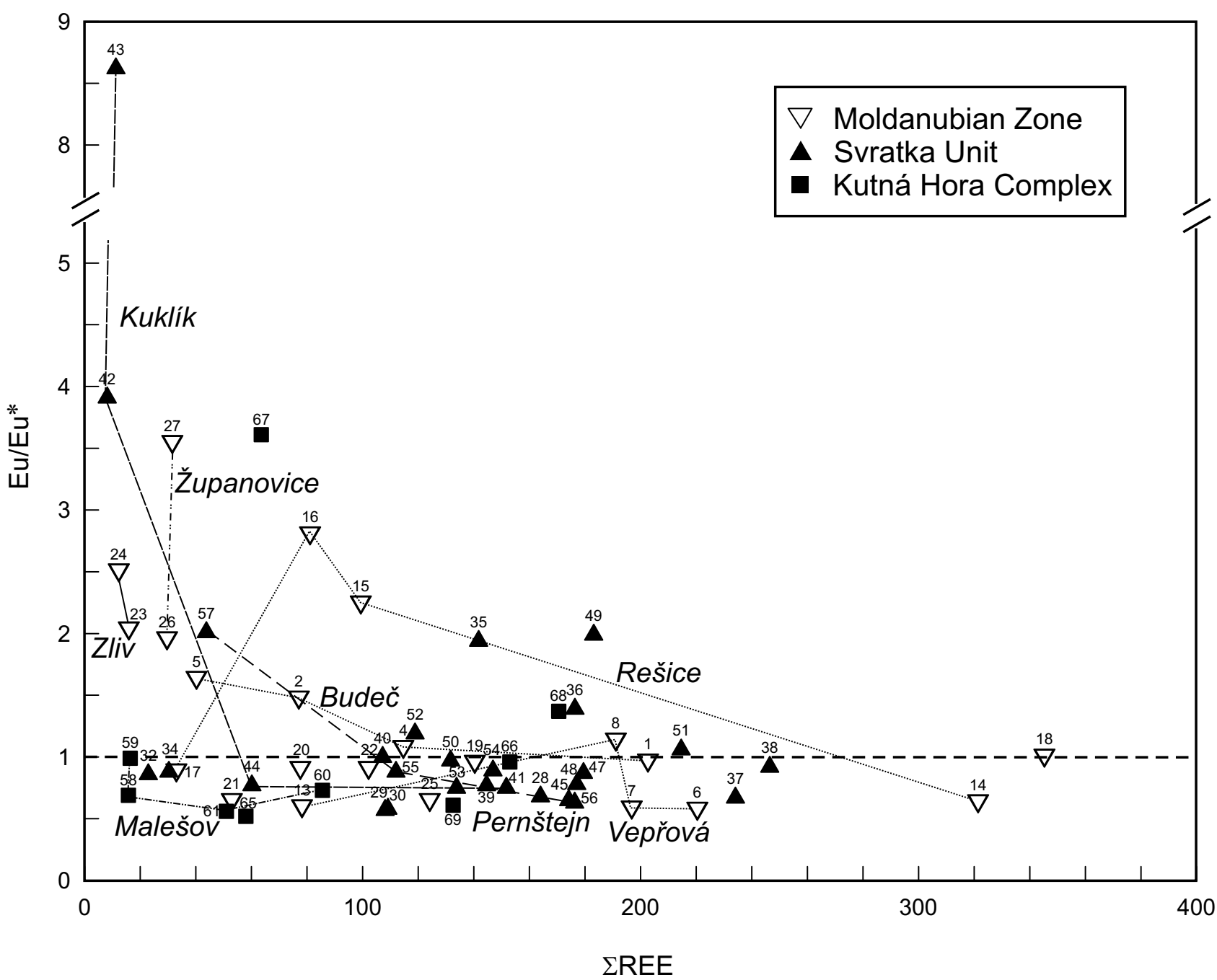

Fig. 10 The diagram of Eu anomaly (Eu/Eu*) vs. $\Sigma$ REE after normalization to chondrites (Boynton 1984). A positive Eu anomaly is characteristic for products of hydrothermal activity near submarine hydrothermal veins. The lower the hydrothermal activity and the lower temperature of circulating fluids, the lesser is their influence on the composition of the surrounding sediments (Chavagnac et al. 2005). Even the skarn samples from the same locality show large variations in their REE contents and patterns, suggesting that the influence of hydrothermal fluids was very local. 


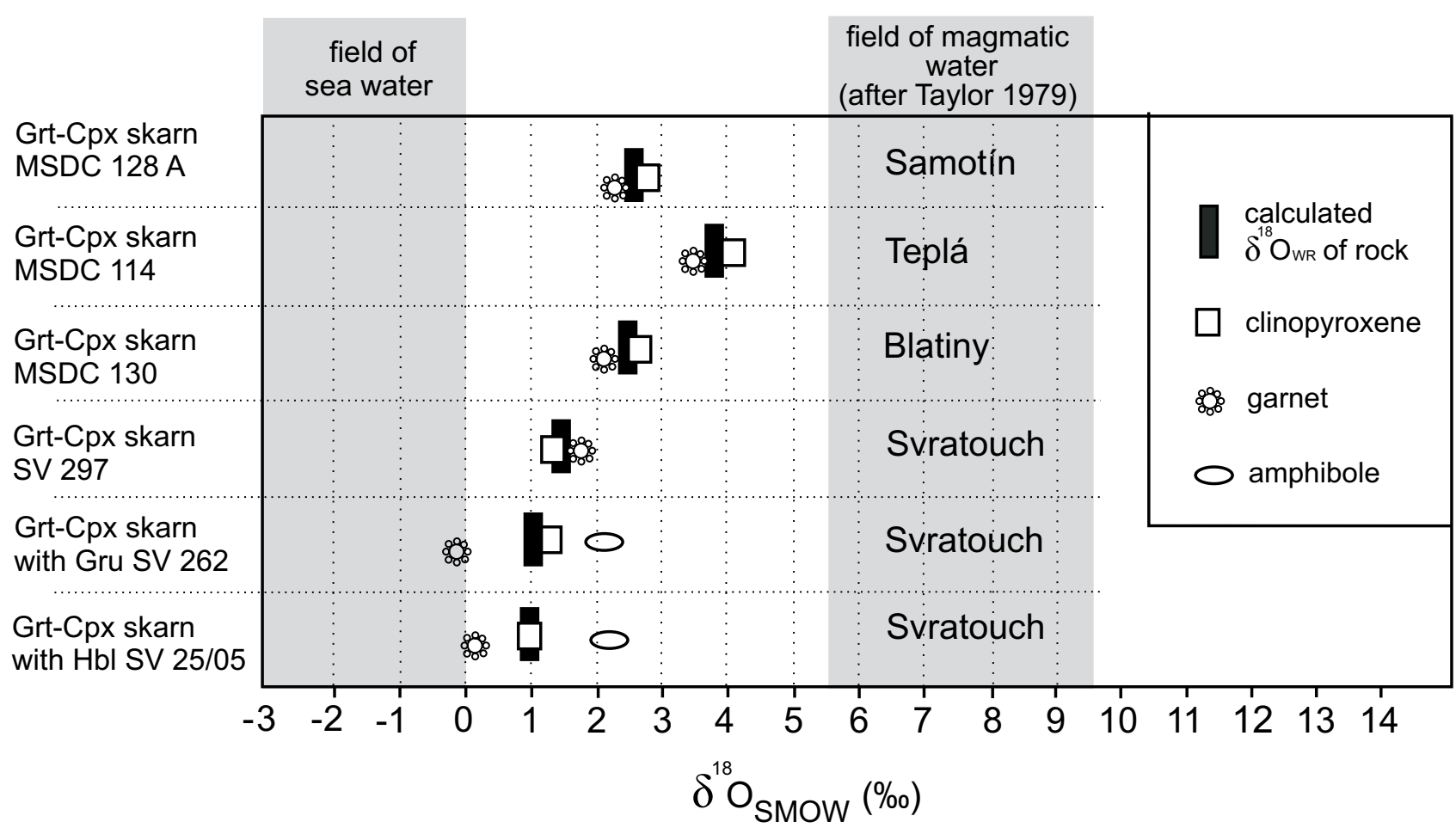

Fig. 11 The $\delta^{18} \mathrm{O}_{\text {SMow }}(\%$ ) values for garnet, clinopyroxene, grunerite and hornblende from skarns (the Svratka Unit). Sea water after Hoefs (2009).

Tab. 12 Oxygen isotope composition expressed as $\delta^{18} \mathrm{O} \%$ (SMOW) for skarn minerals from the Svratka Unit

\begin{tabular}{cccccc}
\hline locality & sample & $\boldsymbol{\delta}^{\mathbf{1 8}} \mathbf{O} \% \mathbf{C p x}$ & $\boldsymbol{\delta}^{\mathbf{1 8}} \mathbf{O} \% \mathbf{G r t}$ & $\boldsymbol{\delta}^{\mathbf{1 8} \mathbf{O} \% \mathbf{0}}$ & $\boldsymbol{\delta}^{\mathbf{1 8}} \mathbf{O}_{\mathbf{0}} \mathbf{\text { Gr }}$ \\
\cline { 5 - 6 } & & & & Hbl, Gru & calculated \\
\hline Samotín & Grt-Cpx skarn MSDC128A & 2.80 & 2.30 & & 2.58 \\
Teplá & Grt-Cpx skarn MSDC114 & 4.10 & 3.50 & & 3.80 \\
Blatiny & Grt-Cpx skarn MSDC130 & 2.70 & 2.10 & & 2.44 \\
Svratouch & Grt-Cpx skarn SV 297 & 1.30 & 1.80 & & 1.46 \\
Svratouch & Grt-Cpx skarn with Gru SV262 & 1.30 & -0.10 & 2.10 & 1.05 \\
Svratouch & Grt-Cpx skarn with Hbl SV25 & 1.00 & 0.10 & 2.20 & 0.86 \\
\hline
\end{tabular}

Tab. 13 Analytical data obtained by LA ICP-MS U-Pb zircon dating

\begin{tabular}{|c|c|c|c|c|c|c|c|c|c|c|c|c|c|c|}
\hline \multirow{2}{*}{$\begin{array}{c}\text { Analysis } \\
\#\end{array}$} & \multicolumn{6}{|c|}{ Isotopic ratios } & \multicolumn{8}{|c|}{ Calculated ages (Ma) } \\
\hline & ${ }^{207} \mathrm{~Pb} /{ }^{235} \mathrm{U}$ & $\pm 1 \sigma$ & ${ }^{206} \mathrm{~Pb} /{ }^{238} \mathrm{U}$ & $\pm 1 \sigma$ & ${ }^{207} \mathrm{~Pb} /{ }^{206} \mathrm{~Pb}$ & $\pm 1 \sigma$ & ${ }^{207} \mathrm{~Pb} /{ }^{235} \mathrm{U}$ & $\pm 1 \sigma$ & ${ }^{206} \mathrm{~Pb} /{ }^{238} \mathrm{U}$ & $\pm 1 \sigma^{2}$ & ${ }^{07} \mathrm{~Pb} /{ }^{206} \mathrm{~Pb}$ & $\pm 1 \sigma$ & Concordia & $\pm 1 \sigma$ \\
\hline 1 & 0.8197 & 0.0360 & 0.1014 & 0.0024 & 0.0586 & 0.0020 & 608 & 20 & 623 & 14 & 554 & 73 & 618 & 13 \\
\hline 2 & 0.7605 & 0.0357 & 0.0901 & 0.0018 & 0.0612 & 0.0025 & 574 & 21 & 556 & 11 & 648 & 88 & 559 & 10 \\
\hline 3 & 0.7896 & 0.0364 & 0.0904 & 0.0018 & 0.0633 & 0.0027 & 591 & 21 & 558 & 10 & 719 & 91 & 562 & 10 \\
\hline 4 & 0.6923 & 0.0223 & 0.0890 & 0.0023 & 0.0564 & 0.0013 & 534 & 13 & 550 & 14 & 469 & 52 & 542 & 11 \\
\hline 5 & 0.6068 & 0.0279 & 0.0851 & 0.0026 & 0.0517 & 0.0014 & 482 & 18 & 526 & 15 & 274 & 61 & 508 & 13 \\
\hline 6 & 0.7030 & 0.0368 & 0.0952 & 0.0020 & 0.0536 & 0.0021 & 541 & 22 & 586 & 12 & 353 & 89 & 578 & 11 \\
\hline 7 & 6.2488 & 0.1607 & 0.3648 & 0.0064 & 0.1242 & 0.0025 & 2011 & 23 & 2005 & 30 & 2018 & 36 & 2009 & 21 \\
\hline 8 & 3.3171 & 0.1711 & 0.2461 & 0.0092 & 0.0978 & 0.0034 & 1485 & 40 & 1418 & 47 & 1582 & 65 & 1459 & 36 \\
\hline 9 & 0.6511 & 0.0443 & 0.0903 & 0.0028 & 0.0523 & 0.0026 & 509 & 27 & 557 & 17 & 298 & 115 & 547 & 15 \\
\hline 10 & 1.5603 & 0.0531 & 0.1706 & 0.0034 & 0.0663 & 0.0016 & 955 & 21 & 1016 & 19 & 816 & 51 & 988 & 16 \\
\hline 11 & 0.7164 & 0.0227 & 0.0883 & 0.0019 & 0.0588 & 0.0016 & 549 & 13 & 546 & 11 & 560 & 60 & 547 & 10 \\
\hline 12 & 11.9667 & 0.3294 & 0.4833 & 0.0118 & 0.1796 & 0.0030 & 2602 & 26 & 2542 & 51 & 2649 & 27 & 2599 & 26 \\
\hline 13 & 0.7583 & 0.0383 & 0.0980 & 0.0026 & 0.0561 & 0.0025 & 573 & 22 & 603 & 16 & 457 & 99 & 594 & 14 \\
\hline 14 & 0.7204 & 0.0410 & 0.0892 & 0.0020 & 0.0585 & 0.0027 & 551 & 24 & 551 & 12 & 550 & 103 & 551 & 11 \\
\hline
\end{tabular}


Tab. 13 continued

\begin{tabular}{|c|c|c|c|c|c|c|c|c|c|c|c|c|c|c|}
\hline \multirow{2}{*}{$\begin{array}{c}\text { Analysis } \\
\# \\
\end{array}$} & \multicolumn{6}{|c|}{ Isotopic ratios } & \multicolumn{8}{|c|}{ Calculated ages (Ma) } \\
\hline & ${ }^{207} \mathrm{~Pb} /{ }^{235} \mathbf{U}$ & $\pm 1 \sigma$ & ${ }^{206} \mathrm{~Pb} /{ }^{238} \mathrm{U}$ & $\pm 1 \sigma$ & ${ }^{207} \mathrm{~Pb} /{ }^{206} \mathrm{~Pb}$ & $\pm 1 \sigma$ & ${ }^{207} \mathrm{~Pb} /{ }^{235} \mathrm{U}$ & $\pm 1 \sigma$ & ${ }^{206} \mathrm{~Pb} /{ }^{238} \mathrm{U}$ & $\pm 1 \sigma^{2}$ & ${ }^{07} \mathrm{~Pb} / 206 \mathrm{~Pb}$ & $\pm 1 \sigma$ & Concordia & $\pm 1 \sigma$ \\
\hline 15 & 0.7255 & 0.0157 & 0.0913 & 0.0016 & 0.0576 & 0.0008 & 554 & 9 & 563 & 9 & 516 & 31 & 559 & 8 \\
\hline 16 & 0.7073 & 0.0242 & 0.0908 & 0.0017 & 0.0565 & 0.0015 & 543 & 14 & 560 & 10 & 473 & 58 & 555 & 9 \\
\hline 17 & 0.7626 & 0.0317 & 0.0954 & 0.0023 & 0.0580 & 0.0019 & 575 & 18 & 588 & 13 & 528 & 71 & 584 & 12 \\
\hline 18 & 0.6070 & 0.0434 & 0.0828 & 0.0017 & 0.0532 & 0.0034 & 482 & 27 & 513 & 10 & 337 & 144 & 510 & 10 \\
\hline 19 & 0.7874 & 0.0381 & 0.0911 & 0.0015 & 0.0627 & 0.0029 & 590 & 22 & 562 & 9 & 699 & 98 & 564 & 9 \\
\hline 20 & 0.6782 & 0.0315 & 0.0850 & 0.0019 & 0.0579 & 0.0025 & 526 & 19 & 526 & 11 & 525 & 96 & 526 & 10 \\
\hline 21 & 4.6722 & 0.1532 & 0.3112 & 0.0082 & 0.1089 & 0.0014 & 1762 & 27 & 1747 & 40 & 1781 & 23 & 1759 & 26 \\
\hline 22 & 0.7210 & 0.0317 & 0.0929 & 0.0027 & 0.0563 & 0.0018 & 551 & 19 & 573 & 16 & 463 & 73 & 565 & 14 \\
\hline 23 & 0.7070 & 0.0218 & 0.0888 & 0.0022 & 0.0577 & 0.0017 & 543 & 13 & 549 & 13 & 520 & 65 & 546 & 11 \\
\hline 24 & 5.5354 & 0.1738 & 0.3347 & 0.0098 & 0.1200 & 0.0018 & 1906 & 27 & 1861 & 47 & 1956 & 27 & 1902 & 27 \\
\hline 25 & 0.6449 & 0.0265 & 0.0864 & 0.0016 & 0.0541 & 0.0020 & 505 & 16 & 534 & 10 & 376 & 82 & 528 & 9 \\
\hline 26 & 5.8659 & 0.1864 & 0.3394 & 0.0106 & 0.1254 & 0.0025 & 1956 & 28 & 1884 & 51 & 2034 & 35 & 1953 & 28 \\
\hline 27 & 0.8466 & 0.0346 & 0.1058 & 0.0027 & 0.0581 & 0.0019 & 623 & 19 & 648 & 16 & 532 & 70 & 639 & 14 \\
\hline 28 & 0.7012 & 0.0647 & 0.0955 & 0.0037 & 0.0532 & 0.0043 & 539 & 39 & 588 & 22 & 338 & 183 & 578 & 20 \\
\hline 29 & 0.7538 & 0.0287 & 0.0951 & 0.0019 & 0.0575 & 0.0018 & 570 & 17 & 586 & 11 & 510 & 70 & 582 & 10 \\
\hline 30 & 0.7226 & 0.0404 & 0.0933 & 0.0027 & 0.0562 & 0.0024 & 552 & 24 & 575 & 16 & 458 & 94 & 569 & 15 \\
\hline 31 & 0.7411 & 0.0306 & 0.0940 & 0.0018 & 0.0572 & 0.0018 & 563 & 18 & 579 & 11 & 499 & 69 & 576 & 10 \\
\hline 32 & 4.7553 & 0.1118 & 0.3069 & 0.0067 & 0.1124 & 0.0016 & 1777 & 20 & 1725 & 33 & 1838 & 26 & 1772 & 20 \\
\hline 33 & 0.6957 & 0.0416 & 0.0968 & 0.0023 & 0.0521 & 0.0024 & 536 & 25 & 596 & 13 & 291 & 107 & 585 & 13 \\
\hline 34 & 1.1507 & 0.0665 & 0.1356 & 0.0039 & 0.0616 & 0.0025 & 778 & 31 & 820 & 22 & 659 & 88 & 807 & 20 \\
\hline 35 & 0.7174 & 0.0425 & 0.0917 & 0.0024 & 0.0568 & 0.0026 & 549 & 25 & 565 & 14 & 483 & 101 & 562 & 13 \\
\hline 36 & 0.6744 & 0.0250 & 0.0899 & 0.0020 & 0.0544 & 0.0016 & 523 & 15 & 555 & 12 & 387 & 68 & 544 & 10 \\
\hline 37 & 0.6446 & 0.0257 & 0.0870 & 0.0019 & 0.0537 & 0.0018 & 505 & 16 & 538 & 11 & 360 & 75 & 528 & 10 \\
\hline 38 & 0.6656 & 0.0227 & 0.0905 & 0.0017 & 0.0533 & 0.0017 & 518 & 14 & 559 & 10 & 343 & 70 & 546 & 9 \\
\hline 39 & 4.5514 & 0.1145 & 0.3145 & 0.0065 & 0.1050 & 0.0016 & 1740 & 21 & 1763 & 32 & 1714 & 27 & 1744 & 20 \\
\hline 40 & 5.8817 & 0.1398 & 0.3671 & 0.0086 & & 0.0022 & 1959 & 21 & 2016 & 40 & 1899 & 34 & 1959 & 21 \\
\hline 41 & 5.9880 & 0.1655 & 0.3596 & 0.0087 & 0.1208 & 0.0025 & 1974 & 24 & 1980 & 41 & 1968 & 37 & 1975 & 24 \\
\hline 42 & 0.7416 & 0.0293 & 0.0972 & 0.0026 & 0.0554 & 0.0019 & 563 & 17 & 598 & 15 & 427 & 75 & 583 & 13 \\
\hline 43 & 5.6645 & 0.1743 & 0.3452 & 0.0093 & 0.1190 & 0.0019 & 1926 & 27 & 1912 & 44 & 1941 & 29 & 1924 & 26 \\
\hline 44 & 0.7047 & 0.0412 & & 0.0029 & & & 542 & 25 & 561 & 17 & 462 & 104 & 555 & 16 \\
\hline 45 & 0.6666 & 0.0415 & 0.0914 & 0.0025 & 0.0529 & 0.0027 & 519 & 25 & 564 & 15 & 324 & 117 & 554 & 14 \\
\hline 46 & 4.8740 & 0.2954 & 0.3362 & 0.0101 & 0.1051 & 0.0044 & 1798 & 51 & 1869 & 49 & 1717 & 77 & 1834 & 39 \\
\hline 47 & 0.6743 & 0.0232 & 0.0911 & 0.0020 & 0.0537 & 0.0015 & 523 & 14 & 562 & 12 & 359 & 62 & 547 & 10 \\
\hline 48 & 0.6685 & 0.0223 & 0.0880 & 0.0022 & 0.0551 & 0.0012 & 520 & 14 & 544 & 13 & 417 & 50 & 533 & 11 \\
\hline 49 & 0.6972 & 0.0226 & 0.0883 & 0.0019 & 0.0573 & 0.0015 & 537 & 13 & 545 & 11 & 502 & 59 & 542 & 10 \\
\hline 50 & 0.7999 & 0.0451 & 0.0932 & 0.0023 & 0.0622 & 0.0034 & 597 & 25 & 575 & 13 & 682 & 116 & 578 & 13 \\
\hline 51 & 0.6673 & 0.0316 & 0.0897 & 0.0023 & 0.0539 & 0.0024 & 519 & 19 & 554 & 14 & 369 & 100 & 544 & 12 \\
\hline 52 & 0.6611 & 0.0217 & 0.0903 & 0.0022 & 0.0531 & 0.0014 & 515 & 13 & 557 & 13 & 333 & 59 & 536 & 11 \\
\hline 53 & 0.6647 & 0.0368 & 0.0879 & 0.0024 & 0.0548 & 0.0023 & 517 & 22 & 543 & 14 & 405 & 92 & 537 & 13 \\
\hline 54 & 0.6660 & 0.0568 & 0.0883 & 0.0025 & 0.0547 & 0.0034 & 518 & 35 & 545 & 15 & 401 & 139 & 542 & 14 \\
\hline 55 & 0.7281 & 0.0203 & 0.0988 & 0.0025 & 0.0534 & 0.0009 & 555 & 12 & 607 & 15 & 348 & 40 & 571 & 11 \\
\hline 56 & 1.1501 & 0.0886 & 0.1248 & 0.0062 & 0.0668 & 0.0023 & 777 & 42 & 758 & 35 & 832 & 73 & 765 & 31 \\
\hline 57 & 4.5589 & 0.1307 & 0.3117 & 0.0080 & 0.1061 & 0.0017 & 1742 & 24 & 1749 & 39 & 1733 & 30 & 1743 & 23 \\
\hline 58 & 10.6351 & 0.2784 & 0.4569 & 0.0124 & 0.1688 & 0.0022 & 2492 & 24 & 2426 & 55 & 2546 & 21 & 2495 & 24 \\
\hline 59 & 0.6522 & 0.0278 & 0.0829 & 0.0024 & 0.0570 & 0.0020 & 510 & 17 & 514 & 14 & 493 & 78 & 512 & 12 \\
\hline 60 & 5.5391 & 0.2076 & 0.3448 & 0.0104 & 0.1165 & 0.0018 & 1907 & 32 & 1910 & 50 & 1903 & 28 & 1907 & 31 \\
\hline 61 & 9.8736 & 0.1911 & 0.4525 & 0.0078 & 0.1582 & 0.0018 & 2423 & 18 & 2406 & 34 & 2437 & 19 & 2422 & 18 \\
\hline 62 & 0.6906 & 0.0396 & 0.0874 & 0.0023 & 0.0573 & 0.0029 & 533 & 24 & 540 & 14 & 503 & 110 & 539 & 13 \\
\hline 63 & 5.7865 & 0.1279 & 0.3652 & 0.0054 & 0.1149 & 0.0020 & 1944 & 19 & 2007 & 26 & 1878 & 31 & 1962 & 17 \\
\hline 64 & 0.8394 & 0.0408 & 0.0975 & 0.0018 & 0.0625 & 0.0029 & 619 & 23 & 600 & 11 & 690 & 98 & 602 & 10 \\
\hline 65 & 0.7408 & 0.0255 & 0.0931 & 0.0019 & 0.0577 & 0.0011 & 563 & 15 & 574 & 11 & 520 & 42 & 570 & 10 \\
\hline 66 & 0.7971 & 0.0277 & 0.0945 & 0.0019 & 0.0612 & 0.0018 & 595 & 16 & 582 & 11 & 646 & 65 & 585 & 10 \\
\hline
\end{tabular}


Tab. 13 continued

\begin{tabular}{|c|c|c|c|c|c|c|c|c|c|c|c|c|c|c|}
\hline \multirow{2}{*}{$\begin{array}{c}\text { Analysis } \\
\#\end{array}$} & \multicolumn{6}{|c|}{ Isotopic ratios } & \multicolumn{8}{|c|}{ Calculated ages (Ma) } \\
\hline & ${ }^{207} \mathrm{~Pb} /{ }^{235} \mathrm{U}$ & $\pm 1 \sigma$ & ${ }^{206} \mathrm{~Pb} /{ }^{238} \mathrm{U}$ & $\pm 1 \sigma$ & ${ }^{207} \mathrm{~Pb} /{ }^{206} \mathrm{~Pb}$ & $\pm 1 \sigma$ & ${ }^{207} \mathrm{~Pb} /{ }^{235} \mathrm{U}$ & $\pm 1 \sigma$ & ${ }^{206} \mathrm{~Pb} /{ }^{238} \mathrm{U}$ & $\pm 1 \sigma^{2}$ & ${ }^{207} \mathrm{~Pb} /{ }^{206} \mathrm{~Pb}$ & $\pm 1 \sigma$ & Concordia & $\pm 1 \sigma$ \\
\hline 67 & 1.1928 & 0.0545 & 0.1377 & 0.0032 & 0.0628 & 0.0017 & 797 & 25 & 832 & 18 & 702 & 56 & 822 & 16 \\
\hline 69 & 0.6740 & 0.0229 & 0.0839 & 0.0021 & 0.0583 & 0.0011 & 523 & 14 & 519 & 12 & 541 & 41 & 521 & 11 \\
\hline 70 & 12.3879 & 0.3170 & 0.4917 & 0.0109 & 0.1827 & 0.0023 & 2634 & 24 & 2578 & 47 & 2678 & 20 & 2631 & 24 \\
\hline 71 & 0.8161 & 0.0302 & 0.0963 & 0.0023 & 0.0614 & 0.0020 & 606 & 17 & 593 & 14 & 655 & 71 & 597 & 12 \\
\hline 72 & 0.6319 & 0.0226 & 0.0823 & 0.0019 & 0.0557 & 0.0014 & 497 & 14 & 510 & 11 & 441 & 57 & 505 & 10 \\
\hline 73 & 0.6717 & 0.0129 & 0.0869 & 0.0011 & 0.0561 & 0.0009 & 522 & 8 & 537 & 6 & 455 & 36 & 532 & 6 \\
\hline 74 & 0.7357 & 0.0226 & 0.0928 & 0.0014 & 0.0575 & 0.0016 & 560 & 13 & 572 & 8 & 511 & 60 & 569 & 8 \\
\hline 75 & 4.9088 & 0.2308 & 0.3292 & 0.0098 & 0.1081 & 0.0021 & 1804 & 40 & 1834 & 48 & 1768 & 35 & 1815 & 35 \\
\hline 76 & 0.7453 & 0.0204 & 0.0905 & 0.0014 & 0.0598 & 0.0013 & 565 & 12 & 558 & 8 & 595 & 48 & 560 & 8 \\
\hline 77 & 0.7294 & 0.0321 & 0.0920 & 0.0013 & 0.0575 & 0.0020 & 556 & 19 & 567 & 8 & 512 & 77 & 566 & 8 \\
\hline 79 & 6.4373 & 0.1658 & 0.3690 & 0.0081 & 0.1265 & 0.0020 & 2037 & 23 & 2025 & 38 & 2050 & 28 & 2036 & 22 \\
\hline 80 & 0.7626 & 0.0365 & 0.0959 & 0.0028 & 0.0577 & 0.0016 & 575 & 21 & 590 & 16 & 517 & 62 & 585 & 15 \\
\hline 81 & 0.7845 & 0.0277 & 0.0960 & 0.0022 & 0.0593 & 0.0013 & 588 & 16 & 591 & 13 & 577 & 48 & 590 & 11 \\
\hline 82 & 0.7002 & 0.0181 & 0.0911 & 0.0014 & 0.0557 & 0.0013 & 539 & 11 & 562 & 8 & 442 & 53 & 555 & 7 \\
\hline 83 & 0.8703 & 0.0239 & 0.1039 & 0.0015 & 0.0607 & 0.0016 & 636 & 13 & 637 & 9 & 630 & 59 & 637 & 8 \\
\hline 84 & 0.6627 & 0.0224 & 0.0838 & 0.0019 & 0.0573 & 0.0014 & 516 & 14 & 519 & 12 & 504 & 54 & 518 & 10 \\
\hline 85 & 0.7478 & 0.0239 & 0.0934 & 0.0015 & 0.0581 & 0.0016 & 567 & 14 & 575 & 9 & 533 & 60 & 573 & 8 \\
\hline 86 & 11.8982 & 0.4510 & 0.4895 & 0.0144 & 0.1763 & 0.0032 & 2596 & 36 & 2568 & 62 & 2618 & 30 & 2593 & 35 \\
\hline 87 & 0.7416 & 0.0217 & 0.0921 & 0.0016 & 0.0584 & 0.0014 & 563 & 13 & 568 & 9 & 545 & 51 & 567 & 8 \\
\hline 88 & 0.8085 & 0.0327 & 0.0971 & 0.0025 & 0.0604 & 0.0024 & 602 & 18 & 597 & 15 & 618 & 86 & 599 & 13 \\
\hline 89 & 4.8100 & 0.2406 & 0.3149 & 0.0105 & 0.1108 & 0.0029 & 1787 & 42 & 1765 & 52 & 1812 & 48 & 1779 & 38 \\
\hline 90 & 10.5631 & 0.3155 & 0.4558 & 0.0082 & 0.1681 & 0.0034 & 2485 & 28 & 2421 & 36 & 2539 & 34 & 2464 & 25 \\
\hline 93 & 0.7076 & 0.0321 & 0.0905 & 0.0019 & 0.0567 & 0.0017 & 543 & 19 & 559 & 11 & 479 & 65 & 556 & 11 \\
\hline 94 & 0.7479 & 0.0233 & 0.0859 & 0.0018 & 0.0631 & 0.0019 & 567 & 14 & 531 & 11 & 712 & 63 & 543 & 10 \\
\hline 95 & 0.7540 & 0.0245 & 0.0952 & 0.0015 & 0.0574 & 0.0019 & 571 & 14 & 586 & 9 & 509 & 71 & 583 & 8 \\
\hline
\end{tabular}

(a)

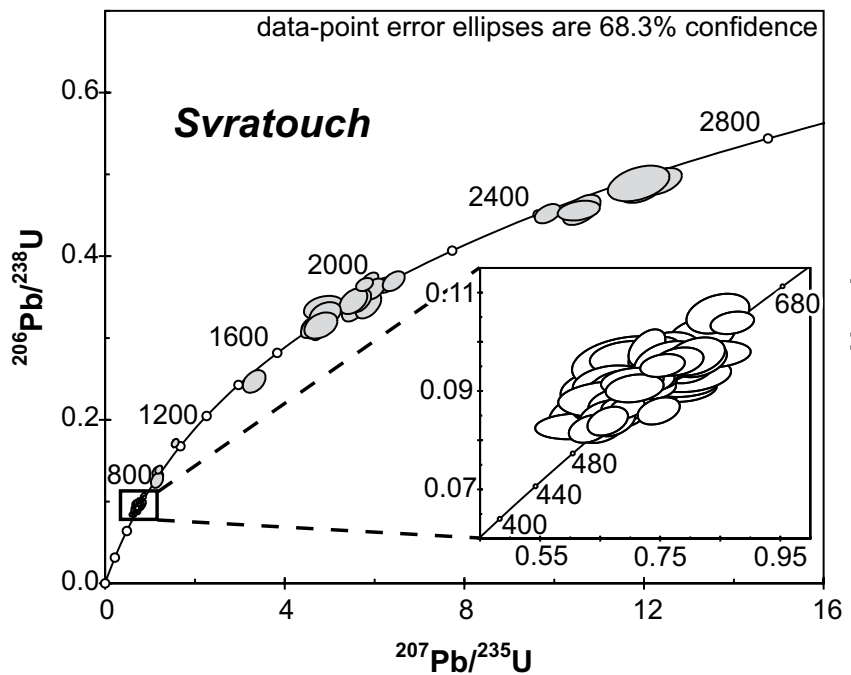

(b)

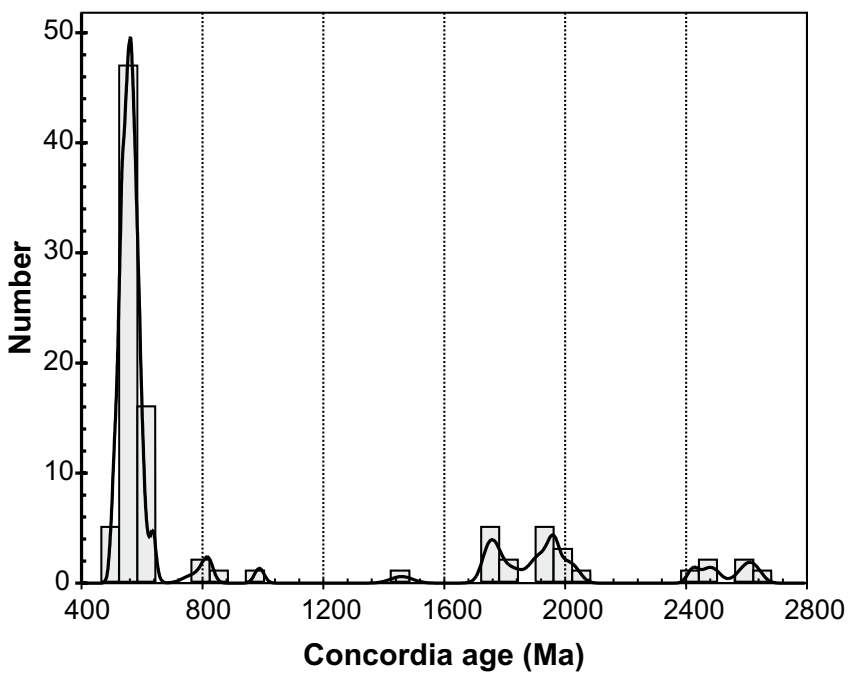

Fig. 12a - Concordia diagram for concordant population of detrital zircons from the Svratouch sample. Lower-right inset presents the measured data in zoomed portion of the Concordia diagram for interval between 400 and $700 \mathrm{Ma}$; $\mathbf{b}$ - Frequency curve and histogram of zircon ages for the same sample as in (a). 
than in titanites described from China. Nevertheless, the decompression-related symplectites in our samples, composed of titanite II and plagioclase, clearly formed from early titanite I with increased $\mathrm{Al}$, and were preserved locally as small relics.

The proposed P-T-t evolutionary scheme for the skarn bodies and surrounding rocks is based on integrated data on petrology, geochronology and the structural patterns. On a regional scale, there are preserved some unequivocal relics of the original intrusive contacts between the Svratka metagranites and the surrounding migmatites (Verner et al. this volume). An old generation of isoclinal and rootless folds in the skarns and migmatites pre-dates this intrusion (Verner et al. this volume). As a consequence of widespread and intense Variscan deformation and recrystallization of paragneisses and migmatites in the Svratka Unit, relict domains of preVariscan mineral assemblages are rare. The emplacement of Svratka metagranite was dated at $\sim 515 \mathrm{Ma}$ (Schulmann et al. 2005). These rocks were affected only by a relatively weak deformation and partial recrystallization during the Variscan Orogeny (see Buriánek et al. this volume).

\subsubsection{Moldanubian Zone}

The pressure-temperature conditions in the northern part of the Moldanubian Zone were estimated for rocks in the proximity of skarns, i.e., calc-silicate gneisses, granulites and migmatized paragneisses. Pertoldová et al. (2007) reported P-T conditions for three stages of metamorphism. Phase $\mathrm{M}_{1}$ corresponded to the peak metamorphic conditions $\left(\mathrm{T}=660^{\circ} \mathrm{C}\right.$ and $\left.\mathrm{P}=0.6 \mathrm{GPa}\right)$. The $\mathrm{P}-\mathrm{T}$ conditions for the HT/LP phase $M_{2}$, leaving its imprint in impure marbles, corresponded to $550{ }^{\circ} \mathrm{C}$ and $0.2 \mathrm{GPa}$ (Novák 1989). The retrograde metamorphism $M_{3}$ resulted in crystallization of chlorite, replacing amphibole, plagioclase and epidote. The presence of the younger andradite-rich garnet generation, cutting the older, corroded garnet grains, indicates a sharp increase in oxygen fugacity, most probably caused by ingress of hydrothermal fluids. It is so far uncertain whether the hydrothermal fluids were igneous-related or of regional metamorphic origin.

Tajčmanová et al. (2006) assessed the P-T conditions of equilibration in granulites and migmatized paragneisses. The P-T conditions were estimated at $\sim 850^{\circ} \mathrm{C}, 1.8 \mathrm{GPa}$ for the granulites, and at $870^{\circ} \mathrm{C}$ and $0.8-1.1 \mathrm{GPa}$ for migmatized paragneisses. These authors described the polyphase retrogressive character of decompression, corresponding to a temperature of $620^{\circ} \mathrm{C}$ and pressure of 0.4-0.6 GPa. Schulmann et al. (2008) also reported results for several samples documenting prograde and retrograde paths of metamorphism (see Fig. 13).

\subsubsection{Kutná Hora Complex}

Vrána et al. (this volume) deciphered three metamorphic events in gneisses, migmatites and granulites of the Běstvina Unit (Kutná Hora Complex). Event $M_{1}$ resulted in crystallization of $\mathrm{HP} / \mathrm{HT}$ granulites in the Běstvina Unit at $840-920^{\circ} \mathrm{C}$ and $1.8-2.2 \mathrm{GPa}$ (i.e., eclogite-facies conditions). Nahodilová et al. (2006) reported peak conditions of $831 \pm 53{ }^{\circ} \mathrm{C}$ and $1.65 \pm 1.8 \mathrm{GPa}$ followed by retrogression at $705 \pm 97^{\circ} \mathrm{C}$ and $1.4 \pm 0.2 \mathrm{GPa}$, based on study of different samples from the Běstvina Unit. For the second event $\mathrm{M}_{2}$, Nahodilová et al. (2006) estimated the P-T conditions in migmatites at $875 \pm 95^{\circ} \mathrm{C}$ and 1.56 $\pm 0.14 \mathrm{GPa}$. Data for MP/LT retrogression indicate $\mathrm{T}=$ $712 \pm 39{ }^{\circ} \mathrm{C}$ and $\mathrm{P}=1.06 \pm 0.18 \mathrm{GPa}$. The P-T path of eclogite from the new Roztěz locality in the KHC was estimated by means of the pseudosection method. The modelling indicated minimum pressures above 2.15-2.30 $\mathrm{GPa}$ and temperatures $600-650{ }^{\circ} \mathrm{C}$ reached during the prograde part of metamorphism (Štědrá and Nahodilová this volume). The high-jadeite clinopyroxene in the kyanite-bearing eclogite from Bořetice indicates even higher pressures than those inferred from the Roztěž eclogite and from the Běstvina Unit $-761^{\circ} \mathrm{C} / 4.3 \mathrm{GPa}$ (Štědrá and Nahodilová this volume)

Metamorphic event $\mathrm{M}_{3}$ was characterized by pressure of 0.9 to $1.2 \mathrm{GPa}$ in the Ky-Grt felsic gneisses (Vrána et al. this volume). No suitable mineral pair was found for a temperature estimate.

The multistage tectonometamorphic history of the Kutná Hora Complex has already been assumed by Synek and Oliveriová (1993) but their study lacked exact geothermobarometric data. Observations by these authors as well as by Nahodilová et al. (2006) and Vrána et al. (this volume) show that the metamorphic development of the Kutná Hora Complex was essentially different from that of the Moldanubian Zone. In the former unit, decompression took place mainly in the kyanite stability field. On the other hand, decompression in the Moldanubian Zone was marked by crystallization of sillimanite, replacement of kyanite by spinel or low-Ca garnet and production of cordierite (Tajčmanová et al. 2006).

\subsection{Geochemistry}

The studied skarn samples from the Svratka Unit, the Moldanubian Zone and the Kutná Hora Complex experienced a complicated history of polyphase metamorphism. Geochemical data document large variability in the major- and trace-element compositions, including REE, among individual localities and also among samples from the same locality. The differences in REE abundances cannot be explained solely on the basis of differences in clinopyroxene and garnet contents, or 


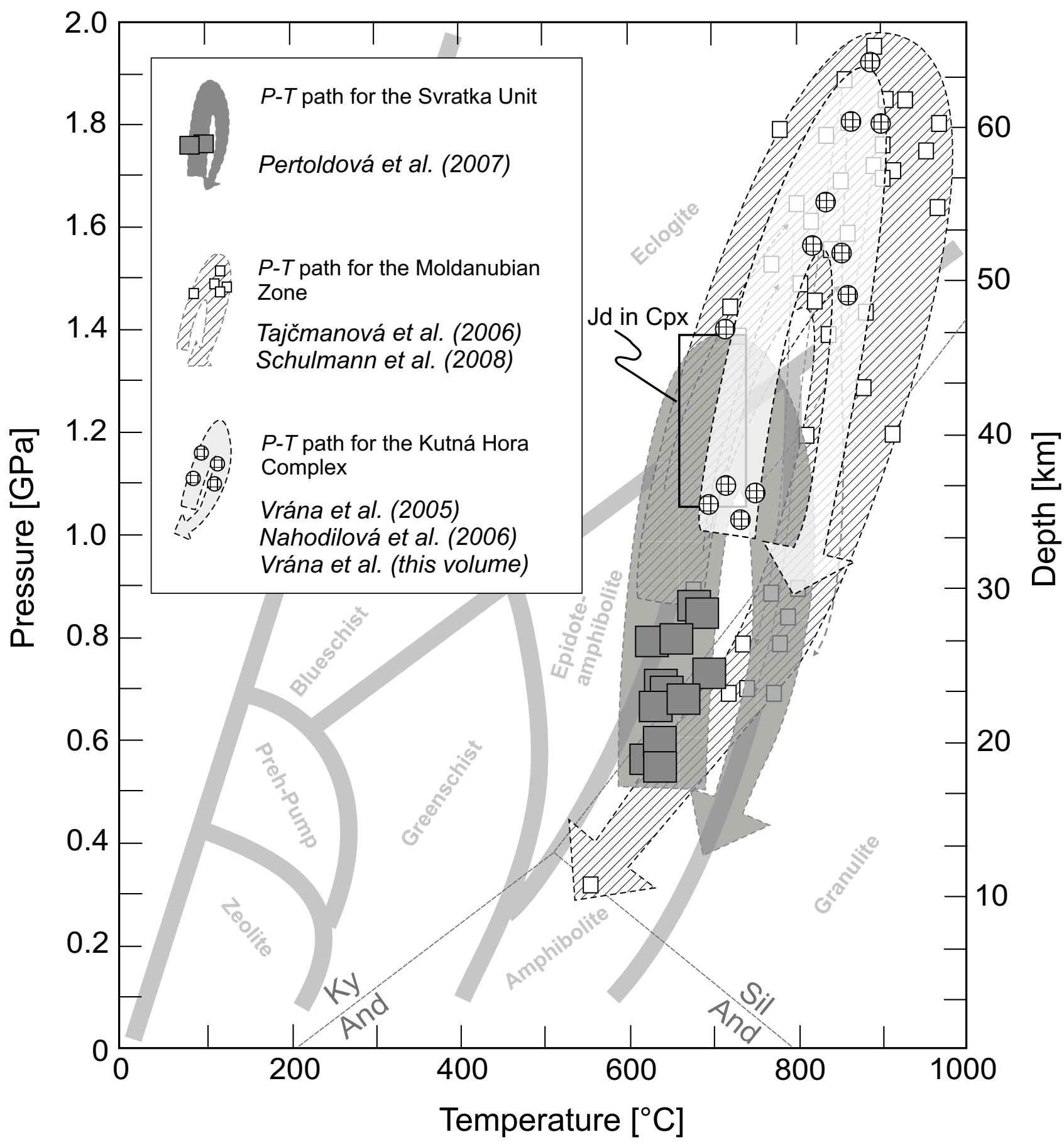

Fig. 13 Summary of the published P-T paths for the Svratka Unit, the Moldanubian Zone and the Kutná Hora Complex.

indeed any other rock-forming minerals, as the mobility of the most elements was strongly limited during Variscan metamorphism. Arguments pointing to the conservative character of skarn metamorphism (closed system) have already been published by Pertold et al. (1997). They include, for instance, different garnet and pyroxene chemistry, distinct whole-rock LREE/HREE ratios, and oxygen isotope compositions preserved in individual skarn bands. Consequently, we consider the geochemical variability of individual skarn types (bands, layers) to be a close approximation to the original compositional/lithological variations of the pre-metamorphic protoliths.

Skarns from all three studied units share some common geochemical features. The $\mathrm{Al}_{2} \mathrm{O}_{3} / \mathrm{TiO}_{2}, \mathrm{Al}_{2} \mathrm{O}_{3} / \mathrm{Zr}, \mathrm{TiO}_{2} / \mathrm{Nb}$ ratios are closely similar to the average composition of 
post-Archaean sediments but the elemental abundances are usually lower in the skarns. This suggests that skarn protoliths contained, apart from variable proportions of detrital sedimentary material, also other, non-detrital components. These admixtures were mainly $\mathrm{Ca}$ and $\mathrm{Fe}$, supplied by chemogenic sedimentation. However, the balance of the latter elements is rather variable in our skarn set. The absence of correlation between $\mathrm{FeO}_{t}$ and $\mathrm{CaO}$ indicates that the two elements do not substitute for each other and that there was no replacement of $\mathrm{Ca}$ by $\mathrm{Fe}$ in the skarn protoliths.

The $\Sigma$ REE values in the skarns ( 8 to $345 \mathrm{ppm}$ ) are both higher and lower than total REE contents in average sediments $(121 \mathrm{ppm})$. If the abundances of $\mathrm{Al}_{2} \mathrm{O}_{3}, \mathrm{TiO}_{2}$ and $\mathrm{Zr}$ are considered as indicators for detrital sedimentary components in skarn protoliths, it is obvious from diagrams of $\mathrm{Al}_{2} \mathrm{O}_{3}$ vs. $\Sigma \mathrm{REE}$ and $\mathrm{TiO}_{2}$ vs. $\Sigma \mathrm{REE}$ (Fig. 9) that the skarns contain mostly lower values of $\mathrm{Al}_{2} \mathrm{O}_{3}$ and $\mathrm{TiO}_{2}$ compared to average sediments. However, their ratios to $\Sigma$ REE point to relative enrichment of REE in the detritic component in the skarns. Comparison with paragneisses and migmatites of the Strážek Unit (Moldanubian Zone) (Hrdličková ed. 2008) and paragneisses of the Svratka Unit (Hanžl ed. 2008) shows that the latter have higher $\mathrm{Al}_{2} \mathrm{O}_{3} / \mathrm{TiO}_{2}$ and $\mathrm{Al}_{2} \mathrm{O}_{3} / \mathrm{Zr}$ ratios than Moldanubian paragneisses, skarns and average sediments. It is probable that formation of skarn protoliths included some processes resulting in relative enrichment in REE, which took place in a sedimentation environment with a lower supply of detrital material. This would account for the situation that many skarn samples contain $\Sigma \mathrm{REE}$ higher, but $\mathrm{Al}_{2} \mathrm{O}_{3}$, $\mathrm{TiO}_{2}$ and $\mathrm{Zr}$ lower, than the average sediments.

The lowest total REE contents (less than $100 \mathrm{ppm}$ ) occur in skarns that are known as former magnetite ore deposits or at least contain some magnetite accumulations (Zliv, Županovice, Rešice, Budeč, Vepřová, Kuklík, Pernštejn, Svratouch and Malešov). Skarns at Církvice and Jakub near Církvice also have such low $\Sigma$ REE values, although no magnetite is known at present from these localities.

Europium anomalies in skarn samples have a wide range of 0.5-8.6. In many cases there are differences among samples from the same locality. The Fig. 10 indicates that the most pronounced positive anomalies of Eu occur predominantly in samples with the lowest $\Sigma$ REE values. At the same time, these are the samples with the lowest contents of $\mathrm{Al}_{2} \mathrm{O}_{3}$ (less than $6 \%$ wt. \%), $\mathrm{TiO}_{2}$ (less than 0.25 wt. \%) and $\mathrm{Zr}$ (less than $100 \mathrm{ppm}$, with a few exceptions) and, in our interpretation, the samples with the lowest detrital component. The occurrence of a positive Eu anomaly in sediments and hydrothermal products is usually interpreted as resulting from the activity of reducing, high-temperature fluids. The presence of positive $\mathrm{Eu}$ anomalies in some skarn samples is in contrast with the negative europium anomaly in metasediments of the Moldanubian Zone and of the Svratka Unit.

Hongo et al. (2007) recorded a distinct U-shaped REE patterns with a prominent positive Eu anomaly in hydrothermal fluids from the sea-floor black smoker (Fig. 8). These geochemical characteristics are in sharp contrast to the sea water, which does not have a Eu anomaly and the REE are not fractionated, except for Ce. According to Zheng et al. (2008), marine exhalites may exhibit enrichment in lanthanide elements by two or three orders of magnitude relative to the sea water, due to the hydrothermal solutions activity and adsorption phenomena. Oxidation processes take place in dependence on the distance from the source of solutions and residence time on the sea floor.

Some of the analysed skarns contain increased abundances of base metals: $\mathrm{Cu}$ (Budeč), $\mathrm{Zn}, \mathrm{Pb}$ (Budeč, Zliv, Županovice, Býšovec, Kadov, Kuklík, Malešov), As (Budeč, Svratouch, Pernštejn, Kuklík), Bi and Au (Budeč, Zliv, Županovice, Církvice, Ruda near Čachnov, Svratouch, Kuklík). The tin contents are somewhat increased in all the skarn samples. The highest values were recorded in Budeč and Slatina (400 to $570 \mathrm{ppm}$ ), in Církvice, Jakub near Církvice, Líšné, Točice and Rešice (200 to 360 ppm), as well as in Vepřové, Pernštejn, Zliv, Čachnov, Teplá and Malešov (100 to 200 ppm). Skarns from other localities contain $\mathrm{Sn}$ in abundances that are one order of magnitude higher than in the metasediments of the Svratka Unit and the Moldanubian Zone (Hanžl ed. 2008; Hrdličková ed. 2008).

\subsection{Oxygen isotopes}

The $\delta^{18} \mathrm{O}$ values for garnet and clinopyroxene are low, on average $1.62 \%$ for garnet, $2.2 \%$ for clinopyroxene and $2.15 \%$ for amphibole. Such compositions cannot be interpreted as resulting from crystallization of the mineral assemblages in the presence of granitoidrelated magmatic fluids, i.e. during contact metamorphism. It is probable that a component of marine or meteoritic water was involved. The new data compare well with the results published by Pertoldová et al. (1998), Pertold et al. (1997), and Drahota et al. (2005) for other skarn localities in the Moldanubian Zone and the Svratka Unit. The determined $\delta^{18} \mathrm{O}$ values for minerals from the Vrbík small contact metasomatic scheelite deposit (Pertold et al. 2000) and from the Vápenka contact skarn band (Drahota et al. 2005) exhibit distinctly higher heavy oxygen contents. The $\delta^{18} \mathrm{O}$ values published by Meinert et al. 2003 for skarn deposits connected with igneous-related hydrothermal fluids are higher as well (Meinert et al. 2003: average $\delta^{18} \mathrm{O}$ of $5.0 \%$ for garnet, $6.5 \%$ for clinopyroxene, and $7.1 \%$ for amphibole). 


\subsection{Geochronological data}

The ages obtained from the detrital zircon population suggest that the youngest source of clastic material for the studied sample was of Cambrian-Neoproterozoic age. The most pronounced age maximum spans the interval of 500-640 Ma with maximum data density between 540-600 Ma. The Neoproterozoic protolith ages of 540-580 Ma are typical of granitoids and orthogneisses of the Brunovistulian (van Breemen et al. 1982; Scharbert and Batík 1985; Fritz et al. 1996; Friedl et al. 2000, 2004; see Leichmann and Höck 2008 for review) as well as Saxothuringian and Lugian domains (Hegner and Kröner 2000; Tichomirowa et al. 2001; Linnemann et al. 2008 and references therein) and they only rarely appear as protolith ages of Moldanubian orthogneisses (Schulmann et al. 2005). Importantly, Cambrian ages of 500-520 Ma are absent in the Moravo-Silesian Domain (Brunia), but they are known as the most frequent protolith ages of the Moldanubian orthogneisses, as well as of some orthogneisses in the Saxothuringian and Lugian domains (Vrána and Kröner 1995; Hegner and Kröner 2000; Tichomirowa et al. 2001; Friedl et al. 2004; Schulmann et al. 2005). Zircons of this age found in the studied samples can be interpreted either as clastic grains coming directly from eroded Cambrian granitoids or volcanics, or they represent recycled crystals from sediments rich in clastic material of Cambrian age. Ages older than $650 \mathrm{Ma}$ are known from xenocrystic cores of younger zircons in Brunovistulian, Saxothuringian and Lugian orthogneisses as well as from orthogneisses and granulites of the Moldanubian Domain (Hegner and Kröner 2000; Kröner et al. 2000; Tichomirowa et al. 2001; Friedl et al. 2004 and references therein).

The presence of Cambrian zircons with an age of $\sim 500$ Ma shows that the deposition of the sedimentary protolith to the skarn sample occurred during Early Palaeozoic but not earlier than in the Mid-Late Cambrian times. Tectonometamorphic activity associated with the Variscan collision in the Bohemian Massif is known to be Mid-Late Devonian (Franke 2000) and this period can probably be regarded as the minimum age for the protolith sedimentation. The minimal age of metamorphism in the studied sample is constrained by the cooling ages from the associated gneisses, the Ar-Ar muscovite and amphibole age of which is Early Carboniferous (330-326 Ma; Fritz et al. 1996).

Interpretation of the sediment provenance is complicated by the fact that ages recognized in the detrital population are known from many geological units of the Bohemian Massif except for the Moldanubian Domain. The opening of a large oceanic domain (the Saxothuringian ocean) started during the Early-Middle Cambrian, resulting in separation of the Saxothuringian/
Lugian domains from the more easterly exposed units of the Bohemian Massif (Franke 2000). Given the major shortening of the Moldanubian Domain during the Variscan continental collision in the Late Devonian-Early Carboniferous, it seems unlikely that the Saxothuringian/Lugian domains acted as sediment source regions. They seem to have been remote from the Moldanubian Domain at the time of assumed sedimentation of the protolith, e.g. between Late Cambrian and Late Devonian. So far there is no reliable evidence for separation of the Brunovistulian and Moldanubian domains by a large ocean. The palaeogeographic reconstruction, together with the dense population of zircons with ages between 540 and $580 \mathrm{Ma}$, support the interpretation that much of the clastic material could have been derived from sources in the neighbouring Brunovistulian Domain. ArchaeanMesoproterozoic ages probably correspond to recycled zircons, either xenocrystic cores of magmatic zircons in Neoproterozoic granitoids, or clastic zircons in the sediments of Neoproterozoic-Cambrian age.

\section{Conclusions}

- Skarns form elongated lenticular bodies that are subparallel to regional fabrics in the surrounding rocks. The skarns have preserved the relict fabrics, which are in some cases overprinted by regional structures.

- Variations in mineral chemistry correspond to differences in the chemical compositions of the protoliths.

- The temperature or P-T conditions of the skarn mineral assemblages were determined in all three units. These conditions are compared to P-T intervals of metamorphic paths recognized in surrounding rocks.

- Three metamorphic stages $\left(M_{1}\right.$ - early prograde, $M_{2}$ - prograde/peak, $\mathrm{M}_{3}$ - retrograde) were determined in the skarns from the Svratka Unit, with minimal peak pressures of $1.4 \mathrm{GPa}$.

- The P-T conditions in skarns from the Moldanubian Zone are represented by a narrow interval within the amphibolite facies. The peak pressure conditions of metamorphism were not preserved and compositional zoning in garnets shows signs of retrograde Variscan evolution.

- Chemical zoning of some garnets from the Kutná Hora Complex indicates prograde development under amphibolite-facies conditions but peak pressure conditions were not preserved, similarly to the Moldanubian Zone. The chemically homogeneous compositions of individual generations of clinopyroxenes and epidotes do not reflect the prograde and retrograde events in the Kutná Hora Complex.

- In all three units, a local increase in the oxygen fugacity in the system played a significant role in 
development of minerals rich in $\mathrm{Fe}^{3+}$, especially magnetite.

- The estimated P-T conditions for the skarns are in compliance with the metamorphic paths of the surrounding metasediments in all the studied units, except the peak pressures in the Svratka Unit. These results indicate that the protolith of skarns endured all the metamorphic events together with the surrounding sedimentary/volcano-sedimentary sequences.

- The light oxygen isotopic compositions in skarn silicates from the Svratka Unit do not support the idea of an open-system genesis due to a granitoid-magma derived fluid activity. The interaction with seawater was probable during the formation of the skarn protoliths.

- The large variability of the zircon ages $(0.5-2.6 \mathrm{Ga})$ suggests their detrital origin. The most frequent ages fall in the interval of 500-640 Ma with majority of data clustering between 540-600 Ma. Zircons and other clastic material are therefore Early Palaeozoic $(>500 \mathrm{Ma})$ in age and possibly linked to a source in rocks of Cambrian-Neoproterozoic age.

- Skarn protoliths were most probably mixed sedimentary rocks with a component of exhalites deposited on the sea floor. In addition to enrichment in $\mathrm{Ca}$ and $\mathrm{Fe}$, and lowered $\mathrm{Si}$, they contain increased abundances of some metals ( $\mathrm{Zn}, \mathrm{Pb}, \mathrm{As}, \mathrm{Sn}$ ). The total REE contents with the type and magnitude of the Eu anomaly are highly variable. They indicate variation in the local temperature and redox conditions among individual layers at a single locality as well as regional changes between individual localities. There is also an indication of rather conservative character of the metamorphism with limited migration of most geochemical species within the garnet-clinopyroxene rocks. It is probable that hydrothermal solutions, instrumental for the development of skarn protoliths, debouched on the sea floor along fracture zones with the character of local extensional rifts. The deposition of exhalites proceeded along such zones, where they were mixed with detrital material in variable proportions.

- The geochemical features of the studied skarns do not indicate systematic differences among the three studied geological units, although the tectono-metamorphic development was indeed different. The sedimentaryexhalative protoliths of the skarns were mineralized by magnetite at some localities, giving rise to small deposits of iron ore, accompanied by a slight enrichment in some base metals, as is common in this type of mineralization.

- The geological positions of the skarn bodies and their structural and metamorphic record reflect probable collateral tectono-metamorphic development of skarns with the surroundings rocks. The geochemical signatures, the oxygen isotopic compositions and the presence of detrital zircons with a wide range of ages exclude metasomatic, and instead point to a sedimentary-exhalative, mode of origin for the studied skarns.

Acknowledgements This research was supported by Project No. 6352 of the Ministry of Environment and Internal Research Projects No. 3259 and No. 3270 of the Czech Geological Survey. We are indebted to Stanislav Vrána for his helpful discussions and constructive comments.

Madeleine Štulíková is thanked for revising the English of this manuscript, František Veselovský for separation of zircons and Renata Čopjaková, Radek Škoda with Petr Sulovský for assistance with the electron microprobe analysis. We appreciate the help of the Journal of Geosciences editor-in-chief Vojta Janoušek and reviewers, Larry Meinert and anonymous, to improve the manuscript. Last but not least, we thank W. S. Faryad for the careful editorial handling.

Electronic supplementary material. The GPS coordinates of the studied samples, and the tables of whole-rock geochemical data (Tabs 9-11) are available online at the Journal web site (http://dx.doi.org/10.3190/jgeosci.044).

\section{References}

Boynton WV (1984) Geochemistry of the rare-earth elements: meteorite studies. In: HENDERson P (ed) Rare Earth Element Geochemistry. Elsevier, Amsterdam, pp 63-114

BuRIÁNEK D (2008) Mafic enclaves from the diatexite migmatites in the Svratka Crystalline Complex. Acta Mus Moraviae, Sci Geol 93: 137-150

Burí́nek D, Čopjaková R (2008) Tourmaline from the mica schist of the Svratka Crystalline Complex. Acta Mus Moraviae, Sci Geol 93: 61-79

Buriánek D, Verner K, Hanžl P, Krumlová H (2009) Ordovician metagranites and migmatites of the Svratka and Orlice-Sněžník units, northeastern Bohemian Massif. J Geosci 54: 181-200

BURT DM (1982) Skarn deposits - historical bibliography through 1970. Econ Geol 77: 755-763

CARSWELl DA, WiLSON RN, ZHAi M (1996) Ultra-high pressure aluminous titanites in carbonate-bearing eclogites at Shuanghe in Dabieshan, Central China. Mineral Mag 60: 461-471

CAWTHORn RG, ColLerson KD (1974) The recalculation of pyroxene end-member parameters and the estimation of ferrous and ferric iron content from electron microprobe analyses. Amer Miner 59: 1203-1208

Cháb J, Stráník Z, Eliáš M, Adamovič J, Babůrek J, Breiter K, Cajz V, Domečka K, Fišera M, Hanžl P, Holub V, Hradecký P, Chlupáč I, Klomínský J, Mašek 
J, Mlčoch B, Opletal M, Otava J, Pálenský P, Prouza V, RƯŽIČKa M, SchovÁNeK P, SlabÝ J, VALeČKa J, ŽÁČEK V (2007) Geological map of Czech Republic 1:500 000 (uncovered). Czech Geological Survey, Prague, ISBN 978-80-7075-699-7

Chavagnac V, German C, Milton A, Palmer MR (2005) Sources of REE in sediment cores from the Rainbow vent site (36 $\left.{ }^{\circ} 14^{\prime} \mathrm{N}, \mathrm{MAR}\right)$. Chem Geol 216: 329-352

Clayton RN, Mayeda TK (1963) The use of bromine pentafluoride on the extraction of oxygen from oxides and silicates for isotopic analysis. Geochim Cosmochim Acta 27: 43-52

Coggon R, Holland TJB (2002) Mixing properties of phengitic micas and revised garnet-phengite thermobarometers. J Metamorph Geol 20: 683-696

CondiE KC (1993) Chemical composition and evolution of the upper continental crust: contrasting results form surface samples and shales. Chem Geol 104: 1-37

Deer WA, Howie RA, Zussman J (1966) An Introduction to Rock Forming Minerals. Longman, London, pp 1-528

Deer WA, Howie RA, Zussman J (1972) Rock Forming Minerals. Vol. 1, Ortho- and Ring Silicates. Longman, London, pp 77-112

Drahota P, Pertold Z, Pudilová M (2005) Three types of skarn in the northern part of the Moldanubian zone, Bohemian Massif - implications for their origin. J Czech Geol Soc 50: 19-33

DROOP GTR (1987) A general equation for estimating $\mathrm{Fe}^{3+}$ concentrations in ferromagnesian silicates and oxides from microprobe analyses, using stoichiometric criteria. Mineral Mag 51: 431-435

Edel JB, Schulmann K, Holub FV (2003) Anticlockwise and clockwise rotations of the Eastern Variscides accommodated by dextral lithospheric wrenching: palaeomagnetic and structural evidence. J Geol Soc, London 160: 209-218

Einaudi MT, Meinert LD, Newberry RJ (1981) Skarn deposits. Econ Geol 77: 317-391

Franke W (1989) Variscan plate tectonics in Central Europe - current ideas and open questions. Tectonophysics 169: $221-228$

Franke W (2000) The mid-European segment of the Variscides: tectonostratigraphic units, terrane boundaries and plate tectonic evolution. In: Franke W, HAAK V, Oncken O, TANner D (eds) Orogenic Processes: Quantification and Modelling in the Variscan Belt. Geol Soc London Spec Publ 179: 63-86

Friedl G, Finger F, McNaughton NJ, Fletcher IR (2000) Deducing the ancestry of terranes: SHRIMP evidence for South America-derived Gondwana fragments in Central Europe. Geology 28: 1035-1038

Friedl G, Finger F, Paquette JL, von Quadt A, McNaughtON NJ, Fletcher IR (2004) Pre-Variscan geological events in the Austrian part of the Bohemian Massif deduced from U-Pb zircon ages. Int J Earth Sci 93: 802-823

Fritz H, Dallmeyer RD, Neubauer F (1996) Thick-skinned versus thin-skinned thrusting: rheology controlled thrust propagation in the Variscan collisional belt (the southeastern Bohemian Massif, Czech Republic-Austria). Tectonics 15: 1389-1413

Gaspar M, Knaack C, Meinert LD, Moretti R (2008) REE in skarn systems: a LA-ICP-MS study of garnets from the Crown Jewel gold deposit: Geochim Cosmochim Acta 72: $185-205$

GaspariK T, LindSLEy DH (1980) Phase equilibria at high pressure of pyroxenes containing monovalent and trivalent ions. In: Prewit CT (ed) Pyroxenes. Mineral Soc Am Rev Mineral 7: 309-339

Hanžl P, Buriánek D, Čurda J, Fürich V, HrdičKová K, Kirchner K, Lysenko V, Mrnková J, Otava J, PerTOLdOVÁ J, RoštínskÝ P, RÝdA K, SKÁcelová Z, Vít J, ZELENKA P (2008) Basic geological map ČR 1:25000 with explanations, 14-333 Svratka. Czech Geological Survey, Prague, pp 1-72 (in Czech)

Hanžl P, HrdličKová K, Bešta J, BlažKová Š, BŘízová E, Dosbaba M, Fürych V, Kirchner K, Kryštofová E, LySEnKo V, Pertoldová J, RoštínskÝ P, RÝda K, Skácelová Z, Skácelová D, Tajčmanová L, Vít J, ZavŘelová A, ŽÁČKovÁ E (2009) Basic geological map ČR 1:25000 with explanations, 24-113 Nové Město na Moravě. Czech Geological Survey, Prague, pp 1-62 (in Czech)

Hegner E, KRÖNER A (2000) Review of Nd isotopic data and xenocrystic and detrital zircon ages from the pre-Variscan basement in the eastern Bohemian Massif: speculations on palinspastic reconstructions. In: Franke W, HAAK V, OnCKen O, Tanner D (eds) Orogenic Processes: Quantification and Modelling in the Variscan Belt. Geol Soc London Spec Publ 179: 113-129

Hoefs J (2009) Stable Isotope Geochemistry. SpringerVerlag, Berlin, pp 1-285

Holland TJB, Powel R (1998) An internally consistent thermodynamic data set for phases of petrological interest. J Metamorph Geol 16: 309-343

Holland T, Powell R (2003) Activity-composition relations for phases in petrological calculations: an asymmetric multicomponent formulation. Contrib Mineral Petrol 145: 492-501

Hongo Y, Obata H, Gamo T, Nakasema M, Ishibashi J, Konno U, Saegusa S, Ohrubo S, Tsunogai U (2007) Rare earth elements in the hydrothermal system at Okinawa Trough back-arc basin. Geochem J 41: 1-15

HouZAR S, ŠReIN V (1995) Manganese-bearing skarn from Meziřričko near Želetava. Sbor přírodověd klubu Západomor muzea 20: 1-9

HrdličKová K, BŘizová E, Fürych V, Hanžl P, Kadlecová R, Kirchner K, Lysenko V, Lhotský P, Mrnková J, Pertoldová J, Roštínský P, Skácelová D, Skácelová 
Z, VíT J (2008) Basic geological map ČR $1: 25000$ with explanations, 23-224 Žd'ár nad Sázavou. Czech Geological Survey, Prague, pp 1-60 (in Czech)

Jamtveit B, Wogelius RA, Fraser DG (1993) Zonation patterns of skarn garnets, records of hydrothermal system evolution. Geology 21: 113-116

JANOUŠEK V, FARROW CM, ERBAN V (2006) Interpretation of whole-rock geochemical data in igneous geochemistry: introducing Geochemical Data Toolkit (GCDkit). J Petrol 47: 1255-1259

KlomínsKÝ J, SatTRAn V (1963) Entstehungsgeschichte der Skarne im mittleren Teil von Krušné Hory (Erzgebirge). Věst ústř Úst geol 38: 341-374

Košler J, Fonneland H, Sylvester P, Tubrett M, Pedersen RB (2002) U-Pb dating of detrital zircons for sediment provenance studies - a comparison of laser ablation ICPMS and SIMS techniques. Chem Geol 182: 605-618

KošLeR J, Sylvester P. J (2003) Present trends and the future of zircon in geochronology: laser ablation ICP-MS. In: Hanchar JM, Hoskin PWO (eds) Zircon. Mineralogical Society of America Reviews in Mineralogy and Geochemistry 53: 243-275

KоткоVÁ J (1991) Skarns of the central part of the Krušné Hory Mts. - mineralogy, geochemistry and their implications for the skarn origin. Bull Czech Geol Surv 66: 215-232

KouteK J (1950) Deposit of skarn-type at Vlastějovice in the area around the Sázava River. Rozpr Čs Akad Věd, ř mat př́r Věd 60: 1-30 (in Czech)

Kröner A, O’Brien PJ, Nemchin AA, Pidgeon RT (2000) Zircon ages for high pressure granulites from South Bohemia, Czech Republic, and their connection to Carboniferous high temperature processes. Contrib Mineral Petrol 138: 127-142

Leichmann J, Höck V (2008) The Brno Batholith: an insight into the magmatic and metamorphic evolution of the Cadomian Brunovistulian Unit, eastern margin of the Bohemian Massif. J Geosci 53: 281-305

Linnemann U, Pereira F, Jeffries Te, Drost K, Gerdes A (2008) The Cadomian orogeny and the opening of the Rheic Ocean: the diacrony of geotectonic processes constrained by LA-ICP-MS U-Pb zircon dating (OssaMorena and Saxo-Thuringian Zones, Iberian and Bohemian massifs). Tectonophysics 461: 21-43

Litochleb J, Novák J, Šrein V (1997) Chemical composition of silicates from garnet-magnetite skarn from Horní Ves near Mariánské Lázně (Marienbad). Bull mineral-petrolog odd Nár muz (Praha) 4-5: 153-154 (in Czech)

LuDWIG KR (1999) IsoplotEx v 2.6. Berkeley Geochronological Center Special Publication, Los Angeles, 1a: pp 1-47

MeINERT LD (1998) A review of skarns that contain gold. In: LeNTz DR (ed) Mineralized Porphyry/Skarn Systems. Min Assoc Can Short Course Series 26: 359-414
Meinert LD, Hedenquist JW, Satoh H and Matsuhisa Y (2003) Formation of anhydrous and hydrous skarn in $\mathrm{Cu}-\mathrm{Au}$ ore deposits by magmatic fluids. Econ Geol 98: $147-156$

Meinert LD, Dipple GM and Nicolescu S (2005) World Skarn Deposits. In: Hedenquist JW, Thompson JFH, Goldfarb RJ, Richards JP (eds) Economic Geology $100^{\text {th }}$ Anniversary Volume, Society of Economic Geologists, Littleton, Colorado, USA, Includes supplementary appendices on CD-ROM (filename: Meinert), pp 299-336

Melichar R, Břizová E, Buriánek D, Čurda J, Fürych V, Hanžl P, HrdličKová K, KirChNer K, Lysenko V, MrNKOVÁ J, RošTínSKÝ P, RÝdA K, SKÁCELOVÁ Z, Vít J (2008) Basic geological map ČR 1:25000 with explanations, 24-111 Sněžné. Czech Geological Survey, Prague, pp 1-58 (in Czech)

Nahodilová R, Faryad S, Pertoldová J, Konopásek J, ŠTĚDRÁ V (2005) HP melting and its relationship to the granulite facies metamorphism - an example of the „Gföhl nappe“ in the Kutná Hora Crystalline Complex. Geolines 19: 86-87

Nahodilová R, Faryad S, Pertoldová J, Konopásek J, ŠTĚDRÁ V (2006) Contrasting metamorphic evolution of HP rocks in the Gföhl Unit of the Kutná Hora Crystalline Complex and in the Moldanubian Zone in Austria. Geolines 20: 98-99

NĚMEC D (1991) Regional typization of the iron skarns of the Bohemian-Moravian Heights (Českomoravská vrchovina). Acta Mus Morav, Sci Nat 76: 51-82

Nicolescu S, Cornell DH, Sodervall U and Odelius H (1998) Secondary ion mass spectrometry analysis of rare earth elements in grandite garnet and other skarn related silicates. Eu J Mineral 10: 251-259

NovÁk M (1989) Metamorphism of dolomitic rocks at the North-East margin of the Moldanubicum. Acta Mus Morav Sci Nat 74: 7-51

Pertold Z, Pouba Z (1982) Prävariszische Mineralisationen der peripheren Zone des Böhmischen Massivs (ČSSR). $Z$ angew Geol 28: 366-370

Pertold Z, Pertoldová J, Potužák M, Pudilová M, Bendl J, Janoušek V, Zachariáš J, Spiro B, Greenwood PB (1997) Skarns of the Bohemian Massif: origin in closed or open systems? J Czech Geol Soc 42: 69

Pertold Z, Pertoldová J, Pudilová M (2000) Metamorphic history of skarns in the Gföhl Unit, Moldanubicum, Bohemian Massif, and implication for their origin. Acta Univ Carol, Geol 41: 157-166

Pertoldová J (1986) Conditions of Genesis of Skarns at Pernštejn, Županovice and Nové Město pod Smrkem Deposits. Unpublished $\mathrm{PhD}$ thesis, Charles University, Prague, pp 1-86 (in Czech)

Pertoldová J, Pertold Z, Pudilová M (1998) Metamorphic development of skarns at Pernštejn, Svratka Crystal- 
line Complex, Bohemian Massif. J Czech Geol Soc 43: 191-202

Pertoldová J, Buriánek D, Hrdličková K, Č́apek D, Čopjaková R, Schulmannová B, Dušek K, Haloda J, Hanžl P, Konopásek J, KopaČKová V, KoŠuličová M, Malec J, Nahodilová R, Soejono I, Sulovský P, ŠKoda R, ŠTĚdrá V, TÁborský Z, Tajčmanová L, TÝCOVÁ P, Verner K, Veselovský F, Vondrovic L, VRÁnA S, Zavřelová A, ŽáčKová E, ŠrÁmek J, Melichar R (2007) Investigation of lithologically contrasting rocks in the crystalline units at the NE margin of the Moldanubian Zone - continuation of basic geological mapping $1: 25$ 000. Unpublished report, Czech Geological Survey, Prague, pp 1-333 (in Czech)

Pitra P, Guiraud M (1996) Probable anticlockwise P-T evolution in extending crust: Hlinsko region, Bohemian Massif. J Metamorph Geol 14: 49-60

PотUŽÁk M (1996) Skarn in Vlastějovice at Holý vrch, Unpublished MSc. thesis, Charles University, Prague, pp 1-138 (in Czech)

Powell R (2005) THERMOCALC, (v. tc325), http://www. earthsci.unimelb.edu.au/tpg/thermocalc

Powell R, Holland T (2004) Course notes for "THERMOCALC workshop 2004: calculating metamorphic phase equilibria" (ETH Zurich). (CD-ROM)

Powell R, Holland T, Worley B (1998) Calculating phase diagrams involving solid solutions via non-linear equations, with examples using THERMOCALC. J Metamorph Geol 16: 577-588

Rejchrt M, Břízová E, Fürych V, Hanžl P, Hradecká L, HrdličKová K, Kadlecová R, Kirchner K, Lysenko V, Mlčoch B, Nahodilová R, Novák M, Otava J, Pertoldová J, Rambousek P, Roštínský P, Rudolský J, SkÁcelová D, SkÁcelová Z, TýcovÁ P, Vít J, ŽáčKovÁ E (2009) Basic geological map ČR 1:25 000 with explanations, 23-222 Krucemburk. Czech Geological Survey, Prague, pp 1-90

RichARD LR (1995) MinPet: Mineralogical and petrological data processing system, version 2.02. MinPet Geological Software, Québec, Canada

RICHARD LR, CLARKE DB (1990) AMPHIBOL: a program for calculating structural formulae and for classifying and plotting analyses of amphiboles. Amer Miner 75: 421-423

Rickwood PC (1968) On recasting analyses of garnet into end member molecules. Contrib Mineral Petrol 18: 175-198

Robinson P, Spear FS, Schumacher JC, Laird J, Klein C, Evans BW, Doolan BL (1981) Phase relations of metamorphic amphiboles: natural occurrence and theory. In: Veblen DR, Ribbe PH (eds) Amphiboles: Petrology and Experimental Phase Relations. Mineralogical Society of America Reviews in Mineralogy 9B: 1-228

Rötzler K, Mingram B (1998) Evolution and geotectonic significance of high-pressure metarodingites in the Erzgebirge; Germany. Acta Univ Carol, Geol 42: 326-327
Scharbert S, Batík P (1985) The age of the Thaya (Dyje) Pluton. Verh Geol Budesanst (Wien) 3: 325-331

Schulmann K, Kröner A, Hegner E, Wendt I, Konopásek J, LEXA O, ŠTíPSKÁ P (2005) Chronological constraints on the pre-orogenic history, burial and exhumation of deepseated rocks along the eastern margin of the Variscan Orogen, Bohemian Massif, Czech Republic. Amer J Sci 305: 407-448

Schulmann K, Lexa O, Štípská P, Racek M, TajČmanová L, Konopásek J, Edel JB, Peschler A, Lehmann J (2008) Vertical extrusion and horizontal channel flow of orogenic lower crust: key exhumation mechanisms in large hot orogens? J Metamorph Geol 26: 273-297

Schulmann K, Konopásek J, Janoušek V, Lexa O, Lardeaux JM, Edel JB, ŠTíPskÁ P, Ulrich S (2009) An Andean type Palaeozoic convergence in the Bohemian Massif. C R Geosci 341: 266-286

Sláma J, Košler J, Condon DJ, Crowley Jl, Gerdes A, Hanchar JM, Horstwood MSA, Morris GA, Nasdala L, Norberg N, Schaltegger U, Schoene B, Tubrett MN, Whitehouse MJ (2008) Plešovice zircon - a new natural reference material for $\mathrm{U}-\mathrm{Pb}$ and $\mathrm{Hf}$ isotopic microanalysis. Chem Geol 249: 1-35

Synek J, OliveriovÁ D (1993) Terrane character of the northeast margin of the Moldanubian Zone: the Kutná Hora Crystalline Complex, Bohemian Massif. Geol Rundsch 82: 566-582

Šrein V, Š́reinOVÁ B (2000) Mineralogy of the skarns of the Bohemian part of the western and central Krušné hory mountains. Acta Montana - series A17: 67-108

ŠTĚDRÁ V, NAHODILOVÁ R (2009) High-pressure metabasic rocks from the Kutná Hora Complex: geological position and petrology of exotic lithologies along the segmented Moldanubian margin, Bohemian Massif. J Geosci 54: 135-157

ŠTíPSKÁ P, Pitra P, Powell R (2006) Separate or shared metamorphic histories of eclogites and surrounding rocks? An example from the Bohemian Massif. J Metamorph Geol 24: 219-240

TAit J, Schätz M, Bachtadse V, Soffel H (2000) Paleomagnetism and Paleozoic paleogeography of Gondwana and European terranes. In: Franke W, HaAk V, Oncken O, TANNER D (eds) Orogenic Processes: Quantification and Modeling in the Variscan Belt. Geol Soc London Spec Publ 179: 21-34

TajČmanová L, KonopáseK J, Schulmann K (2006) Thermal evolution of the orogenic lower crust during exhumation within a thickened Moldanubian root of the Variscan belt of Central Europe. J Metamorph Geol 24:119-134

TAYLOR HP (1979) Oxygen and hydrogen isotope relationships in hydrothermal mineral deposits. In: BARNES HL (ed) Geochemistry of Hydrothermal Ore Deposits. John Wiley \& Sons, New York, pp 236-277

TAYlor SR, McLennan SM (1985) The Continental Crust: 
Its Composition and Evolution. Blackwell, Oxford, pp $1-312$

Tichomirowa M, Berger HJ, Koch EA, Belyatski BV, Götze J, Kempe U, Nasdala L, Schaltegger U (2001) Zircon ages of high-grade gneisses in the Eastern Erzgebirge (Central European Variscides) - constraints on origin of the rocks and Precambrian to Ordovician magmatic events in the Variscan foldbelt. Lithos 56: 303-332

Urban M, Synek J (1995) Moldanubian Zone; Structure. In: Dallmeyer RD, Franke W, Weber K (eds) Pre-Permian Geology of Central and Eastern Europe. Springer Verlag, Berlin, pp 429-443

Van Breemen O, Aftalion M, Bowes DR, Dudek A, Mísař Z, PovONDRA P, VRÁNA S (1982) Geochronological studies of the Bohemian Massif, Czechoslovakia, and their significance in the evolution of Central Europe. Trans Roy Soc Edinb: Earth Sci 73: 89-108

Verner K, Buriánek D, Vondrovic L, Pertoldová J, NAHOdilová R, HANŽL P (2009) Tectonometamorphic features of geological units along the northern periphery of the Moldanubian Zone (Bohemian Massif). J Geosci 54: $87-100$

VRÁNA S (1987) Garnet-fassaitic pyroxene skarn from the granulite complex of southern Bohemia. Věst ústř Úst geol 62: 193-206

VRÁNA S, KRÖNER A (1995) Pb-Pb zircon ages for tourmaline alkali-feldspar orthogneiss from Hluboká nad Vltavou in southern Bohemia. J Czech Geol Soc 40: 127-131

VRÁNA S, ŠTĚdRÁ V, FišERA M (2005) Petrology and geochemistry of the Běstvina granulite body metamorphosed at eclogite facies conditions, Bohemian Massif. J Czech Geol Soc 50: 95-106
VRÁNA S, ŠTĚDRÁ V, Nahodilová R (2009) Geochemistry and petrology of high-pressure kyanite-garnet-albite$\mathrm{K}$-feldspar felsic gneisses and granulites from the Kutná Hora Complex, Bohemian Massif. J Geosci 54: 159-179

White RW, Powell R, Holland TJB (2001) Calculation of partial melting equilibria in the system $\mathrm{Na}_{2} \mathrm{O}-\mathrm{CaO}-$ $\mathrm{K}_{2} \mathrm{O}-\mathrm{FeO}-\mathrm{MgO}-\mathrm{Al}_{2} \mathrm{O}_{3}-\mathrm{SiO}_{2}-\mathrm{H}_{2} \mathrm{O}$ (NCKFMASH). J Metamorph Geol 19: 139-153

Wiedenbeck M, Alle M., Corfu F, Griffin WL, Meier M, Oberli F, von Quadt A, Roddick JC, Spiegel W (1995) Three natural zircon standards for U-Th- $\mathrm{Pb}, \mathrm{Lu}-\mathrm{Hf}$, trace element and REE analyses. Geostand Newsl 19: 1-23

Zheng ZG, Wang XY, Zhang GL, Yin XB, Chen DG, WAng XM (2008) Formation of Fe-oxyhydroxides from the East Pacific Rise near latitude $13^{\circ} \mathrm{N}$. Evidence from mineralogical and geochemical data. Sci China Ser D Earth Sci 51: 206-215.

ZoubeK V (1946) Notes on the geology of the crystalline complexes of the Bohemian Massif, II. Sbor Stát Geol Úst 25: 339-398.

Ž́́ČEK V (1997) Compositional evolution of garnet in the regionally metamorphosed Moldanubian skarn, Vlastějovice, Bohemia - evidence of the preservation of early stages pre-dating regional metamorphism. Bull Czech Geol Surv 72: 37-48

Žáčé V, Novák M, Raimboult L, Zachariáš J, Ackerman L (2003) Locality No 8, Vlastějovice near Ledeč nad Sázavou. In: NovÁK M (ed) - LERM 2003, International Symposium on Light Elements in Rock-forming Minerals, Field Trip Guidebook, Masaryk University Brno, pp 61-70. ISSN 80-7028-198-7 